\title{
APPROXIMATE SOLUTIONS FOR COMPRESSIBLE TURBULENT \\ BOUNDARY LAYERS IN THREE-DIMENSIONAL FLOW
}

\author{
A THESIS \\ Presented to \\ The Faculty of the Graduate Division \\ by \\ Richard Gordon Bradley, Jr. \\ In Partial Fulfillment \\ of the Requirements for the Degree \\ Doctor of Philosophy in the \\ School of Aerospace Engineering
}

Georgia Institute of Technology

June, 1966 
In presenting the dissertation as a partial fulfillment of the requirements for an advanced degree from the Georgia Institute of Technology, I agree that the Library of the Institute shall make it available for inspection and circulation in accordance with its regulations governing materlals of this type. I agree that permission to copy from, or to publish from, this dissertation may be granted by the professor under whose direction it was written, or, In his absence, by the Dean of the Graduate Division when such copying or publication is solely for scholarly purposes and does not involve potential financial gain. It is understood that any copying from, or publication of, this dissertation which involves potential financial gail will not be allowed without written permission.

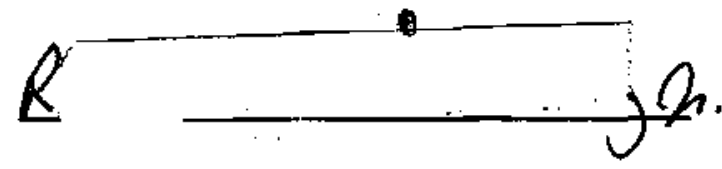

$3 / 17 / 65$

b 
APPROXIMATE SOLUTIONS FOR COMPRESSIBLE IURBULENT

BOUNDARY LAYERS IN THREE-DIMENSIONAL FLOW

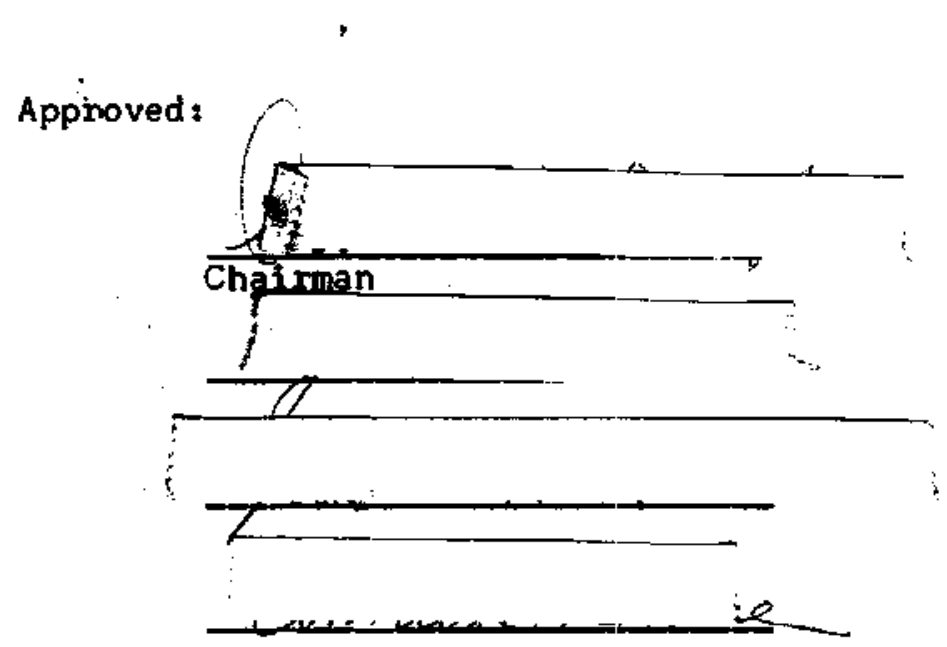

Date approved by Chairman: $6 / 2 / 66$

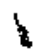




\section{ACKNONLEDGMENTS}

The author wishes to express his appreciation to his thesis advisor, Dr. A. L. Ducoffe, Director of the School of Aerospace Engineering, for his interest, assistance, and encouragement throughout the course of this research. The author is also indebted to Professors J. E.

Hubbartt, J. C. Wu, and A. C. Bruce for many helpful discussions as well as for their careful review of the work.

Appreciation is expressed to General Dynamics, Fort Worth

Division, whose financial assistance, not only during the course of the research but throughout the author's graduate studies, made this work possible.

The author is grateful to his wife whose patience and understanding contributed greatly to the completion of the research. 
TABLE OF CONTENTS

Page

ACKNOWLEDGMENTS ........................ . . i i

LIST OF ILLUSTRATIONS .................... . . v

NOMENCLATURE . . . . . . . . . . . . . . . . . . . . vii

SUMMARY ............................ x

Chapter

I. INTRODUCTION .................... 1

Historical Sketch

Purpose of the Study

II. THEORETICAL FORMULATION . . . . . . . . . . . 10

Coordinate System and Governing Equations

Small Cross-Flow

Integral Equations

Solution Considerations

III. METHOD OF SOLUTION . . . . . . . . . . . . 22

Streamwise Flow

Cross-Flow

Summary

IV. RESULTS FOR A YAWED INFINITE CYLINDER . . . . . . 38

Inviscid Flow and Geometric Relations

Stagnation Line Solutions

Streamwise Flow Solutions

Cross-Flow Solutions

V. CONCLUSIONS AND RECOMMENDATIONS . . . . . . . . . 80 Appendices

A. BOUNDARY LAYER INTEGRAL EQUATIONS ............. 85

B. TRANSFORMATION OF THE CROSS-FLOW MOMENTUM EQUATION $\ldots . . \quad 89$ 
TABLE OF CONTENTS (Continued)

Page

Appendices

C. THE CROSS-FLOW VELOCITY PROFILE AND RESULTING MOMENTUM INTEGRAL EQUATION ............. 93

D. GEOMETRIC PROPERTIES FOR A YAWED INFINITE CYLINDER ............................. 98

E. THE Reference temperature . . . . . . . . 103

F. RELATION BETWEEN THE COMPRESSIBLE AND TRANSFORMED velocitr Profiles . . . . . . . . . . 109

G. MAXIMUM CROSS-FLOW VELOCITY ................. 112

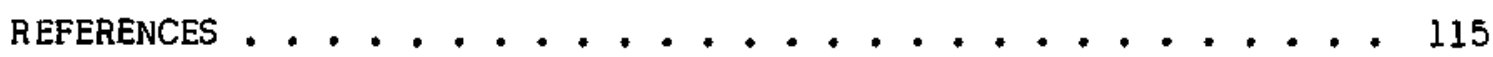

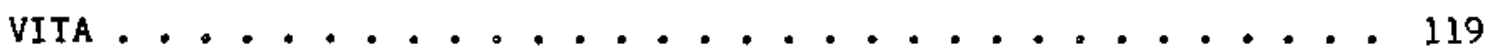




\section{LIST OF ILLUSTRATIONS}

Figure

Page

1. The Streamline Coordinate System . . . . . . . . . 11

2. Surface Coordinates on a Yawed Infinite Cylinder . . . . 40

3. Local Mach Number Distribution for a Yawed Infinite Cylinder ...................

4. Distance Along the Streamline Coordinate for a Yawed Infinite Cylinder .............. 4 46

5. Streamline Divergence for a Yawed Infinite Cylinder . . . 47

6. Inviscid Streamline Curvature Parameter for a Yawed Infinite Cylinder ...............

7. Computed Boundary Layer Parameters on the Stagnation Line ..................

8. Stagnation Line Heat Iransfer - Comparison with Experiment, $M_{\infty}=4.15$...............

9. Stagnation Line Heat Transfer as a Function of Yaw Angle, $M_{\infty}=4.15$............... 54

10. Computed Variation of $\mathrm{H}_{\mathbf{i}}$ for the Yawed Cylinder . . . 56

11. Computed Momentum Thickness for the Yawed Cylinder . . . 57

12. Computed Displacement Thickness for the Yawed Cylinder .........................

13. Computed Streamwise Skin Friction Coefficient for the Yawed Cylinder ...................

14. Comparison of Computed Heat Transfer with Experiment for the Yawed Cylinder .................

15. Cross-Flow Solutions - Yawed Cylinder Example .....

16. Maximum Value of Cross-Flow to Streamise Velocity Ratio .......................

17. Ratio of Cross-Flow to Streamwise Skin Friction Components...................... 
18. Typical Velocity Profiles for the Yawed Cylinder Example, $\Lambda=60^{\circ}$................ 68

19. Maximum Cross-Flow Velocity for the Yawed Cylinder . . . 69

20. Typical Velocity Profiles for Three Assumed Shape Relations, $\Lambda=60^{\circ}$............. 72

21. Effect of Velocity Profile Assumptions on the CrossFlow Solution, $\Lambda=10^{\circ}$............ 73

22. Effect of Velocity Profile Assumption on the CrossFlow Solution, $\Lambda=60^{\circ} \ldots \ldots . . . \ldots$

23. Effect of Cooling on the Cross-Flow Solution,

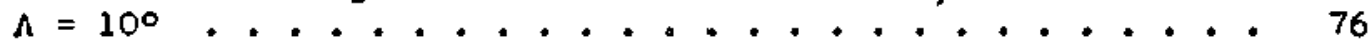

24. Effect of Cooling on the Cross-Flow Solution, $\Lambda=60^{\circ}$...................... 77

25. Comparison with Solution Holding $\mathrm{H}_{i}$ Constant,

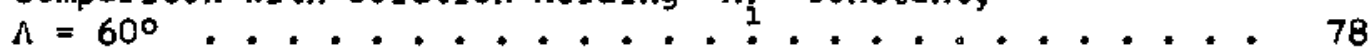

E-1. Insulated Flat Plate Example . . . . . . . . . 105

E-2. Mach Number Distribution for Favorable Pressure Gradient Example . . . . . . . . . . . 107

E-3. Favorable Pressure Gradient Example . . . . . . . . 108

G-1. Effect of $H_{j}$ on Maximum Cross-Flow Velocity for Three Profile Assumptions ................... 114 
NOMENCLATURE

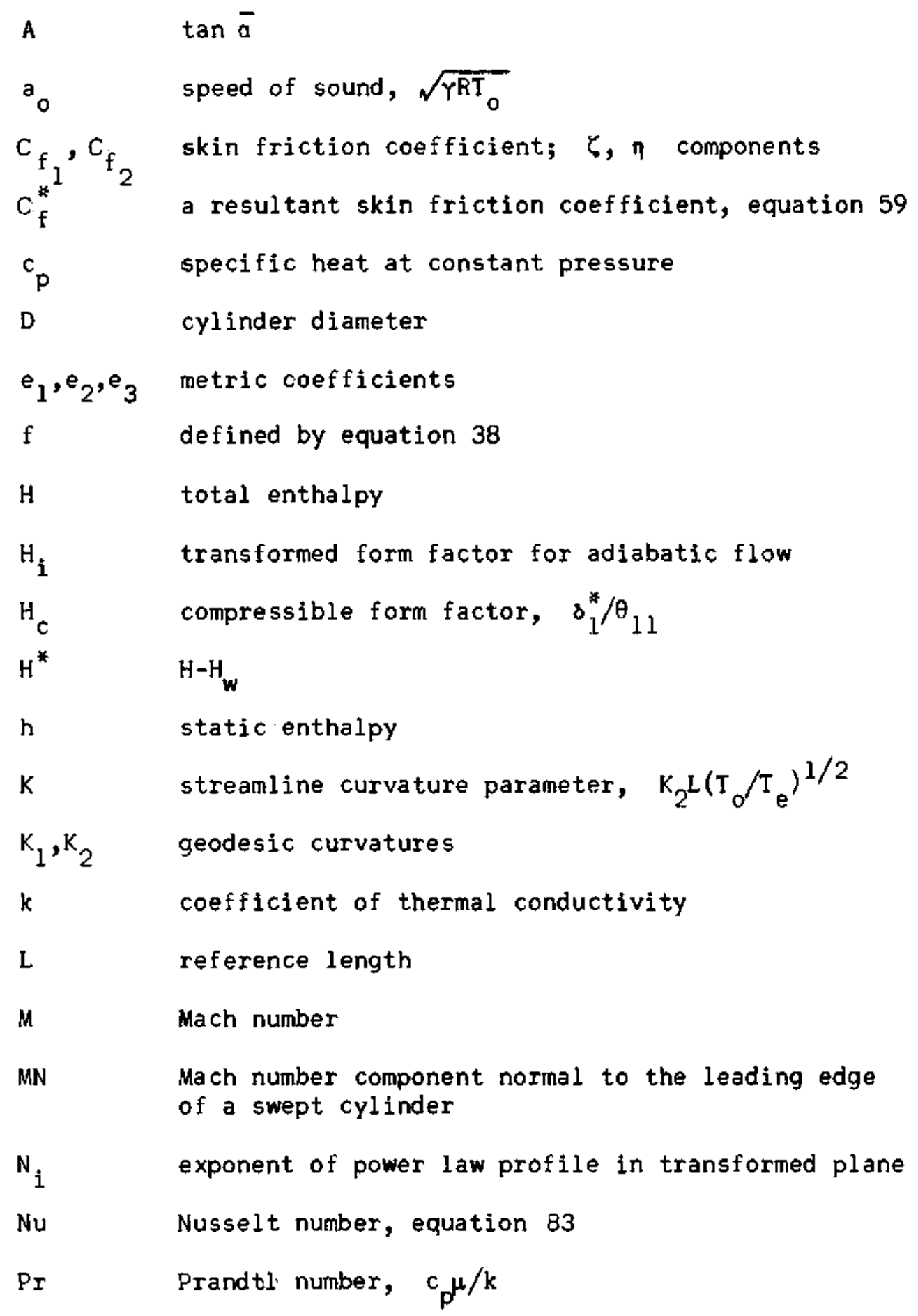




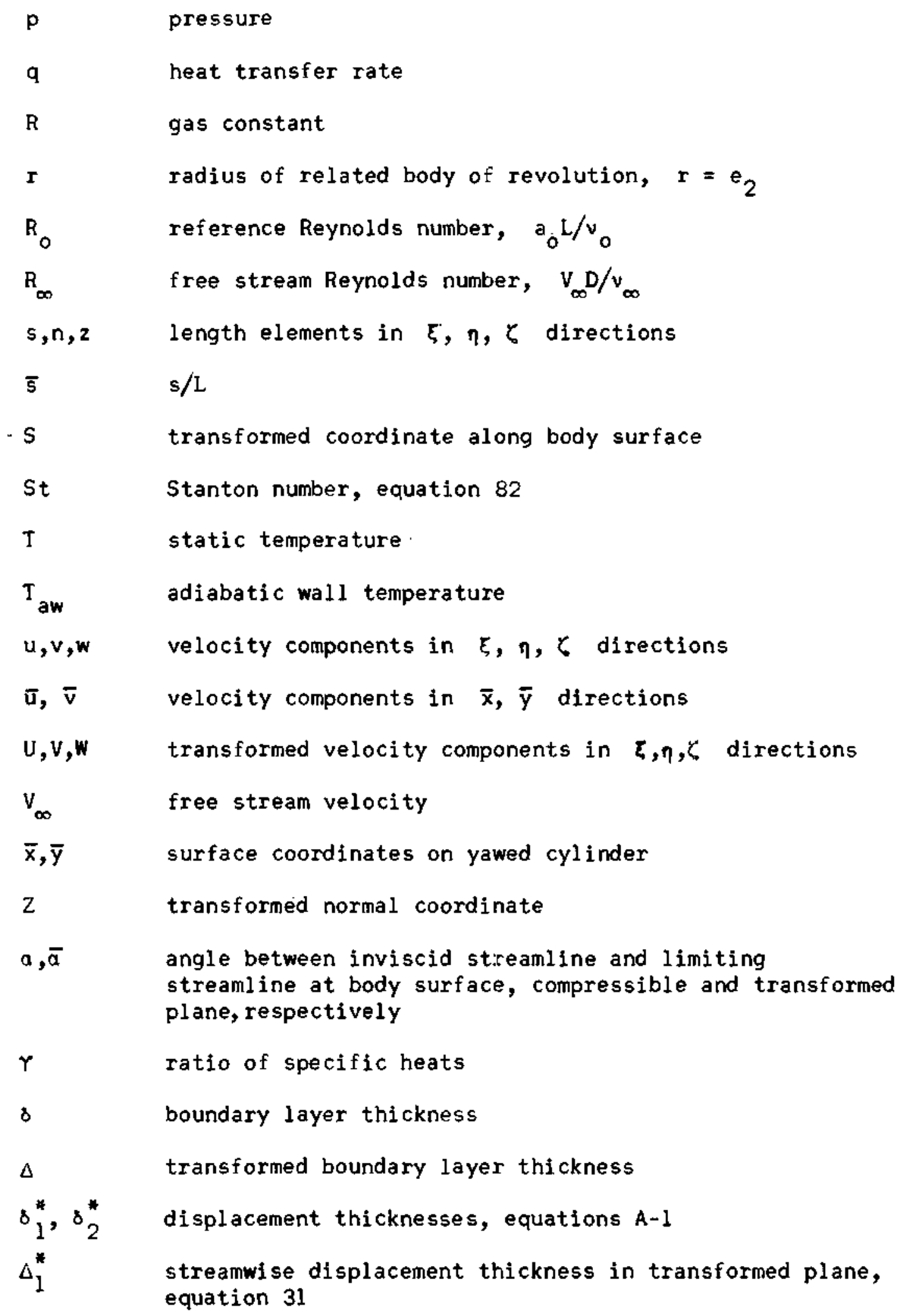




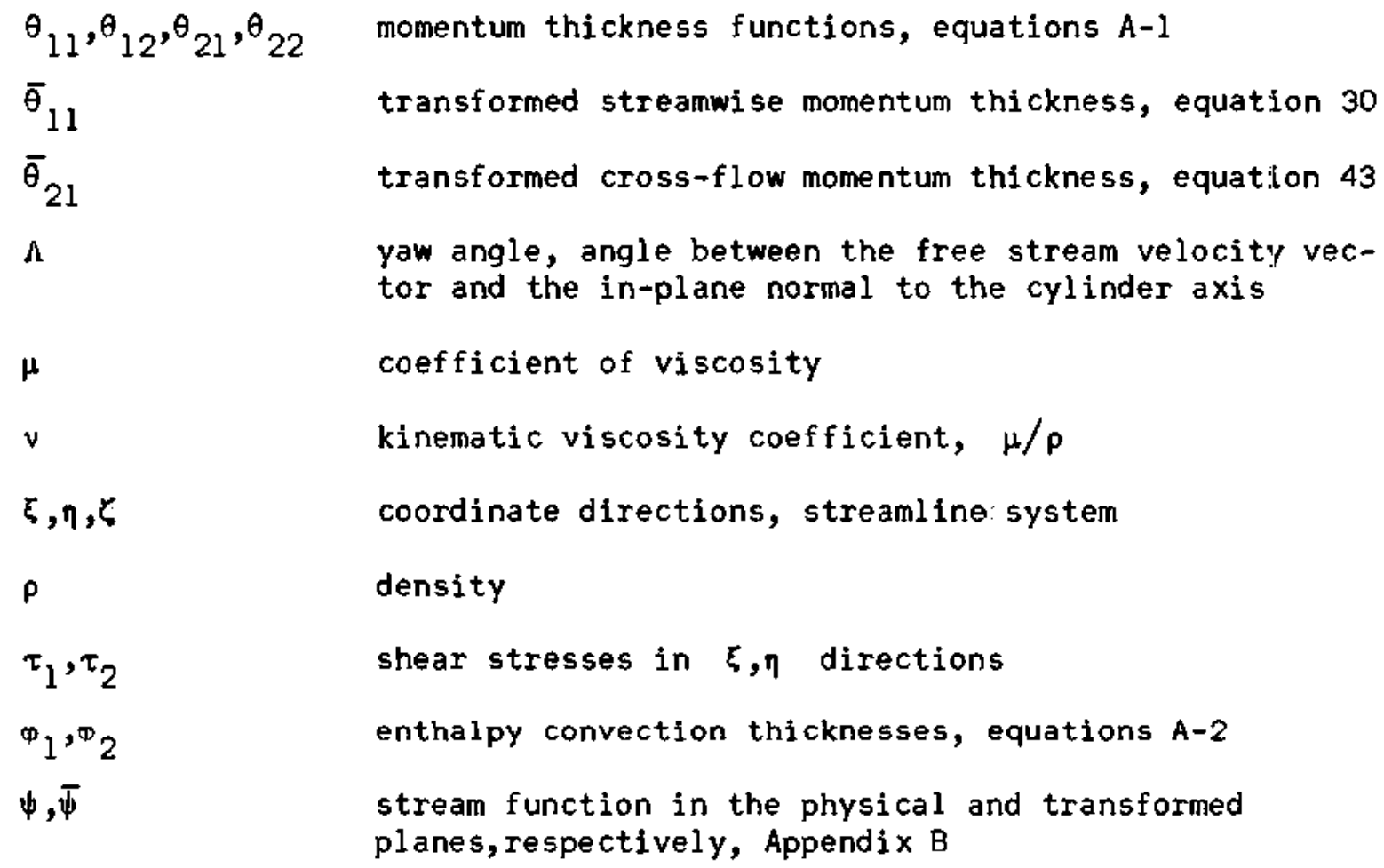

transformed streamwise momentum thickness, equation 30 transformed cross-flow momentum thickness, equation 43 yaw angle, angle between the free stream velocity vector and the in-plane normal to the cylinder axis

coefficient of viscosity

kinematic viscosity coefficient, $\mu / \rho$

coordinate directions, streamline: system

density

shear stresses in $\xi, \eta$ directions

enthalpy convection thicknesses, equations A-2

stream function in the physical and transformed planes, respectively, Appendix B

\section{Subscripts}

e

$\circ$

\section{$r$}

sl

w

$\infty$

\section{Superscript}

conditions at boundary layer outer edge

local inviscid flow stagnation conditions

evaluated at reference conditions

stagnation line conditions, yawed cylinder

conditions at body surface

free stream conditions

fluctuating component 


\section{SUMMARY}

The compressible turbulent boundary layer equations for an arbitrary three-dimensional surface are simplified by assuming that the component of flow within the boundary layer normal to the inviscid streamline direction, called cross-flow or secondary flow, is small. The equations of continuity, momentum, and energy are first written in a streamline coordinate system where the trace of the inviscid flow streamline upon the surface forms one family of coordinates and the orthogonal trajectories of these curves define a second family. The assumption of small secondary flow in this coordinate system reduces the governing equations along a particular inviscid streamline to the form taken for flow over an axially symmetric body where the local radius of the equivalent body is given by a metric coefficient of the coordinate system. This simplification allows solution of the equations describing the strearrise flow, that is, the flow along the path of an inviscid streamline independent of the cross flow. Solution of the cross-flow momentum equation is then possible subject to the streamwise solution. The integral equations of energy, streamwise momentum, and cross-flow momentum are derived assuming small secondary flow. A computation procedure for approximate solution of these equations is presented.

Full advantage of the analogy between the streamwise flow and a related axially symmetric one is taken in that a computation method of proven utility for axisymmetric flows is applied directly to the three- 
dimensional boundary layer with small cross-flow. The computation procedure for the streamwise flow equations is based on a method given by Sasman and Cresci (AIAA J., 4, 19, 1966). This method simplifies the streamwi se momentum and corresponding moment-of-momentum integral equations by means of a Mager-type transformation. Upon specification of an appropriate skin friction law and assuming the Crocco velocity-temperature relation holds, simultaneous integration of these coupled differential equations provides boundary layer thickness functions, skin friction, and related quantities.

The cross-flow momentum equation is simplified by the same trans: formation employed in the streamise solution. After assuming an appropriate form for the cross-flow velocity profile and specifying a curvature parameter as determined by the streamline coordinate system, the resulting integral equation is integrated along an inviscid streamline using the results of the streamwise computations. The assumed cross-flow velocity profile for this calculation is based on limited experimental evidence for incompressible and compressible turbulent boundary layers. This solution provides the angle between the inviscid flow direction and the direction of the velocity vector as the surface is approached. The value of the cross-flow component of skin friction and an indication of the limits of applicability of the small cross-flow assumption are then determined.

The computation procedure is applied to a yawed infinite cylinder example. The streamline coordinate system and required curvature parameters are defined consistent with the cylinder geometry and the inviscid flow. Solutions for both the streamise flow and the cross-flow are 
presented for yaw angles of $10,20,40$, and 60 degrees with a free stream Mach number equal to 4.15 . Heat transfer computed from the streamwise solutions assuming a form of Reynolds analogy strictly valid for flat plate flow is compared with available experimental data on the stagnation line and over the forward face of the cylinder. Along the stagnation line the theoretical heat transfer is found to be in good agreement with experiment for all yaw angles. Away from the stagnation line good agreement is also noted for yaw angles of 40 and 60 degrees, but the theory gives predictions as much as 80 percent above the experimental data for the 10 and 20 degree yaw angles. The use of Reynolds analogy is considered questionable for these low yaw cases because of the la:rge streamwise pressure gradient present.

Solutions of the cross-flow momentum integral equation for the yawed cylinder example reveal that the cross-flow is not large for the four yaw angles considered. The maximum secondary flow occurs for the 60 degree yaw case. The good agreement with the experimental heat transfer data afforded by the streamwise solution for this case verifies that the cross-flow effect is small for the flow conditions considered.

In view of the existing uncertainty in the exact shape of the cross-flow velocity profile for general flow conditions, solutions to the cross-flow momentum integral equation are compared for three assumed profile relations. The results show that the solution is dependent on the profile assumption to a significant degree thus indicating a need for experimental studies to establish the correct form for different flow conditions.

Increasing the cooling at the surface is shown to reduce the cross-flow magnitude as is expected from physical arguments. As a result 
the small cross-flow assumption may be generally more useful for problems with a high degree of cooling.

The cross-flow solution is shown to be dependent to a significant degree on the streamwise velocity profile so that changes in this profile resulting from pressure gradient have a pronounced effect on the secondary flow solution. Thus, when calculation of the cross-flow is desired it is important that the technique for computing the streamwise flow take into account the velocity profile development. 


\section{CHAPTER I}

\section{INTRODUCT ION}

The calculation of the properties of a turbulent boundary layer under general flow conditions is of recognized importance in a great many engineering applications. In the development of aerospace vehicles, for example, computation methods for predicting skin friction, boundary layer thickness functions, and heat transfer on surfaces of general anti-symmetric shape, bodies at angle of yaw, swept wings, etc. are of primary concern. The research reported in the following chapters is motivated by the existing need for adequate prediction of the engineering parameters associated with turbulent boundary layers in compressible, three-dimensional flow.

The study of turbulent boundary layers is generally acknowledged as a task of some complexity in that the random, fluctuating motion of the fluid is not amenable to satisfactory theoretical treatment. Further, such physical phenomena as shear stress and heat transfer are proportional to the turbulent transport properties and no longer are related to parameters which are a function of the fluid alone. Beyond these fundamental considerations, additional complexities such as compressibility of the fluid, heat transfer to the surface, three dimensionality of the flow, and the presence of pressure gradients are realities of practical flows which cannot be ignored in many instances.

Although the problems associated with turbulent flow have long been the object of investigation, as evidenced by the vast amount of 
literature available, progress in precise description of the flow has been slow. Generally two avenues of approach have become manifest in the literature:

(a) attempts to define a mathematical model based for the most part on statistical methods, and

(b) empirical formulation which relies heavily on parameters determined from experiment.

From a purely scientific point of view, the former approach stands as requisite to accurate calculation of the turbulent boundary layer. However, even though significant progress has been realized, development to the point that engineering calculations can be made has yet to be achieved. On the other hand, if one takes a pragmatic point o: view the latter approach of fers a line of attack for current problems which, though admittedly limited in scope by the amount of experimental data available, provides solutions of quantitative value in many engineering applications. It is within this second line of thought that the work reported herein is founded.

The problem to be considered is the approximate computation of the turbulent boundary layer parameters for compressible, three-dimensional flow. By way of introduction a brief historical sketch is presented including a short summary of several papers in the literature which treat the compressible problem directly. The objectives of the current study are then delineated.

\section{Historical Sketch}

The subject of three-dimensional boundary layers has been reviewed 
by several authors in the past two decades. Reviews by Sears (1) in 1954 and Moore (2) in 1956 deal primarily with laminar flows. Cooke and Hall (3) in 1962 presented an extensive survey of both laminar and turbulent work. Cooke (4) in 1963 reviewed the turbulent literature exclusively. The above works present collectively a comprehensive list of references dealing with three-dimensional boundary layers, the overwhelming majority of which concern laminar flow. Laminar boundary layers have been studied quite broadly from the standpoint of exact numerical treatments as well as series expansions and approximate integral methods (e.g. see references $1 \div 3,5,6$, and 7$)$.

In contrast to the laminar boundary layer the turbulent layer has been largely neglected in the literature. Cooke (4) attributes this neglect to the difficulty of the subject and to the fact that the collection of experimental data is "tedious and unrewarding." In the work that has been done momentum integral methods have been used exclusively and two-dimensional methods have formed the basis for needed empirical laws of skin friction and velocity profiles.

Two concepts have proven extremely convenient in laminary boundary layer analysis and have been fundamental to almost all the turbujent work. These are the use of streamline coordinates and an analogy relating certain three-dimensional flows to an axially symmetric one. The streamline coordinate system is one in which one family of coordinate curves is the projection of the external streamlines onto the surface of the body while the other family consists of the orthogonal trajectory of the first family. Flow in the inviscid streamline direction is termed streamwise flow whereas flow normal to this direction is called cross-flow 
(or sometimes secondary flow). In the event that the cross-flow is small the equations of motion in the streamwise direction are identical in form to those for an axially symmetric boundary layer flow. Further, the streamwise equations are no longer coupled with the cross-flow momentum equation. Eichelbrenner and Oudart (B) first noted this fact for incompressible flow and Cooke (9) has pointed out that the same is true for compressible flow.

Computation methods have been developed by Mager (10), Cooke (11), Becker (12), and Zaat (13) for incompressible turbulent flows. These methods differ basically from one another in the assumptions concerring velocity profiles and friction laws and are reviewed in reference (4). Only a rather meager body of experimental data is available for formulation of these calculation techniques. The experimental work of Gruschwitz (14), Kuethe, McKee and Curry (15), Johnston (16), Wallace (17), Brebner and Wyatt (18), and Blackman and Joubert (19), all incompressible, serve in the main as justification for the necessary computational approximations.

The need for careful experimentation is perhaps best illustrated by the fact that a review of the literature revealed only one paper reporting detailed measurements within a compressible, three-dimensional, turbulent boundary layer. This work reported recently by Hall (24) deals with the flow over the sidewall of a specially constructed supersonic nozzle. Profiles of streamwise and cross-flow velocity are presented along with measurements of local skin friction.

Four papers dealing directly with the computation of compressible, turbulent boundary layers were found in the avajlable literature. Each 
of these is reviewed briefly here in order to form a frame of reference for the presentation to follow.

Braun (20)

Braun considered the particular case of a cone at small angle of yaw. For this case he derived the two momentum integral equations in streamline coordinates and applied two-dimensional methods in the streamwise direction, after implicitly assuming small cross-flow. Braun showed that the pressure variation along an inviscid streamline was negligible when the yaw angle was small, thus permitting the use of a $\mathrm{Bl}$ asius flat plate friction law corrected to the compressible flow by the reference temperature method, a power velocity profile in the streamwise direction, and the flat plate Crocco relation between temperature and velocity profiles. A form for the cross-flow velocity profile suggested by low speed experiments of Gruschwitz (14) was assumed.

With these approximations Braun found solutions to the momentum integral equations by expanding the boundary layer thickness, 8, and the angle between the inviscid streamline direction and the limiting direction of the velocity vector as the surface is approached, a, in terms of a yaw parameter for the cone at small incidence. Local skin friction coefficient, lift coefficient, pitching moment coefficient, and a were presented for several supersonic Mach numbers and for three wall to free stream temperature ratios.

Vaglio-Laurin (21)

Vaglio-Laurin considered the turbulent boundary layer for 
flow conditions typical of cooled blunt bodies in three-dimensional hypersonic flow, i.e. moderate local Mach number with high surface cooling. Under these conditions it was shown that the cross-flow velocity approaches zero. Thus Vaglio-Laurin treated the streamwise flow in a streamline coordinate system using two-dimensional methods and discarded the cross-flow momentum equation.

A transformation to an equivalent incompressible flow given by Mager (25) was employed. The effect of pressure gradient was shown to be negligible for this particular class of flows, and a solution to the momentum equation was found using a Von Karman logarithmic velocity law in the transformed plane along with the assumption of a solution of the energy equation in accord with Reynolds analogy.

A heat transfer expression was derived using these results. Application of the method was given for some axisymmetric flow examples in the absence of experimental data for three-dimensional cases. Beckwith and Gallagher (22)

Beckwith and Gallagher developed a computation method for a yawed infinite cylinder. The integral equations of momentum and energy were written in a coordinate system defined by surface distances in the chordwise and spanwise directions. A modified Stewartson coordinate transformation was applied in order to set: the problem in an incompressible plane. Power velocity profiles were assumed in the chordwise and spanwise directions. Solutions were obtained by assuming a Blasius type skin 
friction law to hold in the local resultant inviscid flow direction (streamwise in a streamline coordinate frame of reference). Zero secondary flow was postulated throughout. An expression for heat transfer was developed from the analysis by applying the flat plate Reynolds analogy relating the heat transfer and resultant shear.

Experimental heat transfer data were obtained by Beckwith and Gallagher (22) for a yawed cylinder at Mach number of 4.15 and high Reynolds numbers. The theory was compared with theseidata. Cooke (4)

Cooke has suggested a calculation method of a somewhat more general nature than any of those discussed previously. The technique is an extension of his earlier work for incompressible flow (11) to the compressible case. The governingl momentum integral equations are written in streamline coordinates and small crossflow is assumed. Making use of the axisymmetric analogy, a twodimensional method developed by Spence (23) is employed to solve the streamwise momentum integral equation.

The Spence method features a compressibility correlation of the Stewartson type, a quadratic velocity-temperature relation, and a power law skin friction expression related to the compressible flow by the reference temperature concept. The solutions thus obtained are applicable to insulated walls or isothermal cooled walls with streamwise pressure gradient.

A specialization of this method to the case of swept infinite wings has been given in reference 26 . Here the streamwise 
equations are derived, but no calculations are presented. In addition, application of the method has been reported (27) for a thin delta wing at zero lift and a Mach number of two. For this case the three-dimensional effects were found to be small.

At this point several observations concerning the computation methods just discussed are appropriate. In each instance small (or zero) crossflow was assumed, either explicitly or implicitly, thus allowing experience in solving two-dimensional turbulent boundary layers to serve as a basis for the solution of the three-dimensional flow.

Of the methods summarized only Braun found a solution for the crossflow momentum equation, which was for the slightly yawed cone example. In the event that the cross-flow is in fact vanishingly small such a solution is of little interest so far as the determination of the boundary layer parameters is concerned. However, solutions to the cross-flow momentum equation can be of significant value as an indicator of when the small secondary flow assumption may be in error for a particular problem.

Three of the four solution techniques have been formulated for application to a particular problem with certain of the assumptions being justified accordingly. Only the method of Cooke may be considered general in that no specific geometry or flow conditions are postulated (beyond the small cross-flow assumption and isothermal or adiabatic wall restriction). In Cooke's method the effect of streamise pressure gradient has been taken into account in the momentum equation. In all cases, however, flat plate skin friction laws were employed and the velocity profiles were assumed to be unaffected by the pressure gradient. 


\section{Purpose of the Study}

The purpose of the research reported in this thesis is to investigate further the features of three-dimensional, compressible, turbulent boundary layers from the standpoint of practical calculation of such flows. The specific objectives of the study are, first, to develop a general method for solving the governing integral equations within the framework of the small secondary flow assumption and, second, to investigate analytically the cross-flow with particular emphasis on the parameters which affect its magnitude.

A method for solving both the streamwise equations and the crossflow equation is developed. An advantage over previous methods stems from the inclusion of pressure gradient effects on the streamwise velocity profiles and the skin friction law. Solutions to the cross-flow momentum equation complement the streamwise solution by indicating the magnitude of the secondary flow, thus giving insight into the probable limitations of the small cross-flow assumption.

In the following chapters, the mathematical model is formulated in terms of an appropriate coordinate system. Then the method of solution is delineated with due regard given to its assumptions and limitations. Finally, a yawed infinite cylinder example is investigated in detail. Solutions are compared with avallable heat transfer data and the effects of several parameters on the cross-flow are studied. 
CHAPTER II

\section{THEORETICAL FORMULATION}

The mathematical specification of the problem is treated in the following paragraphs. The governing boundary layer equations are prosented in a convenient choice of curvilinear, orthogonal coordinates. An important simplification is introduced by assuming that the flow component in a direction normal to the inviscid streamline is small. The integral equations of boundary layer flow are given and the solution of these equations is discussed.

\section{Coordinate System and Governing Equations}

The boundary layer equations are written in a streamline coordinate system as illustrated in Figure 1. These three-dimensional, orthogonal, curvilinear coordinates are specialized to a streamline system intrinsic to the surface and flow under investigation. The $\xi$-curves ( $\eta=$ constant) are taken as the projection of the inviscid streamlines on the body surface and the $\eta$-curves $(\xi=$ constant) are their orthogonal trajectories. The surface is given by $\zeta=0$ and elements of length in the three coordinate directions are giveniby

$$
\begin{aligned}
& d s=e_{1}(\xi, \eta) d \xi \\
& d n=e_{2}(\xi, \eta) d \eta \\
& d z=e_{3} d \zeta
\end{aligned}
$$

where $e_{3}=1$. The above is a simplification of the general metric form 




Figure 1. The Streamline Coordinate System. 
where the metric coefficients $e_{1}, e_{2}$, and $e_{3}$ are functions of $E_{2}$, $\eta$, and $\zeta$. Squire (28) has shown that this approximation is valid to the same order as the Prandtl boundary layer equations provided the surface curvature does not change abruptly.

The velocity components in the coordinate directions $\xi, \eta$, and $\zeta$ are $u, v$, and $w$, respectively. Note that at the outer edge of the boundary layer the velocities $u$ and $v$ become $u=u_{e}$ and $v=v_{e}=0$ in the streamline coordinate system.

The partial differential equations governing the three-dimensional turbulent boundary layer have been derived by Vaglio-Laurin (21). In the streamline coordinate system the equations take the following form.

Continuity:

$$
\frac{\partial}{\partial \zeta}\left(\rho e_{2} u\right)+\frac{\partial}{\partial \eta}\left(\rho e_{1} v\right)+\frac{\partial}{\partial \zeta}\left[\rho e_{1} e_{2}\left(w+\frac{\left(\overline{\rho^{\prime} w^{\prime}}\right)}{\rho}\right)\right]=0
$$

F - Momentum:

$$
\begin{gathered}
\rho\left[\frac{u}{e_{1}} \frac{\partial u}{\partial \xi}+\frac{v}{e_{2}} \frac{\partial u}{\partial \eta}+\left[w+\frac{\left(\overline{p^{\top} w^{\top}}\right)}{\rho}\right] \frac{\partial u}{\partial \zeta}-K_{2} u v+K_{1} v^{2}\right] \\
=-\frac{1}{e_{1}} \frac{\partial \rho}{\partial \xi}+\frac{\partial}{\partial \zeta}\left[\mu \frac{\partial u}{\partial \zeta}-\rho\left(\overline{u^{\top} w^{\top}}\right)\right]
\end{gathered}
$$

$\eta$ - Momentum:

$$
\begin{gathered}
\rho\left[\frac{u}{e_{1}} \frac{\partial v}{\partial \xi}+\frac{v}{e_{2}} \frac{\partial v}{\partial \eta}+\left[w+\frac{\left(\overline{\rho^{\prime} w^{\prime}}\right)}{\rho}\right] \frac{\partial v}{\partial \zeta}-k_{1} u v+k_{2} u^{2}\right] \\
=-\frac{1}{e_{2}} \frac{\partial p}{\partial \eta}+\frac{\partial}{\partial \zeta}\left[\mu \frac{\partial v}{\partial \zeta}-\rho\left(\overline{v^{\prime} w^{\prime}}\right)\right]
\end{gathered}
$$


$\varsigma$ - Momentum:

$$
\frac{\partial p}{\partial K}=0
$$

Energy:

$$
\begin{aligned}
& \rho\left[\frac{u}{e_{1}} \frac{\partial H}{\partial \xi}+\frac{v}{e_{2}} \frac{\partial H}{\partial \eta}+\left[w+\frac{\left(\overline{e^{\prime} w^{\top}}\right)}{\rho}\right] \frac{\partial H}{\partial \zeta}\right] \\
& =\frac{\partial}{\partial \zeta}\left[\mu\left(\frac{\partial H}{\partial \zeta}+\frac{1-\operatorname{Pr}}{\operatorname{Pr}_{r}} \frac{\partial h}{\partial \zeta}\right)-\rho\left(\overline{\bar{w}^{\prime} H^{\top}}\right)\right]
\end{aligned}
$$

In the above $H$ is the total enthalpy given by

$$
H=h+\frac{u^{2}+v^{2}+w^{2}}{2}
$$

and ( ) denotes the time average values of products of the fluctuating components. $K_{1}$ and $K_{2}$ are the geodesic curvatures of the curves $E=$ constant and $\eta=$ constant, respectively, given by

$$
K_{1}=-\frac{1}{e_{1} e_{2}} \frac{\partial e_{2}}{\partial \xi}
$$

and

$$
K_{2}=-\frac{1}{e_{1} e_{2}} \frac{\partial e_{1}}{\partial \eta}
$$

These equations are valid for steady, boundary layer flow of a compressible, perfect gas with constant specifjc heats and constant Prandtl number.

The boundary conditions to be satisfied at all values of $\xi$ and 
$\eta$ are

$$
\begin{aligned}
& \text { Body Surface, } \zeta=0 \\
& \qquad u=v=w=0, H=H_{w},(-)=0 \\
& \text { Outer Edge of Boundary Layer, } \zeta=8 \\
& \qquad u=u_{e}, \quad v=v_{e}=0, H=H_{e},(-)=0
\end{aligned}
$$

where the subscript $w$ refers to values at the wall and the subscript e refers to values at the outer edge of the boundary layer. Evaluation of equations 3 and 4 at the outer edge of the boundary layer results in the following useful relations.

$$
\begin{aligned}
& \frac{\rho_{\mathrm{e}} u_{e}}{e_{1}} \frac{\partial u_{e}}{\partial t}=-\frac{1}{e_{1}} \frac{\partial q}{\partial t} \\
& \rho_{e} u_{e}^{2} K_{2}=-\frac{1}{e_{2}} \frac{\partial p}{\partial \eta}
\end{aligned}
$$

\section{Small Cross-Flow}

Significant simplifications of the boundary layer equations result when the secondary flow is small, as was noted in Chapter I. Here small cross-flow is defined by the requirements that the crosswise components $v$ and $\left(\overline{v^{\prime} w^{\top}}\right)$, and the lateral derivatives $\partial / \partial \eta$ are small. This definition implies physically that the cross-flow velocity and turbulent stress component as well as the gradients in flow properties normal to the inviscid streamline direction have a negligible effect on the streamwise flow.

$$
\text { Slight rearrangement of the } \eta \text {-momentum equation (equation 4) }
$$
using equation 13 gives 


$$
\begin{aligned}
\frac{u}{e_{1}} \frac{\partial v}{\partial \zeta}+\frac{v}{e_{2}} \frac{\partial v}{\partial \eta}+\left[w+\frac{\left(\overline{\rho^{\prime} w^{\prime}}\right)}{\rho}\right] \frac{\partial v}{\partial \zeta}-\frac{1}{\rho} \frac{\partial}{\partial \zeta}\left[\mu \frac{\partial v}{\partial \zeta}-\rho\left(\overline{v^{\prime} w^{\prime}}\right)\right] \\
-k_{1} u v=-k_{2} u_{e}^{2}\left[\frac{u^{2}}{u_{e}^{2}}-\frac{\rho_{e}}{\rho}\right]
\end{aligned}
$$

Considering the boundary conditions $v_{e}=v_{w}=\left(\overline{v^{\top} w^{\top}}\right)_{e}=\left(\overline{v^{\top} w^{\top}}\right)_{w}=0$, Vaglio-Laurin (21) has shown that if the right hand side is zero then equation 14 has the solution $v=\left(\overline{v^{\prime} w^{\prime}}\right)=0$ everywhere. Thus the crossflow is zero if $K_{2}=0$ (true if the streamlines follow geodesics of the surface) or if $\left[\left(u / u_{e}\right)^{2}-\rho_{e} / \rho\right]=0$ which is nearly true for the highly cooled nose portion of a blunt body in hypersonic flow. One may further suspect that if the right hand side of equation 14 is very small then the velocity $v$ may be very small throughout the boundary layer. Thus in some practical applications $v$ and $\left(\overline{v^{\prime} w^{\top}}\right)$ may be expected to be small. Conversely, it is apparent that the assumption of small cross-flow implies that either $k_{2}$ or $\left[\left(u / u_{e}\right)^{2}-\rho_{e} / \rho\right]$ is small.

The assumption of small cross-flow is now introduced. In this assumption $v$ and the lateral derivatives $\partial / \partial \eta \underline{n}$ are considered small compared to $u$. In equation 2 (continuity) the term $\frac{\partial}{\partial \eta}\left(\rho_{e}, v\right)$ is much smaller than the remaining terms and is neglected. All terms containing $v$ in equation $3(\xi$-momentum) and equation 6 (energy) are of the order $v^{2}$ or $v \frac{\partial}{\partial \eta}$ and are neglected. The term $\frac{v}{e_{2}} \frac{\partial v}{\partial \eta}$ is dropped from equation 4 ( $\eta$-momentum) in comparison with the remaining terms which have the same order as $v$.

If one considers the flow along a particular inviscid streamine, the corresponding distance along the surface is given by 


$$
s=\int e_{1} d \xi
$$

in accordance with equations 1 . Equations 2,3,4 and 6 then become Continuity:

$$
\frac{\partial}{\partial s}(\rho u r)+\frac{\partial}{\partial \zeta}\left[\rho r\left(w+\frac{\left(\overline{e^{\top} w^{\top}}\right)}{\rho}\right)\right]=0
$$

s-Momentum:

$$
\begin{gathered}
\rho\left[u \frac{\partial u}{\partial s}+\left[w+\frac{\left(\overline{p^{\prime} w^{\prime}}\right)}{\rho}\right] \frac{\partial u}{\partial \zeta}\right]=\rho_{e} u_{e} \frac{\partial u_{e}}{\partial s} \\
+\frac{\partial}{\partial \zeta}\left[\mu \frac{\partial u}{\partial \zeta}-\rho\left(\overline{u^{\top} w^{\prime}}\right)\right]
\end{gathered}
$$

Energy:

$$
\begin{gathered}
\rho\left[u \frac{\partial H}{\partial s}+\left[w+\frac{\left(\overline{\rho^{\top} w^{\top}}\right)}{\rho}\right] \frac{\partial H}{\partial \zeta}\right]=\frac{\partial}{\partial \zeta}\left[\mu\left(\frac{\partial H}{\partial \zeta}+\frac{1-P_{I}}{P_{r}} \frac{\partial h}{\partial \zeta}\right)\right. \\
\left.-\rho\left(\overline{w^{\top} H^{\top}}\right)\right]
\end{gathered}
$$

$\eta$-Momentum:

$$
\begin{gathered}
\rho[u \\
\left.\frac{\partial v}{\partial s}+\left[w+\frac{\left(\overrightarrow{\rho^{\prime} w^{\prime}}\right)}{\rho}\right] \frac{\partial v}{\partial \zeta}-k_{1} u v+k_{2} u^{2}\right] \\
=\rho e_{e}^{2} k_{2}+\frac{\partial}{\partial \zeta}\left[\mu \frac{\partial v}{\partial \zeta}-\rho\left(\overline{v^{\prime} w^{\prime}}\right)\right]
\end{gathered}
$$

In the above use has been made of equations 12 and 13 , and $e_{2}$ has been redefined as $I$ in order to introduce a notation familiar in treatment of axisymetric boundary layers.

It is apparent that equations 15,16 , and 17 are identical in form 
to the equations for boundary layer flow over an axially symetric body of radius $r$. This is referred to as the "axially symmetric analogy" in the literature (e.g. see reference 3 ). Of course $r$ here has a different meaning and solutions must proceed along a given streamline.

\section{Integral Equations}

Since exact solution of even the simplified partial,differential equations is not possible, approximate treatment of the problem is enhanced by reducing the system to the integral equations of boundary layer flow. This is accomplished in the usual manner by defining the displacement, momentum, and enthalpy convection thicknesses, then integrating the equations from $\zeta=0$ to $\zeta=8$. The resulting equations describe the mean or averaged properties of the turbulent boundary layer, thus eliminating explicit dependence on the unknown laws of turbulent exchange.

The derivation of the integral equations is discussed in Appendix A. Both the small cross-flow case and the general equations are presented there for the sake of completeness. The resulting small cross-flow equations;considering flow along a particular streamline, are s - Momentum:

$$
\frac{d \theta_{11}}{d s}+\left[\left(2+\frac{s^{*}}{\theta_{11}}\right) \frac{1}{u_{e}} \frac{d u_{e}}{d s}+\frac{1}{\rho_{e}} \frac{d \rho_{e}}{d s}+\frac{1}{r} \frac{d r}{d s}\right] \theta_{11}=\frac{\tau_{1}}{\rho_{e} u_{e}^{2}}
$$

Energy:

$$
\frac{d \varphi_{1}}{d s}+\left[\frac{1}{H_{e}^{*}} \frac{d H_{e}^{*}}{d s}+\frac{1}{u_{e}} \frac{d u_{e}}{d s}+\frac{1}{\rho_{e}} \frac{d \rho_{e}}{d s}+\frac{1}{r} \frac{d r}{d s}\right] \varphi_{1}=\frac{-q_{w}}{\rho_{e} u_{e} H_{e}^{*}}
$$


$\eta$-Momentum:

$$
\begin{gathered}
\frac{d \theta_{2 l}}{d s}+\left[\frac{2}{u_{e}} \frac{d u_{e}}{d s}+\frac{1}{\rho_{e}} \frac{d \rho_{e}}{d s}+\frac{2}{r} \frac{d r}{d s}\right] \theta_{2 l}+k_{2}\left(\theta_{11}+s_{1}^{*}\right) \\
=\frac{\tau_{2} w}{\rho_{e} u_{e}^{2}}=\frac{\tau_{w}}{\rho_{e} u_{e}^{2}} \tan a
\end{gathered}
$$

where

$$
\begin{aligned}
& \theta_{11}=\int_{0}^{\delta}\left(1-\frac{u}{u_{e}}\right) \frac{\rho u}{\rho_{e} u_{e}} d \zeta \quad \delta_{1}^{*}=\int_{0}^{\delta}\left(1-\frac{\rho u}{\rho_{e} u_{e}}\right) d \zeta \\
& \theta_{21}=-\int_{0}^{\delta} \frac{\rho u v}{\rho_{e} u_{e}^{2}} d \zeta \quad \Phi_{1}=\int_{0}^{\delta} \frac{\rho u}{\rho_{e} u_{e}}\left(1-\frac{H^{*}}{H_{e}^{*}}\right) d \zeta
\end{aligned}
$$

$$
\mathrm{H}^{*}=\mathrm{H}-\mathrm{H}_{\mathbf{w}}
$$

and $\tau_{1_{w}}$ and $\tau_{2_{w}}$ are the wall shear stresses in the $s$ and $\eta$ directions, respectively. Use has been made of the definition of the angle a between the inviscid streamline and the limiting streamline (direction of the velocity vector as the surface is approached) in equation 21. That is

$$
\tan a \equiv \lim _{\zeta \rightarrow 0} \frac{v}{u}=\frac{\tau_{2}}{\tau_{1}}
$$

Again it is noted that equations 19 and 20 are precisely analogous to the momentum integral and energy integral equations for flow over an axisymmetric body and are independent of the $\eta$-momentum equation. 


\section{Solution Considerations}

The introduction of the small cross-flow assumption results in significant simplification of the three-dimensional problem as has been demonstrated in the preceding development. The existence of the axially symmetric analogy permits one to take advantage of experience gained from treatment of two-dimensional problems in obtaining solutions to certain classes of three-dimensional flows, namely those for which the small cross-flow assumption is reasonable. This point is especially important for turbulent boundary layers, since useful solutions are of necessity based on key assumptions resulting from experimental evidence and experience.

The validity of the small cross flow assumption is in general not known a priori for many practical problems. (In this sense a solution to the linearized $\eta$-momentum equation is of value, if for no other purpose than to indicate when this postulate may be in error for a particular example.) Justification for basing a computation method on this assumption must in the final analysis rest in comparisons with experiment. However, several considerations lend support to its utility in the turbulent case. Of course the cross-flow is known to be small in many applications such as, for example, the forward portion of highly cooled blunt bodies considered in reference 21 .

It has been noted that the cross-flow in a turbulent boundary layer is much less than in a laminar layer. This is a result of the larger apparent shear stress which tends to reduce the growth of crossflow as has been discussed in some detail by Cooke (4). In this sense, computations reported by Beckwith (29) for laminar flow over a yawed 
infinite cylinder lend some further encouragement as to the usefulness of small cross-flow solutions. Here small cross-flow results were compared with exact laminar solutions for a yawed cylinder. It was concluded that the small cross-flow solutions provided reasonable approximations to the velocity profiles and skin friction even when the crossflow velocity was much larger than specified by the theoretical criterion limiting the validity of the assumption.

While the above comments give some support to the further deve1opment of theory based on small secondary flow, it is noted that in many instances such an assumption could not be considered reasonable. For example, flow in the vicinity of a separation line would certainly not be expected to have negligible cross-flow effects.

Consider now the solution of the simplified integral equations, equations 19,20 , and 21 , along a particular streamline. First the geometric properties $\frac{1}{r} \frac{d r}{d s}$ and $K_{2}$ must be determined from the inviscid flow and the surface geometry under consideration. Then the streamwise solution (equations 19 and 20 ) may be obtained employing approximate methods of known validity for axially symmetric flow. Finally the $\eta$-momenturn equation may be treated after an appropriate cross-flow velocity profj.le is assumed.

The application of two-dimensional (or axisymmetric) methods to the streamwise equations assumes implicitly that this component of the flow develops as would an axially symmetric one under the influence of the streamwise pressure gradient. Recent measurements by Hall (24) in compressible, three-dimensional flow give qualitative support to this postulate. Hall observed that the shapes of his measured streamwise 
velocity profiles react to pressure gradients (both favorable and adverse) in the same sense found in two-dimensional boundary layers. Thus, as indicated by Hall, one would expect that a computation method which takes into account the effect of pressure gradient on the streamwise velocity profile may be of value. This is an important consideration in the method of solution formulated in the next chapter.

The solution of the $\eta$-momentum equation is dependent on the choice of a form for the cross-flow velocity profile. This point is discussed in detail in the following chapter where a solution technique of general applicability is developed. 


\title{
CHAPTER III
}

\section{METHOD OF SOLUTION}

\begin{abstract}
The approximate solution of the small cross-flow integral equations is treated in this chapter. A general technique is proposed which is applicable to flows with streamwise pressure gradients over adiabatic surfaces or isothermal surfaces with heat transfer. Attention is drawn to the restrictive assumptions employed and the resulting limitations. First, solution of the streamwise flow is considered. Then the cross-flow momentum equation is treated consistent with and subject to the streamwise flow solution. Its solution is discussed along with the important velocity profile approximation involved. Finally, a summary of the proposed calculation method is given outlining the key postulates.
\end{abstract}

\section{Streamwise Flow}

Taking full advantage of the mathematical analogy between the streamwise flow and flow about an axisymmetric body, a computation method of proven utility for two-dimensibnal flows is applied directly to the problem at hand. While calculation procedures for the compressible turbulent boundary layer have been suggested by many workers, as for example, Reshokto and Tucker (30), Spence (23), Cohen (31), and Standen (32), a method given recently by Sasman and Cresci (33) has been selected for the present application. The wide range of applicability of this technique has been demonstrated in reference 33 for favorable and adverse pressure gradients, with and without heat transfer. 
Sasman and Cresci express the boundary layer in terms of the : momentum integral and moment-of-momentum integral equations for arbitrary pressure gradient. A transformation simplifies the equation set, and the coupled equations are solved simultaneously to yield the thickness functions, skin friction, and related quantities. The main features of this method are outlined below in the present context, i.e. considering the flow along a given inviscid streamline path for which the physical distance: along the surface is given by $s$.

A transformation of the Mager (25) type is applied in the form

$$
s=\int_{0}^{s} a(s) d s
$$

and

$$
z=b(s) \int_{0}^{\zeta} \frac{\rho}{\rho_{0}} d \zeta
$$

where $S$ and $Z$ are the transformed coordinates. Also

$$
a=\frac{T_{0}}{T_{I}}\left(\frac{T_{e}}{T_{0}}\right)^{\frac{\varphi+1}{2(\gamma-1)}}
$$

and

$$
b=\left(\frac{T_{e}}{T_{0}}\right)^{\frac{1}{2}}
$$

The Ludwieg-Tillmann (34) skin friction law for incompressible flow is adapted for compressible flow using the reference temperature concept 
which yields

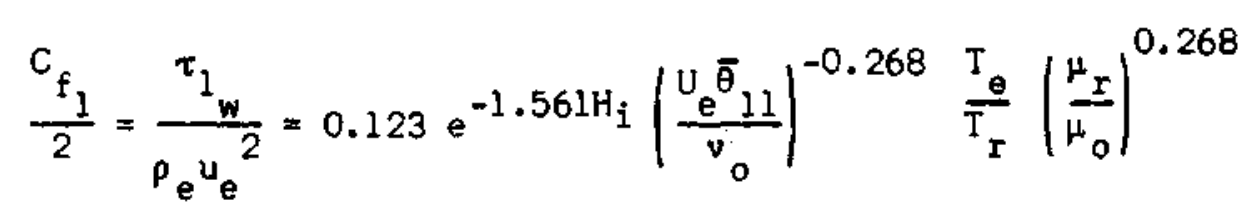

where $C_{f_{l}}$ is the streamwise component of skin friction coefficient, $U$ and $\bar{\theta}_{11}$ are the transformed streamwise velocity and momentum thickness, and where the subscript $r$ refers to conditions evaluated at an appropriate reference temperature, as for example that given by Eckert (35). As Sasman and Cresci note, the derivation of this friction law is not restricted to zero pressure gradient flows (see reference 36).

Results of equilibrium turbulent boundary layer analyses given by Libby et al. (37) were employed to evaluate a normalized shear integral appearing in the moment-of-momentum equation. Further, a Crocco velocitytemperature relation was assumed along with a power velocity law with Prandtl number equal unity. Thus

$$
\frac{H^{*}}{H_{e}^{*}}=\frac{u}{u_{e}}=\frac{U}{U_{e}}
$$

and

$$
\frac{\mathrm{u}}{\mathrm{U}_{\mathrm{e}}}=\left(\frac{\mathrm{Z}}{\Delta}\right)^{\mathrm{N}_{1}}
$$

where $\Delta$ is the boundary layer thickness in the transformed plane.

The following thickness functions are defined in the transformed plane: 


$$
\begin{gathered}
\bar{\theta}_{11}=\int_{0}^{\Delta} \frac{U}{U_{e}}\left(1-\frac{U}{U_{e}}\right) d z \\
\Delta_{1}^{*}=\int_{0}^{\Delta}\left(1-\frac{U}{U_{e}}\right) d z+\int_{0}^{\Delta}\left(\frac{H}{\hat{H}_{e}}-1\right) d z \\
H_{1}=\frac{1}{\tilde{\theta}_{11}} \int_{0}^{\Delta}\left(1-\frac{U}{U_{e}}\right) d z
\end{gathered}
$$

where $H_{i}$ is the transformed form factor for adiabatic flow. One notes fromt equations 30 and 32 that the exponent in the assumed power law velocity profile (equation 29 ) is related to $H_{i}$ by

$$
\mathrm{N}_{i}=\frac{\mathrm{H}_{i}-1}{2}
$$

Resulting, relations between the transformed and compressible boundary layer parameters are

$$
\begin{gathered}
u=\left(\frac{T_{e}}{T_{0}}\right)^{\frac{1}{2}} U \\
H_{c}=\frac{T_{w}}{T_{e}} H_{1}+\frac{T_{0}}{T_{e}}-1 \\
\theta_{11}=\left(\frac{T_{o}}{T_{e}}\right)^{\frac{r+1}{2(r-1)}} \tilde{\theta}_{11}
\end{gathered}
$$

and 


$$
\delta_{1}^{*}=\left(\frac{\mathrm{T}_{0}}{\mathrm{~T}_{e}}\right)^{\frac{3 \gamma-1}{2(\gamma-1)}}\left(\vec{\theta}_{11}+\Delta_{1}^{*}\right)-\left(\frac{\mathrm{T}_{0}}{\mathrm{~T}_{e}}\right)^{\frac{r-1}{2(r-1)}} \bar{\theta}_{11}
$$

where $H_{c}$ is the compressible form factor $8{ }_{1}^{*} / \theta_{11}$.

A parameter $f$ is defined by

$$
f \equiv\left(\frac{e^{\bar{\theta}} 11}{v_{0}}\right)^{1.268}=\left(\frac{M_{e_{0}} \bar{\theta} 11}{v_{0}}\right)^{1.268}
$$

where $a_{0}$ is the sound velocity, $\sqrt{Y^{R T}}$, and $R$ is the gas constant. The: momentum and moment-of-momentum integral equations of reference 33 are now written in terms of the physical distance coordinate $s$ as

$$
\frac{d f}{d s}=1.268\left[\frac{-f}{M_{e}} \frac{d M_{e}}{d s}\left(1+\frac{T_{w}}{T_{0}} H_{i}\right)-\frac{f}{r} \frac{d r}{d s}+B\right]
$$

and

$$
\begin{aligned}
\frac{d H_{i}}{d s}=-\frac{1}{2 M_{e}} \frac{d M_{e}}{d s}\left[H_{i}\left(H_{i}+1\right)^{2}\left(H_{i}-1\right)\right]\left[1+\left(\frac{T_{w}}{T_{0}}-1\right) \frac{H_{i}^{2}+4 H_{i}-L}{\left(H_{i}+1\right)\left(H_{i}+3\right)}\right] \\
+\frac{\left(H_{i}^{2}-1\right)}{f} B\left[H_{i}-\frac{0.011\left(H_{i}+1\right)\left(H_{i}-1\right)^{2}}{H_{i}^{2}} \frac{2}{C_{f_{1}}} \frac{I_{0}}{T_{I}}\right]
\end{aligned}
$$

where

$$
B=0.123 e^{-1.561 H_{i}} \frac{M_{e} a_{0}}{v_{0}} \frac{T_{e}}{T_{r}}\left(\frac{T_{e}}{T_{0}}\right)^{3}\left(\frac{\mu_{r}}{\mu_{0}}\right)^{0.268}
$$

and $Y=1.4$. 
For the details of the derivation the reader is referred to reference 33. The above equations are a direct consequence of applying the development of this reference to equations 15, 16, and the moment-of momentum equation corresponding to equation 16.

Now upon specification of the external flow field along the inviscid streamline and the streamline divergence term $\frac{1}{r} \frac{d r}{d s}$ from the geometric properties of the coordinate system, simultaneous solution of equations 39 and 40 yield $\mathrm{H}_{j}(\mathrm{~s})$ and $\bar{\theta}_{11}(\mathrm{~s})$. From these results the desired properties of the compressible flow are readily obtained using equations 27, 35, and 36. The heat transfer may be computed from $C_{f_{1}}$ if a form of Reynolds analogy is assumed. This assumption is discussed more fully in a later section.

The principal advantage of the method of Sasman and Cresci when compared with other approximate calculation procedures stems from a more complete account of the effect of pressure gradient on the solution. Both the skin friction law and the velocity profile relation are explicitly related to $H_{i}$ which in turn becomes a dependent variable in the coupled differential equations. An earlier method developed by Reshotko and Tucker $(30)$ treated the problem in a similar manner but apparently restricted the range of applicability by uncoupling the momentum and moment-of-momentum equations.

As is the case in calculation methods for the turbulent boundary layer, a number of approximations based mainly on experimental evidence are required in order to effect a solution. These have been noted for the present method in the preceding paragraphs. Perhaps the most questionable of these is the assumed Crocco relation (equation 28) for flows with pressure gradient and heat transfer. This relation, strictly valid for adiabatic flows with Prandtl number of unity, is used to 
evaluate certain enthalpy integrals in lieu of solving the energy equation. (See reference 33 for details.) Rotta (38), for example, has reported that no evidence supporting the validity of the Crocco relation was to be found in velocity and temperature profile data which he examined for supersonic turbulent boundary layers with heat transfer. However, good quantitative agreement of the computed thickness functions and skin friction coefficient with experiment was reported by Sasman and Cresci (33) for a wide variety of problems.

\section{Cross-Flow}

\section{Iransformation}

Once the streamwise solution has been obtained the $\eta$-momentum equation may be solved dependent upon the specification of the cross-flow velocity profile. The equation is first simplified employing the transformation of Sasman and Cresci as given in the previous section. Cooke and Hall (3) have noted that although a compressibility correlation of the Stewartson type does not hold for general three-dimensional flows, it does hold for: small cross-flow. A demonstration of this correlation was given for the laminar momentum equation in reference 3 . In Appendix 3 the turbulent $\eta$-momentum equation (equation 18) is shown to reduce to an incompressible form upon application of the transformation of Sasman and Cresci and the corresponding transformed momentum integral equation is given.

The resulting transformed momentum integral equation is

$$
\frac{d \bar{\theta}_{21}}{d S}+\bar{\theta}_{21}\left(\frac{2}{\bar{U}_{e}} \frac{d U_{e}}{d S}+\frac{2}{r} \frac{d r}{d S}\right)+\bar{K}_{2}\left(\bar{\theta}_{11}+\Delta_{1}^{*}\right)=\frac{1}{a} \frac{\tau_{2}}{\rho_{o} U_{e}^{2}}
$$


where

$$
\bar{\theta}_{21}=-\int_{0}^{\Delta} \frac{u v}{u_{e}^{2}} d z
$$

and $\Delta$ is the boundary layer thickness in the transformed plane. The following relations are observed in the transformation

$$
\begin{gathered}
v=v \\
K_{2}=\left(\frac{T_{e}}{T_{o}}\right)^{\frac{r}{\gamma-1}} \frac{T_{0}}{T_{T}} \bar{K}_{2}
\end{gathered}
$$

and

$$
\theta_{12}=\left(\frac{T_{0}}{\bar{T}_{\mathrm{e}}}\right)^{\frac{\gamma}{Y-1}} \bar{\theta}_{12}
$$

Now considering the definition of the angle between the inviscid streamline and the limiting streamline, equation 22, one notes that in the transformed plane

$$
\tan \bar{a}=\lim _{z \rightarrow 0} \frac{V}{U}=\left(\frac{T_{e}}{T_{0}}\right)^{\frac{1}{2}} \tan \alpha
$$

where $\bar{a}$ is the transformed equivalent of a.

Thus

$$
\tau_{2_{w}}=\tau_{1_{w}} \tan \alpha=\left(\frac{\mathrm{T}_{0}}{\mathrm{~T}_{\mathrm{e}}}\right)^{\frac{1}{2}} \tau_{1_{w}} \tan \bar{\alpha}
$$


Use of the Crocco relation to evaluate the enthalpy integral in $\Delta_{1}^{*}$, equation 31, then permits the transformed momentum-integral equation to be written as

$$
\begin{gathered}
\frac{d \bar{\theta}_{21}}{d S}+\bar{\theta}_{21}\left(\frac{2}{U_{e}} \frac{d U_{e}}{d S}+\frac{2}{r} \frac{d r}{d S}\right)+\bar{K}_{2} \bar{\theta}_{11}\left(1+\frac{T_{w}}{T_{0}} H_{1}\right) \\
=\frac{T_{r}}{T_{0}} \frac{C_{f_{1}}}{2} \tan \bar{a}
\end{gathered}
$$

where equations 25 and 27 have been employed. The cross-flow momentum equation has now been set in the transformed plane consistent with the streamwise solution. Equation 49 is in exact incompressible form for adiabatic flow if the incompressible $C_{f}$ is equal to $\left(T_{r} / T_{0}\right) C_{f_{1}}$. This is the same relation obtained by Sasman and Cresci. (For example, compare equation 49 with equation 16 of reference 33.)

\section{Profile Approximation}

Solution of equation 49 is dependent upon the specification of the cross-flow velocity profile. The precise form that this profile should take is uncertain, due primarily to the very small body of experimental data available. Cooke (4) has reviewed the various assumed profile relations used by several workers, mainly in incompressible flow. In the present application a velocity profile is assumed in the transformed plane of the form

$$
\frac{v}{U_{e}}=\left(1-\frac{Z}{\Delta}\right)^{2} \frac{U}{U_{e}} \tan \bar{a}
$$

and is referred to as the "parabolic" relation. This form has been used 
by Mager (10) and Cooke (11) in incompressible flows and by Braun (20) for the compressible case of a cone at small yaw. Experimental data of Gruschwitz (14) for a curved channel and Wallace (17) for a swept, tapered wing serve as basis for the parabolic relation. Cooke (4) notes that this form also fits the experiments of Kuethe et al. (15) very well.

Hall (24) has recently reported measurements of the cross-flow velocity profile for flow in a supersonic nozzle where the pressure along an inviscid streamline first decreased then increased. These data indicate that the parabolic relation provides a reasonable approximation in the favorable pressure gradient region but is in error in the region of adverse pressure gradient. In view of these results the parabolic relation will be generally considered to be limited in applicability to favorable streamwise pressure gradients. Of course more careful experimentation is required to estabiish definite limits for this approximation.

In the following chapter, where the method is applied to a specific example, the profile approximation is treated as a parameter in order to assess its effect on the solution of the momentum integral equation. In particular, "cubic" and "linear" approximations are assumed and solutions are compared with ones resulting from the parabolic relation.

Now the profile approximation given by equation 50 is employed, and equation 21 is put into a form for solution consistent with the streamwise equations. The details are given in Appendix $C$. The resulting equation in the physical distance coordinate $s$ is 


$$
\begin{aligned}
\frac{d A}{d s}=-A & {\left[\frac{1}{1.268 f} \frac{d f}{d s}+\frac{1}{M_{e}} \frac{d M_{e}}{d s}+\frac{2}{r} \frac{d r}{d s}-\frac{\left(2 H_{i}+1\right)}{\left(H_{i}-1\right)\left(H_{i}+2\right)} \frac{d H_{i}}{d s}\right.}
\end{aligned}
$$

where $A$ is defined by

$$
A=\tan \bar{a}
$$

and where $f$ and $B$ are given by equations 38 and 41 , respectively. Solution of equation 51 is possible when the streamwise solution (equations 39 and 40 ) is given along with the geometric relation $K_{2}(s)$. The solution yields $A(s)$ which is related to the angle a between the inviscid streamline and the limiting streamline by equations 47 and 52 . Significance of the Solution

At this point some comments on the significance of the solution to equation 51 are in order. The cross-flow solution is of value in that it serves as an indication of the validity of the small cross-flow assumption. In order for the cross-flow to be considered small the physical quantity given by the ratio of the local cross-flow velocity to the streamwise velocity, $v / u$, must be small. In particular the inequality

$$
\left(\frac{\mathrm{v}}{\mathrm{u}}\right)^{2} \ll 1
$$

must be satisfied. Considering the parabolic profile assumption, or more generally any cross-flow profile of the form

$$
\frac{v}{U_{e}}=\left(1+\frac{Z}{\Delta}\right)^{n} \frac{U}{U_{e}} \tan \bar{a}
$$


where $n>0$, it is immediately apparent that $(v / u)_{\text {max }}$ occurs as $z / \Delta \rightarrow 0$, i.e. as the surface is approached, and is given by

$$
\left(\frac{v}{u}\right)_{\max }=\lim _{z / \Delta \rightarrow 0}\left(1-\frac{z}{\Delta}\right)^{n} \tan \alpha=\tan \alpha
$$

The solution $A(s)$ then gives a direct measure of the physical restraint necessary for the small secondary flow assumption to be applicable as given by equation 53 . This restraint may be rewritten in terms of the maximum value of $v / u$ as

$$
\frac{T_{0}}{T_{e}} A^{2}<<1
$$

In many practical applications predetermination of the magnitude of the cross-flow is not possible. In such instances'solutions of equation 51 in conjunction with the small cross-flow streamwise equations (equations 39 and 40 ) indicate where the assumption may be in substantial error.

From the definition of a one notes that when the component o: skin friction coefficient in the $\eta$-direction is defined by

$$
\frac{\mathrm{C}_{f_{2}}}{2}=\frac{\tau_{2}}{\rho_{\mathrm{w}} \mathrm{u}_{\mathrm{e}}^{2}}
$$

then

$$
\frac{C_{f_{2}}}{C_{f_{1}}}=\tan a=\left(\frac{T_{0}}{T_{e}}\right)^{\frac{1}{2}} A
$$


The crosswise component of skin friction coefficient is thus determined by the cross-flow solution.

One may be tempted at this point to compute a second approximation to the three-dimensional skin friction coefficient by simple vector addition of the force components corresponding to $\mathrm{C}_{\mathrm{f}_{1}}$ and $\mathrm{C}_{\mathrm{f}_{2}}$. This step is of questionable value because of the small cross-flow approximation. For example, define a resultant skin friction coefficient $C_{f}^{*}$ whose magnitude is given by

$$
c_{f}^{*}=c_{f_{1}} \sqrt{1+\left(\frac{C_{f_{2}}}{C_{f_{1}}}\right)^{2}}=c_{f_{1}}\left[1+\frac{\tan ^{2} a}{2}+\left(\begin{array}{l}
\text { higher } \\
\text { order } \\
\text { terms }
\end{array}\right)\right]
$$

Now, since $\tan \alpha$ is equal to $(v / u)_{\text {max }}$ (equation 55 ), it is seen that the correction term $\frac{\tan ^{2} \alpha}{2}$ is of the same order of magnitude as the terms eliminated from the $\xi$-momentum equation by the small cross-flow assumption: This means that the correction afforded by $\frac{\tan ^{2} \alpha}{2}$ may be of the same order of magnitude as the error introduced into the streamwise component $C_{f_{1}}$ by the small cross-flow approximation. Therefore, $C_{f}^{*}$ is no better as an approximation for the resultant skin friction than the basic small cross-flow approximation which implies that $C_{f}^{*}=C_{f_{1}}$.

\section{Summary}

The steps to be followed in solving the governing equations developed in the preceding sections are outlined here. It is convenient to write the equations in non-dimensional form for numerical solution. Thus a reference length $L$ is taken as some characteristic length of 
the particular flow under consideration. Let $\bar{s}=s / L$ be the nondimensional distance along the surface, define $K$ as the non-dimensional curvature parameter given by

$$
K=K_{2} L\left(\frac{T_{0}}{T_{e}}\right)^{\frac{1}{2}}
$$

and define a reference Reynolds number by

$$
R_{0}=\frac{L_{0}}{v_{0}}
$$

The governing equations for the streamwise flow are written in the form

$$
\frac{d f}{d \bar{s}}=-1.268\left[\frac{f}{M_{e}} \frac{d M_{e}}{d \bar{s}}\left(1+\frac{T_{w}}{T_{0}} H_{i}\right)+\frac{f}{r} \frac{d r}{d \bar{s}}-C\right]
$$

and

$$
\begin{aligned}
& \frac{\mathrm{dH}_{i}}{\mathrm{~d} \overline{\mathrm{s}}}=\frac{-1}{2 \mathrm{M}_{e}} \frac{\mathrm{d} \mathrm{M}_{e}}{\mathrm{~d} \overline{\mathrm{s}}}\left[\mathrm{H}_{i}\left(\mathrm{H}_{i}+1\right)^{2}\left(\mathrm{H}_{i}-1\right)\right]\left[1+\left(\frac{\mathrm{T}_{\mathrm{w}}}{\mathrm{T}_{0}}-1\right) \frac{\left(\mathrm{H}_{i}^{2}+4 \mathrm{H}_{i}-1\right)}{\left(\overline{\left.H_{i}+1\right)\left(\bar{H}_{i}+3\right)}\right]}\right. \\
& +\frac{\left(H_{i}^{2}-1\right)}{f} H_{i} C-0.011 \frac{\left(H_{i}-1\right)^{2}\left(H_{i}^{2}-1\right)\left(H_{i}+1\right)}{H_{i}^{2} f^{0.789}}\left(\frac{T_{e}}{T_{0}}\right)^{3} \frac{T}{0}_{T} R_{0} M_{e}
\end{aligned}
$$

where

$$
C=0.123 e^{-1.561 H_{i}} R_{0} M_{e} \frac{T_{e}}{T_{r}}\left(\frac{T_{e}}{T_{0}}\right)^{3}\left(\frac{\mu_{r}}{H_{0}}\right)^{0.268}
$$

and 


$$
f \equiv\left(\frac{\mathrm{e}^{\bar{\theta}} 11}{v_{0}}\right)^{1.268}=\left(M_{e} R_{0} \frac{\bar{\theta}_{11}}{L}\right)^{1.268}
$$

The cross-flow equation becomes

$$
\begin{aligned}
\frac{d A}{d \bar{s}}= & -A\left[\frac{1}{1.268 f} \frac{d f}{d \bar{s}}+\frac{1}{M_{e}} \frac{d M_{e}}{d \bar{s}}+\frac{2}{r} \frac{d r}{d \bar{s}}-\frac{\left(2 H_{i}+1\right)}{\left(H_{i}-1\right)\left(H_{i}+2\right)} \frac{d H_{i}}{d \bar{s}}\right. \\
& \left.+\frac{\left(H_{i}-1\right)\left(H_{i}+2\right)}{2 f} c\right]+K \frac{\left(H_{1}-1\right)\left(H_{i}+2\right)}{2}\left(1+\frac{I_{w}}{\bar{I}_{0}} H_{i}\right)
\end{aligned}
$$

Recalling that these equations are valid along a particular inviscid streamline, solution of the problem requires the specification of the streamline divergence term $\frac{1}{r} \frac{d r}{d s}$ and the curvature parameter $K$ along with $M_{e}$ and $I_{e} / T_{0}$ as a function of $\bar{s}$. The parameters $\frac{1}{r} \frac{d r}{d \bar{s}}$ and $K$ as well as the path traced by the inviscid streamline on the surface are intrinsically related to the differential geometry of the surface and the inviscid flow so that convenient methods for determining their values may vary widely for specific applications. A discussion of this point is not intended here. However, it is noted that Vaglio-Laurin (39) has outlined a method for numerically computing these relations which is quite general in application.

Specification of initial values for $f\left(\right.$ or $\left.\theta_{11}\right), H_{i}$, and $A$ now permits integration of equations 62,63 and 64 . Initial conditions will naturally depend on the flow being considered and may be estimated from experiment in some applications or may be related to laminar conditions at some known or estimated transition location. Simultaneous integration of equations 62 and 63 then yields the distribution of $\theta_{11}$ and $H_{i}$ from 
which the related boundary layer parameters may be computed. Integration of equation 64 , subject to the streamwise solution, gives $A(s)$.

In applying an approximate calculation procedure such as the one suggested above it is important that the approximations involved be kept firmly in mind. In view of this fact the key assumptions used in the present development are listed below.

(1) perfect gas, $r=1.4$

(2) small secondary flow

(3) power streamwise velocity profile, equation 29

(4) Crocco relation between streamwise velocity and temperature, equation 28

(5) Ludwieg-Tillmann friction law adapted to compressible flow by the reference temperature, equation 27

(6) parabolic cross-flow velocity relation, equation 50

In the next chapter the method is illustrated by application to a specific example, the yawed infinite cylinder. Some of the parameters which affect the cross-flow are investigated. 


\section{CHAPTER IV}

\section{RESULTS FOR A YAWED INFINITE CYL INDER}

The computation method described in the last chapter is applied to the particular case of a yawed infinite cylinder of circular crosssection. This example is chosen to illustrate an application of the small cross-flow theory because the basic features of three-dimensional flow are present, including cross-flow which would not necessarily be expected to be small, while the geometric properties are not complex. In addition, experimental heat transfer data have been reported by Beckwith and Gallagher (22) for this case, thus allowing comparisons of the theory with experiment.

The flow conditions for the numerical calculations which follow have been chosen to correspond with those of reference 22 . In this reference Beckwith and Gallagher measured the local heat transfer, equilibrium temperatures, and wall static pressure on a circular cylinder at yaw angles of $0,10,20,40$, and 60 degrees. The free stream Mach number was 4.15 and the Reynolds numbers based on cylinder diameter ranged from $1 \times 10^{6}$ to $4 \times 10^{6}$. Heat transfer measurements were recorded using heat meters installed in the surface of the internally cooled model. The cylinder model spanned the 12 inch test section of a supersonic wind tunnel, and end plates were installed to simulate a cylinder of infinite extent.

In the following sections the required inviscid flow and geometric 
properties are calculated. The streamwise solutions are then presented for flow along the stagnation streamline and over the forward portion of the swept cylinder. These results are compared with experimental heat transfer data. Finally, solutions of the cross-flow momentum equation are presented, and the effects of several parameters on these solutions are demonstrated.

\section{Inviscid Flow and Geometric Relations}

Consider a cylinder of infinite extent placed in a supersonic stream at an angle of yaw $\Lambda$ defined by the angle between the free stream velocity vector and the in-plane normal to the cylinder axis. An orthogonal coordinate system is defined on the surface of the cylinder by $\bar{y}$ measured along the generators of the cylinder and $\bar{x}$ measured chord-wise from the stagnation line (perpendicular to the generators) as shown in Figure 2. The velocity components in the $\bar{x}, \bar{y}$ system are given by $\bar{u}, \bar{v}$, respectively. For a specified free stream Mach number $M_{b o}$ and yaw angle $\Lambda$ the inviscid flow conditions on the stagnation line are readily obtained from the shock wave and/or insentropic flow relations of reference 40. The required properties are given by

$$
\begin{aligned}
& \frac{p_{5 l}}{p_{0}}=\left[\frac{(Y-1) M_{\infty}^{2}+2}{(Y-1) M_{\infty}^{2}+2}\right]^{\frac{\gamma}{Y-1}} \\
& M_{s l}^{2}=\frac{2}{Y-1}\left[\frac{(Y-1) M_{\infty}^{2}+2}{(Y-1) M_{\infty}^{2}+2}-1\right]
\end{aligned}
$$




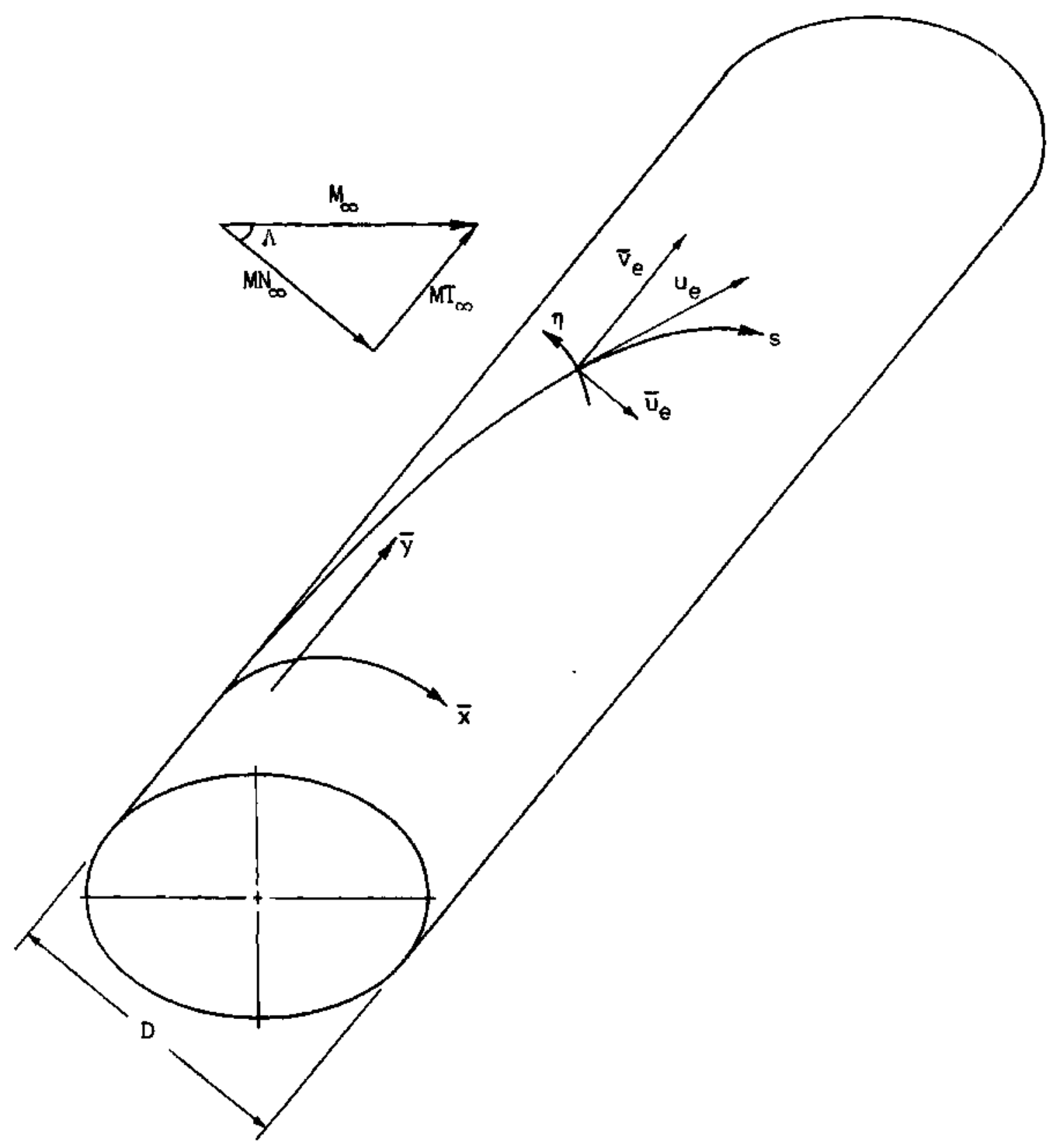

Figure 2. Surface Coordinates on a Yawed Infinite Cylinder. 
and

$$
\frac{T_{s l}}{T_{0}}=\left[1+\frac{r-1}{2} M_{s l}^{2}\right]^{-1}
$$

where the subscript sl refers to the stagnation line, $T_{0}$ is the total temperature of the flow, $\mathrm{MN}_{\infty}$ is the component of free stream Mach number normal to the cylinder leading edge, and $p_{0}$ is the free stream total pressure if $M_{\infty}<1$ or the total pressure behind the shock wave if $\mathrm{MN}_{\infty}>1$. The flow behind the shock wave is assumed isentropic and the gas is assumed perfect. For a given distribution of pressure over the surface of the cylinder $p_{e}(\bar{x})$ the values $M_{e}(\bar{x})$ and $T_{e}(\bar{x})$ are given by the following isentropic flow relations.

$$
M_{e}=\frac{2}{r-1}\left[\left(\frac{p_{0}}{p_{e}}\right)^{\frac{r-1}{r}}-1\right]
$$

and

$$
\frac{T_{e}}{T_{0}}=\left[1+\frac{Y-1}{2} M_{e}^{2}\right]^{-1}
$$

where as before the subscript e refers to local values external to the boundary layer.

Now the streamline coordinate $s(\bar{x}, \bar{y})$ as illustrated in Figure 2 and the geometric properties $\frac{1}{r} \frac{d r}{d s}$ and $K_{2}$ along this coordinate are to be determined. The expressions for these parameters are derived in Appendix $D$ and the resulting relations are given below. As shown in Figure 2 the non-dimensional velocity components are 


$$
\begin{aligned}
& \frac{\bar{v}_{e}}{a_{0}}=M_{\infty}\left(\frac{T_{\infty}}{T_{0}}\right)^{\frac{1}{2}} \sin \Lambda=M_{s l}\left(\frac{T_{s l}}{T_{0}}\right)^{\frac{l}{2}} \\
& \frac{\bar{u}_{e}}{a_{0}}=\left[M_{e}^{2} \frac{T_{e}}{T_{0}}-\left(\frac{\bar{v}_{e}}{a_{0}}\right)^{2}\right]^{\frac{1}{2}}
\end{aligned}
$$

and the resultant non-dimensional velocity is

$$
\frac{u_{e}}{a_{0}}=M_{e}\left(\frac{T_{e}}{T_{0}}\right)^{\frac{1}{2}}
$$

where $a_{0}=\sqrt{r \mathrm{RT}_{0}}$.

On the stagnation line $u_{e}=\bar{v}_{e}=$ constant, $\bar{u}_{e}=0, s=\bar{y}$.

Furthermore as shown in Appendix D

$$
\left(\frac{1}{r} \frac{d r}{d s}\right)_{s l}=\frac{1}{\bar{v}_{e}}\left(\frac{d \bar{u}_{e}}{d \bar{x}}\right)_{s \ell}
$$

and

$$
\left(k_{2}\right)_{s} l^{\prime}=0
$$

Over the remainder of the cylinder $s(\bar{x}, \vec{y})$ is given by the relations

$$
\frac{d s}{d \bar{x}}=\frac{u_{e}}{\bar{u}_{e}} \quad \frac{d \bar{y}}{d \bar{x}}=\frac{\tilde{v}_{e}}{\bar{u}_{e}}
$$

and the divergence and curvature parameters are 


$$
\frac{1}{r} \frac{d r}{d s}=\frac{\bar{v}_{e}^{2}}{u_{e}^{3}} \frac{d \bar{u}_{e}}{d \bar{x}}
$$

and

$$
K_{2}=-\frac{\bar{v}_{e} \bar{u}_{e}}{u_{e}^{3}} \frac{d u_{e}}{d \bar{x}}
$$

It is noted that the relations given here are applicable to a yawed infinite cylinder of any cross-section and not restricted to the circular cylinder of the present example. Upon specification of $M_{\infty}, \Lambda$, and the chord-wise static pressure distribution $p_{e}(\bar{x})$ the inviscid flow and geometric properties required for the boundary layer solution may now be computed.

In the current application $M_{\infty}=4.15$ and yaw angles of $10^{\circ}, 20^{\circ}$, $40^{\circ}$ and $60^{\circ}$ are considered. The experimental pressure distributions of reference 22 were employed to calculate the required parameters numerically on a Burroughs B5500 computer. Zera degree yaw data were used for $\Lambda=10^{\circ}$ since no experimental data were reported for this yaw angle in reference 22. In the immediate vicinity of the stagnation line where a high degree of accuracy for $p_{e}(\bar{x})$ is required for satisfactory determination of $d \bar{u}_{e} / d \bar{x}$ the modified Newtonian relation

$$
\frac{p_{e}}{p_{s l}}=\left(1-\frac{p_{\infty}}{p_{s l}}\right) \cos ^{2}\left(\frac{2 \bar{x}}{D}\right)+\frac{p_{\infty}}{p_{s l}}
$$

was employed. The results of these calculations are presented in Figures 3 through 6. The characteristic length of the flow is taken as the 
cylinder diameter $D$ and the non-dimensional quantities are plotted as a function of the non-dimensional chord-wise surface distance $\bar{x} / \mathrm{L}$ measured from the stagnation line. The flow region of concern is from the stagnation line $\bar{x} / D=0$ to the shoulder of the cylinder $\bar{x} / D=0.785$. The local Mach number distributions are shown in Figure 3 for the four yaw angles. The distance measured along the streamline coordinate $s$ is given as a function of $\bar{x} / D$ in Figure 4 . Here $s / D=\bar{s}$ is measured from a reference point near the stagnation line, in particular $\bar{x} / D=0.01$. The streamline divergence parameter is plotted in Figure 5 where one notes that the maximum divergence occurs on the stagnation line and becomes small as the shoulder is approached. The non-dimensional curvature parameter $K$ as defined by equation 60 is shown in Figure 6 . It is evident that the maximum curvature of the streamline is greater for the lower sweep angles where the peak occurs nearer the stagnation lirie. This is consistent with the high streamline divergence noted in Figure 5 for these low $A$ cases.

\section{Stagnation Line Solutions}

Along the stagnation streamline at the cylinder leading edge the governing integral equations are simplified since the Mach number is constant. Equations 62 and 63 reduce to

$$
\frac{d f}{d \bar{s}}=-1.268\left[f \frac{l}{r} \frac{d r}{d \bar{s}}-c\right]
$$

and 


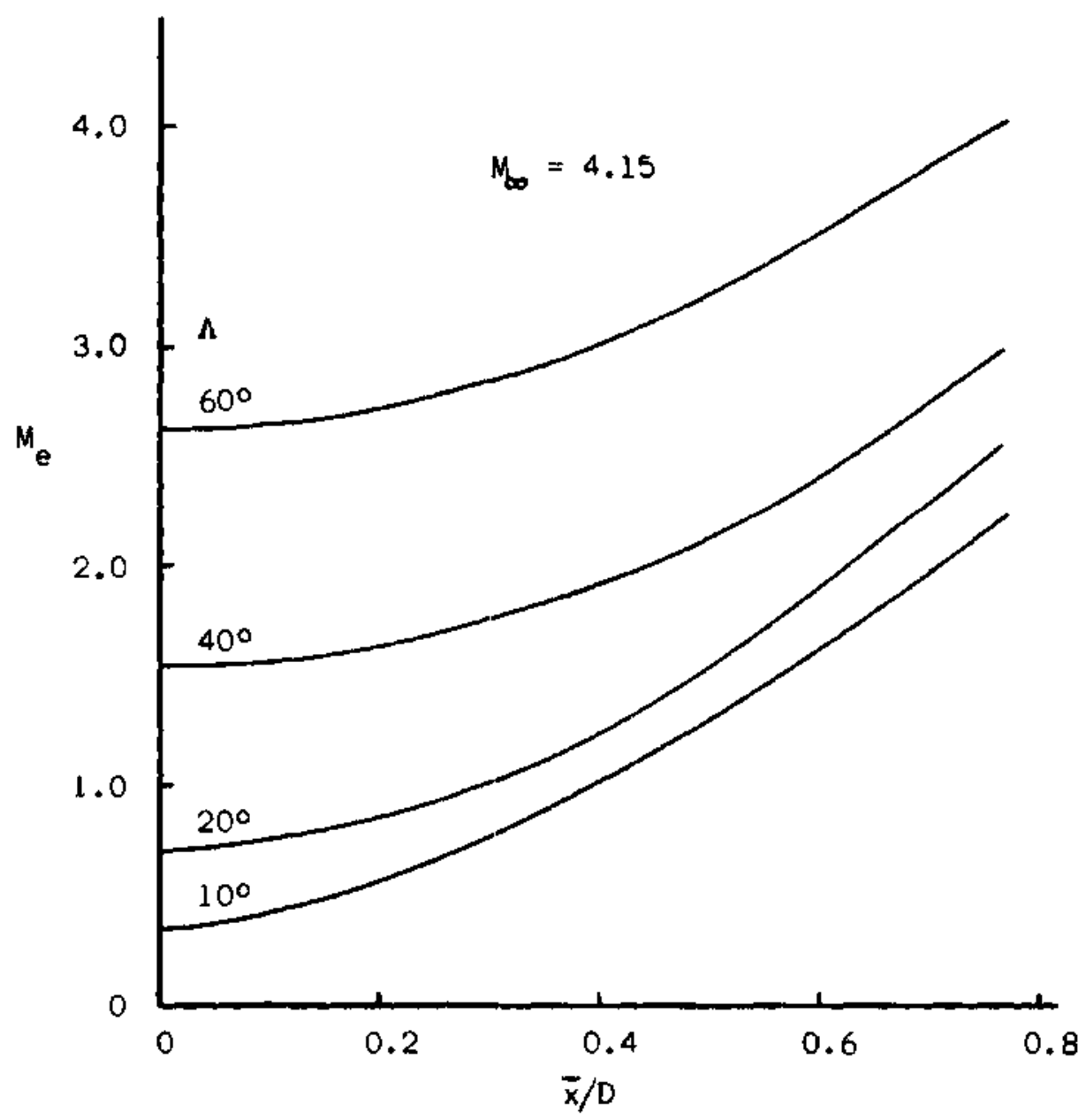

Figure 3. Local Mach Number Distribution for a Yawed Infinite Cylinder. 




Figure 4. Distance Along the Streamiine Coordinate for a Yawed Infinite Cylinder. 


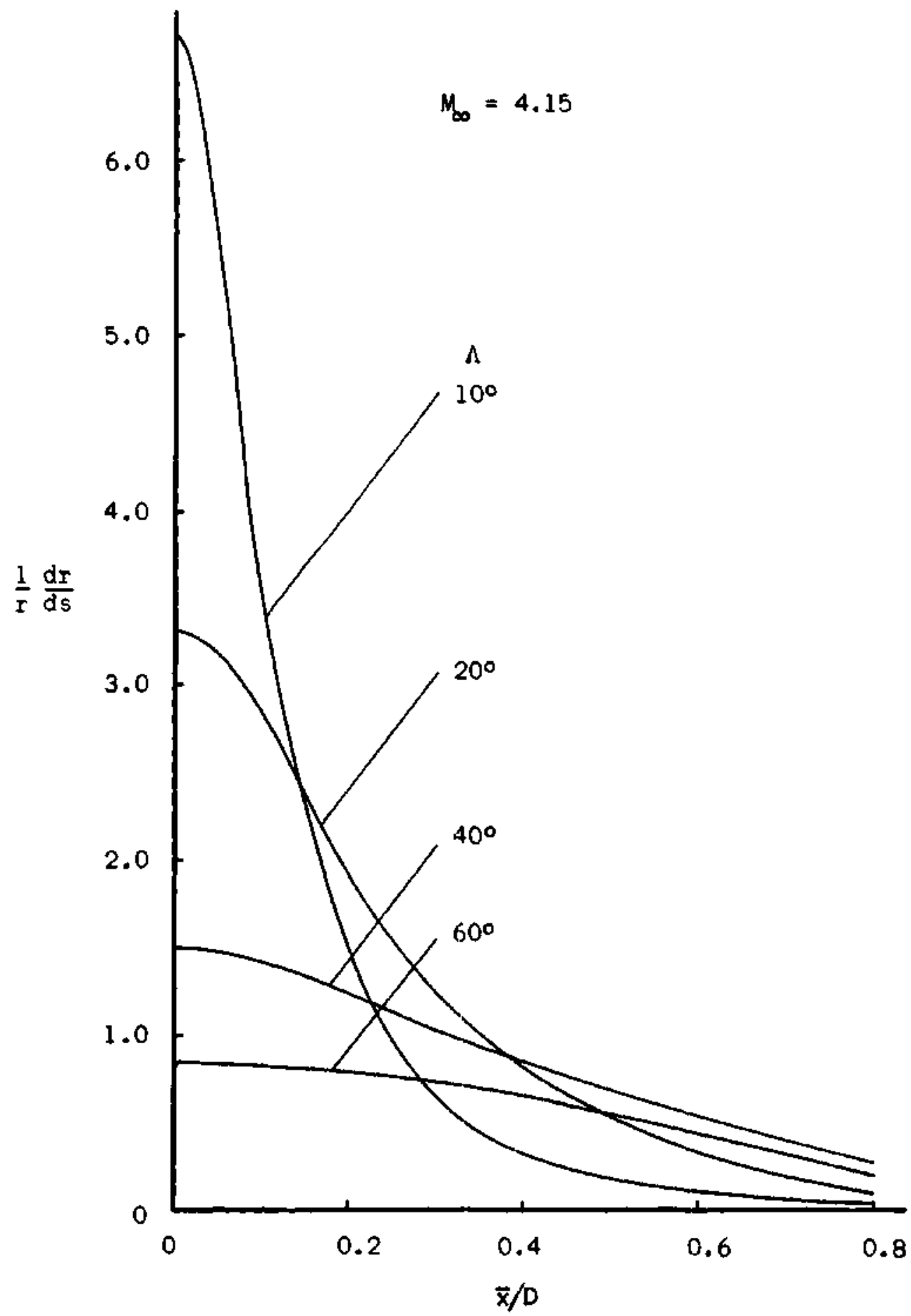

Figure 5. Streamline Divergence for a Yawed Infinite Cylinder. 




Figure 6. Inviscid Streamline Curvature Parameter for a Yawed Infinite Cylinder. 


$$
\frac{d H_{i}}{d \bar{s}}=\frac{\left(H_{i}^{2}-1\right) H_{i} C}{f}-0.011 \frac{\left(H_{i}-1\right)^{2}\left(H_{i}^{2}-1\right)\left(H_{i}+1\right)}{H_{i}^{2} f^{0.789}}\left(\frac{T_{e}}{I_{0}}\right)^{3} \frac{T_{0}}{I_{I}} R_{0} M_{e}
$$

with

$$
C=0.123 e^{-1.561 H_{i}} R_{o} M_{e} \frac{T_{e}}{I_{I}}\left(\frac{T_{e}}{T_{0}}\right)^{3}\left(\frac{\mu_{I}}{\mu_{0}}\right)^{0.268}
$$

and where $\frac{1}{r} \frac{d r}{d s}$ is a constant. On the stagnation line the cross-flow is identically zero so that the small cross-flow assumption is certainly valid in this case.

On the stagnation line of an infinite cylinder one notes from physical considerations that the values of $f$ (or $\theta_{11}$ ) and $H_{i}$ are invariant with distance $\bar{s}$ along the stagnation streamline. The general method for obtaining solutions is to set $\mathrm{dH}_{i} / \mathrm{d} \bar{s}$ and $\mathrm{df} / \mathrm{d} \bar{s}$ of equations 79 and 80 equal to zero and solve the two algebraic equations simultaneously for $H_{i}$ and $f$ using an iterative technique. In the present case, solutions were obtained in a manner similar to the integration technique applied over the forward face of the cylinder, that is, by integrating equations 79 and 80 along the stagnation streamline until $H_{i}$ and $f$ became constant. The initial conditions for these solutions were obtained by first integrating equation 79 for a few steps down stream with the initial value of $f$ equal to zero and $H_{i}$ held constant, then integrating equations 79 and 80 simultaneously along the streamline until the stationary solution was obtained.

Solutions to equations 79 and 80 were obtained on a Burroughs $B 5500$ digital computer employing a Runge-Kutta integration technjque. The 
Sutherland viscosity law was employed in the form

$$
\frac{\mu_{r}}{\mu_{0}}=\left(\frac{T_{r}}{T_{0}}\right)^{3 / 2} \quad\left(\frac{1+198 / T_{0}}{T_{r} / T_{0}+198 / T_{0}}\right)
$$

and the reference temperature $I_{r}$ was taken equal to the surface temper ature $T_{w}$ for the present moderate heat transfer case. This choice is discussed in Appendix E where the method of Sasman and Cresci is compared with two-dimensional experimental data for a flat plate and a favorable pressure gradient example. The wall temperature ratio $\mathrm{T}_{W} / \mathrm{T}_{0}$ was taken equal to 0.85 for all yaw angles. This value is representative of the data of reference 22. The boundary layer parameters $\theta_{1.1} / D_{,}{ }_{1}^{*} / D$, and $\mathrm{C}_{\mathrm{f}_{1} / 2}$ were computed from $\mathrm{f}$ and $\mathrm{H}_{i}$ using the relations of Chapter III. Typical results for a free stream Reynolds number, $R=P_{\infty} V_{\infty} D / \mu_{\infty}$, of $2 \times 10^{6}$ are presented in Figure 7 as a function of $A$. Of interest in this figure is the variation of the incompressible form factor $\mathrm{H}_{i}$ with yaw angle. For the low yaw angles, where the streamline divergence is greatest, $H_{i}$ takes on a significantly higher value than for the high yaw angles. Thus a computation method employing a constant $H_{i}$ based on flat plate data, say $1_{0} 3$, could be in substantial error even on the stagnation line where there is no streamwise pressure gradient. The use of the method of Sasman and Cresci (33) gives added flexibility in relating the assumed velocity profile closely to the flow under consideration through $\mathrm{H}_{1}$ 。

The heat transfer was computed making' use of Reynolds analogy in the form 


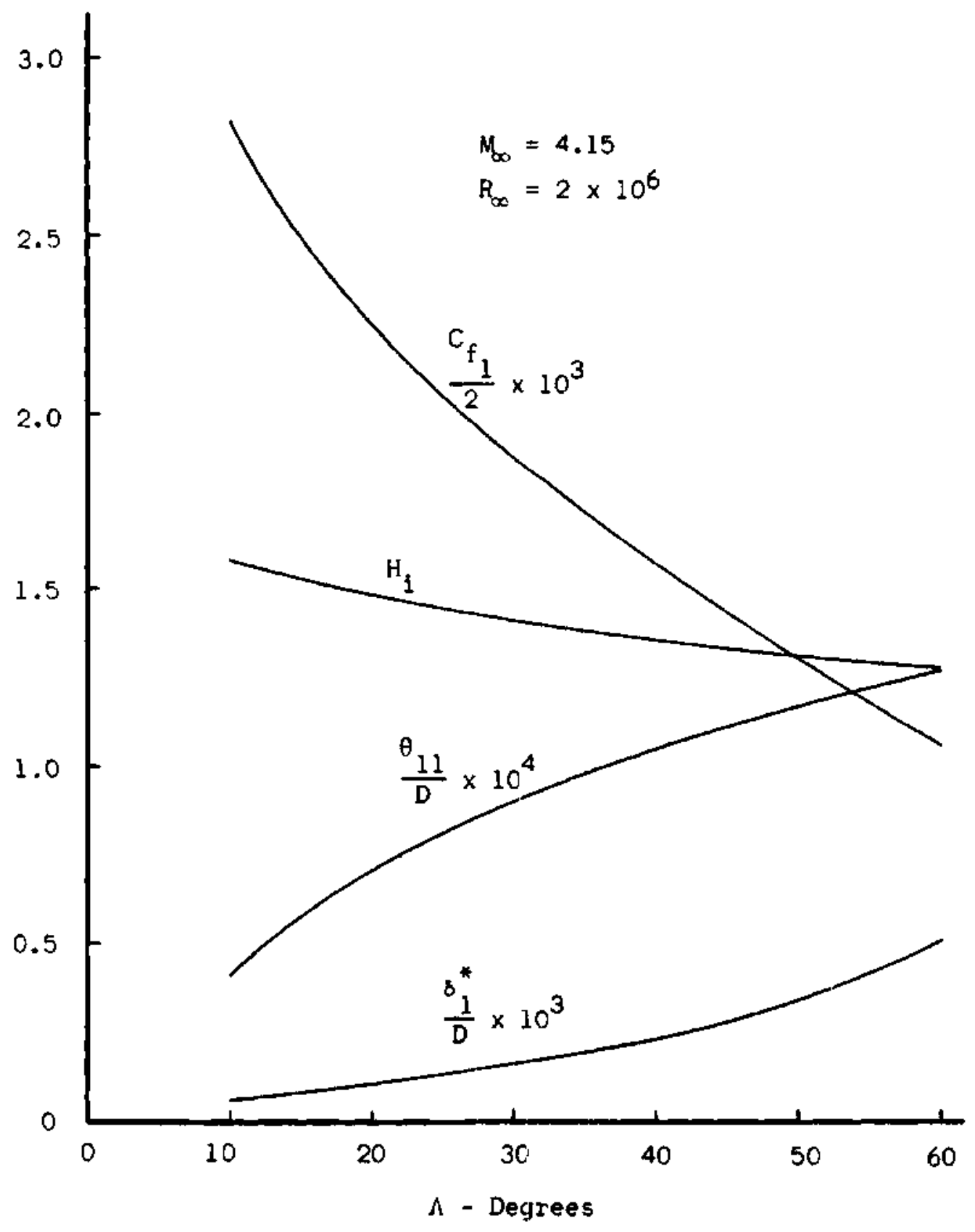

Figure 7. Computed Boundary Layer Parameters on the Stagnation Line. 


$$
s t \equiv \frac{q_{w}}{\rho_{e} u_{e} c_{p}\left(T_{a w}-T_{w}\right)}=p r^{-2 / 3} \frac{c_{f_{1}}}{2}
$$

where $S t$ is the Stanton number and $T_{\text {aw }}$ is the adiabatic wall temperature. Here $\operatorname{Pr}^{-2 / 3}$ is an empirical correction often used to account for Prandtl number not equal one. The Nusselt number was computed making use of equation 82 as

$$
N u \equiv \frac{q_{w} D}{k_{\infty}\left(T_{a w}-T_{w}\right)}=\operatorname{PrSt} R_{0} M_{e}\left(\frac{T_{e}}{T_{0}}\right)^{3} \frac{\mu_{0}}{\mu_{\infty}}
$$

where the Prandt 1 number is assumed constant and $Y=1.4$.

The heat transfer results are compared with the data of Beckwith and Gallagher (22) in Figure 8 as a function of Reynolds number. Good agreement of the theory with experiment is noted, and all the data appear to agree with the fully turbulent trends over the Reynolds number range as predicted by the present method except the $\Lambda=10^{\circ}$ case at low $R_{\infty^{*}}$ In this case transition to turbulent flow appears to be occurring for Reynolds number less than $2 \times 10^{6}$. Also shown are the theoretical calculations given by Beckwith and Gallagher. Comparison of these results with the present theory serve to corroborate the aforementioned importance of the streamline divergence effect on the velocity profiles as measured by $H_{i}$. The method of Beckwith and Gallagher, which is based on constant power velocity profiles and a Blasius friction law, differs appreciably from the present calculations for the low sweep angles.

Figure 9 presents the variation of stagnation line heat transfer: with yaw angle. Comparison is made with the data and theory of reference 


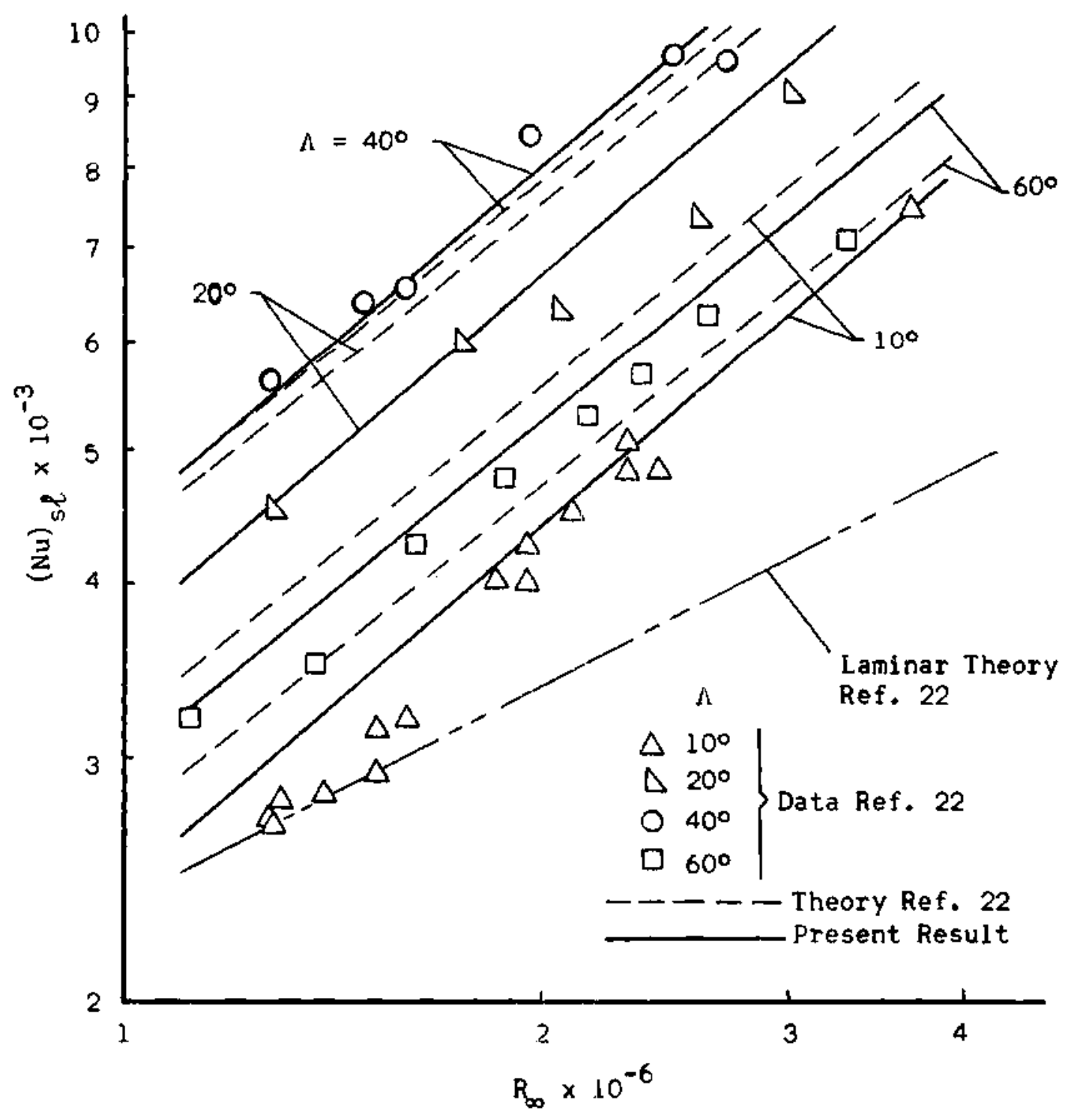

Figure 8. Stagnation Line Heat Transfer - Comparison with Experiment, $M_{\infty}=4.15$. 


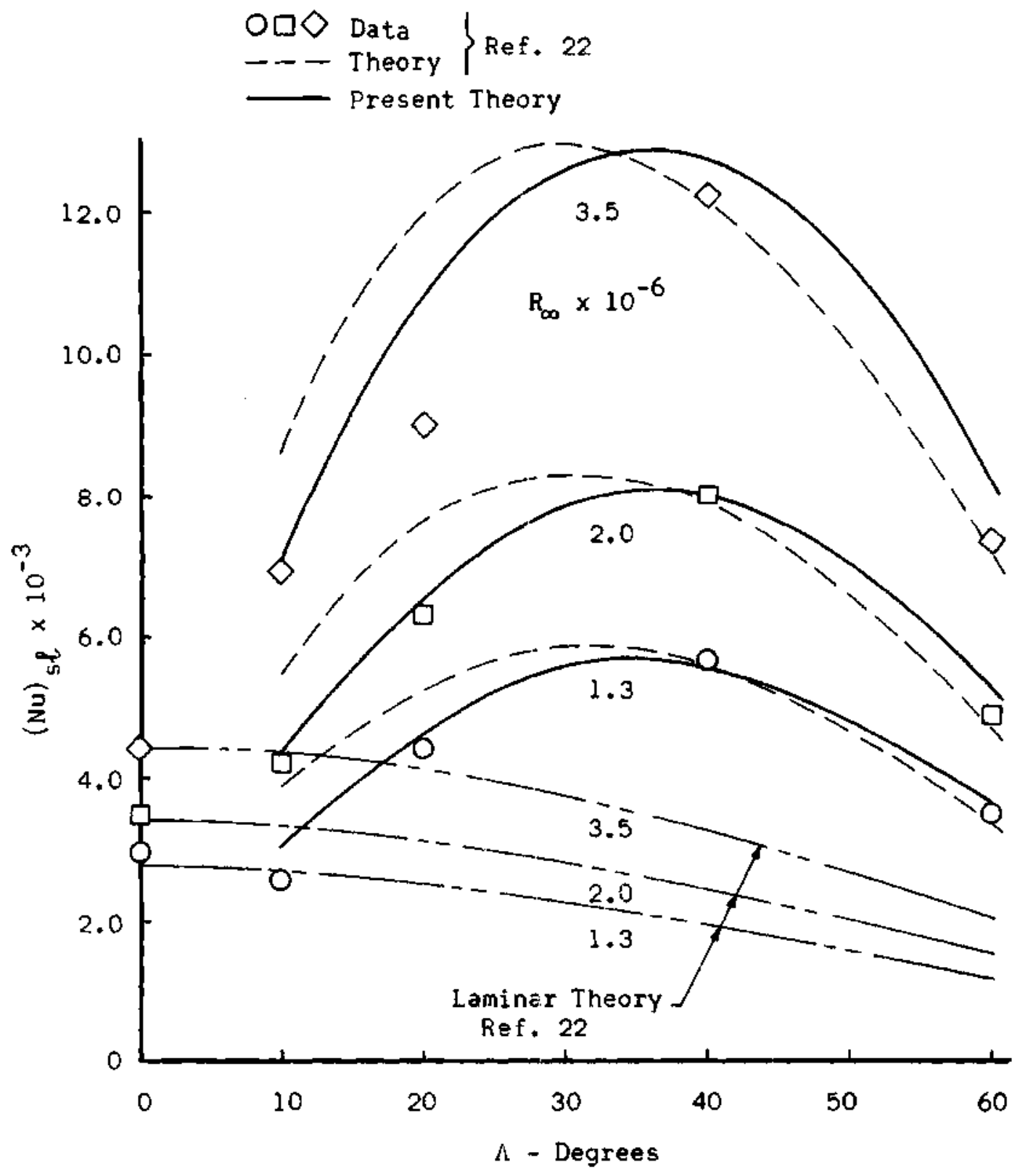

Figure 9. Stagnation Line Heat Transfer as a Function of Yaw Angle, $M_{\infty}=4.15$. 
22 for three Reynolds numbers. A peak in Nusselt number is noted for $\Lambda$ of approximately 35 degrees consistent with the trend of the experimental data.

\section{Streamwise Flow Solutions}

In this section solutions for the streamwise flow along a streamline path over the forward portion of the cylinder are presented. The governing equations are equations 62 and 63 . Integration of these equations was accomplished numerically just as for the stagnation line solution. The integration was started at a point near the stagnation line where initial values for $H_{i}$ and $f$ were assumed equal to those on the stagnation line as computed in the last section. The results of these calculations are presented in Figures 10 through 14 as a function of the chord-wise distance from the stagnation line $\bar{x} / D$.

The variation of the incompressible form factor $H_{i}$ is given in Figure 10 for yaw angles of 10, 20,40, and 60 degrees. The Reynolds numbers associated with each of these angles were selected to correspond to conditions tested by Beckwith and Gallagher in reference 22 . The form factor is noted to decrease rapidly away from the stagnation line, in agreement with the favorable streamwise pressure gradient (Figure 3). The corresponding thickness functions, $\theta_{11}$ and $\delta_{1}^{*}$, and the skin friction coefficient $C_{f_{1}}$ are given in $F$ igures 11,12 , and 13 , respectively. The heat transfer was computed assuming Reynolds analogy of the form given in equation 82 . This relation is noted to be strictly valid for zero pressure gradient flows over isothermal surfaces with the Ptandtl 


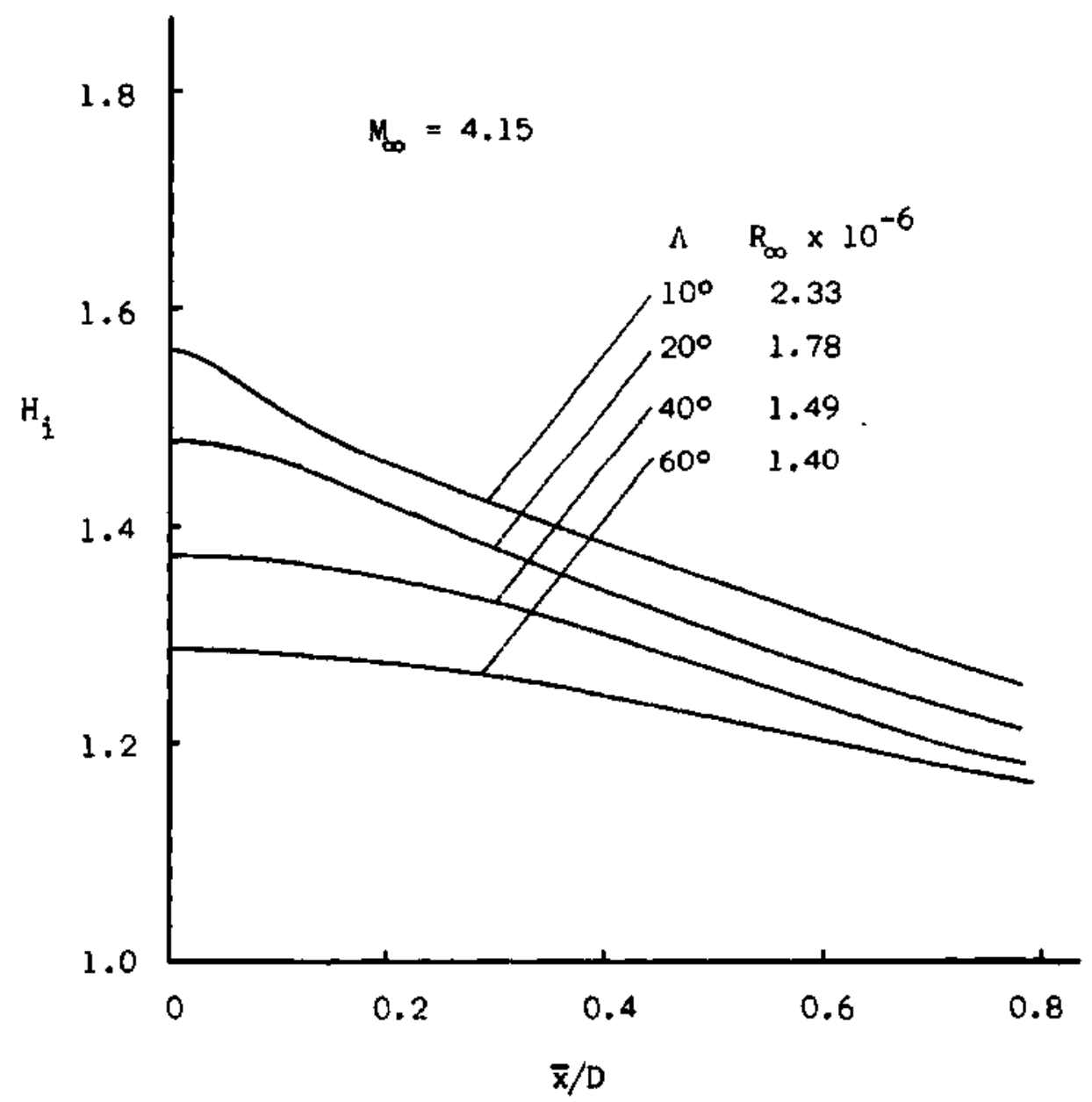

Figure 10. Computed Variation of $\mathrm{H}_{i}$ for
the Yawed Cylinder. 


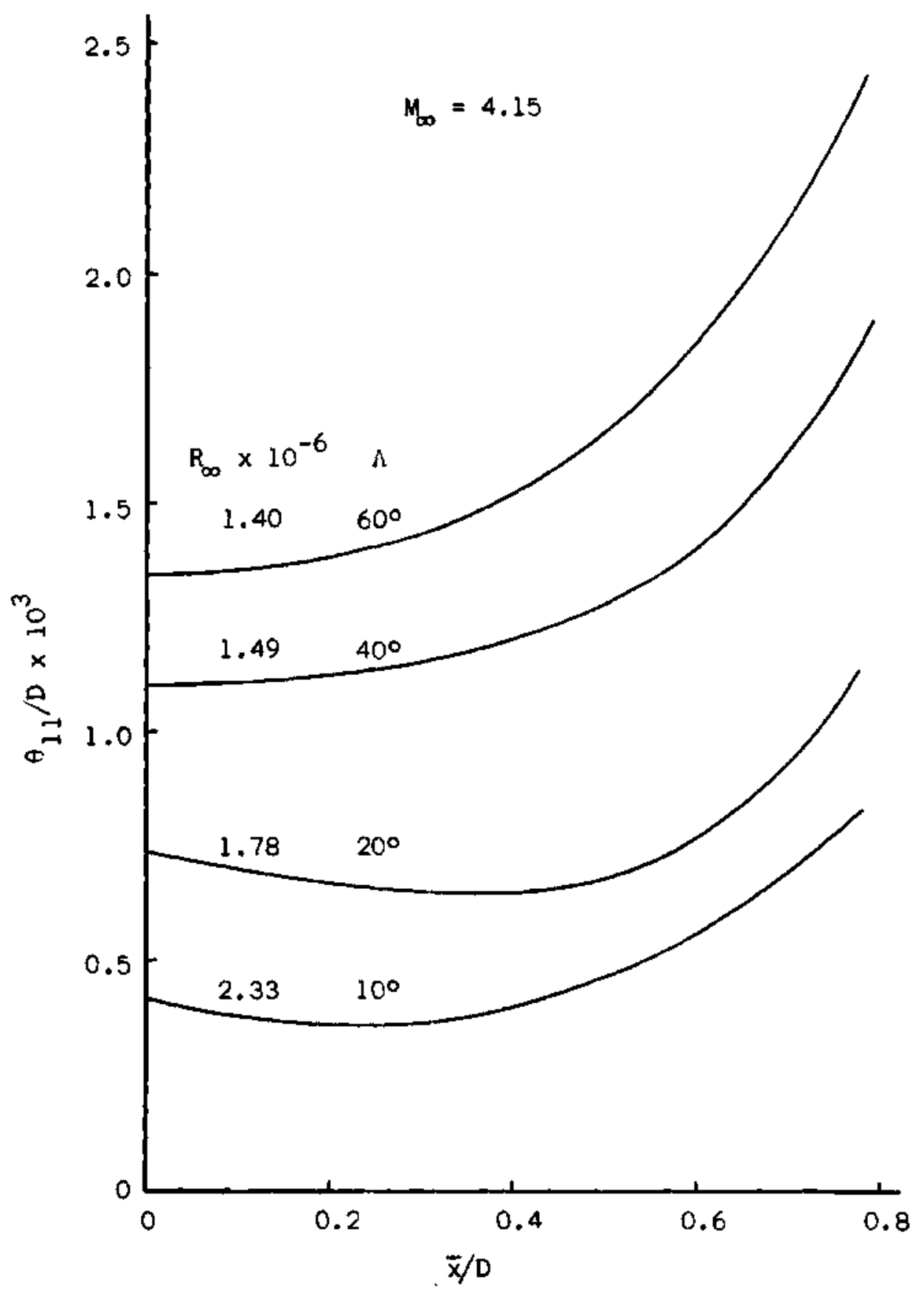

Figure 11. Computed Momentum Thickness for the Yawed Cylinder. 


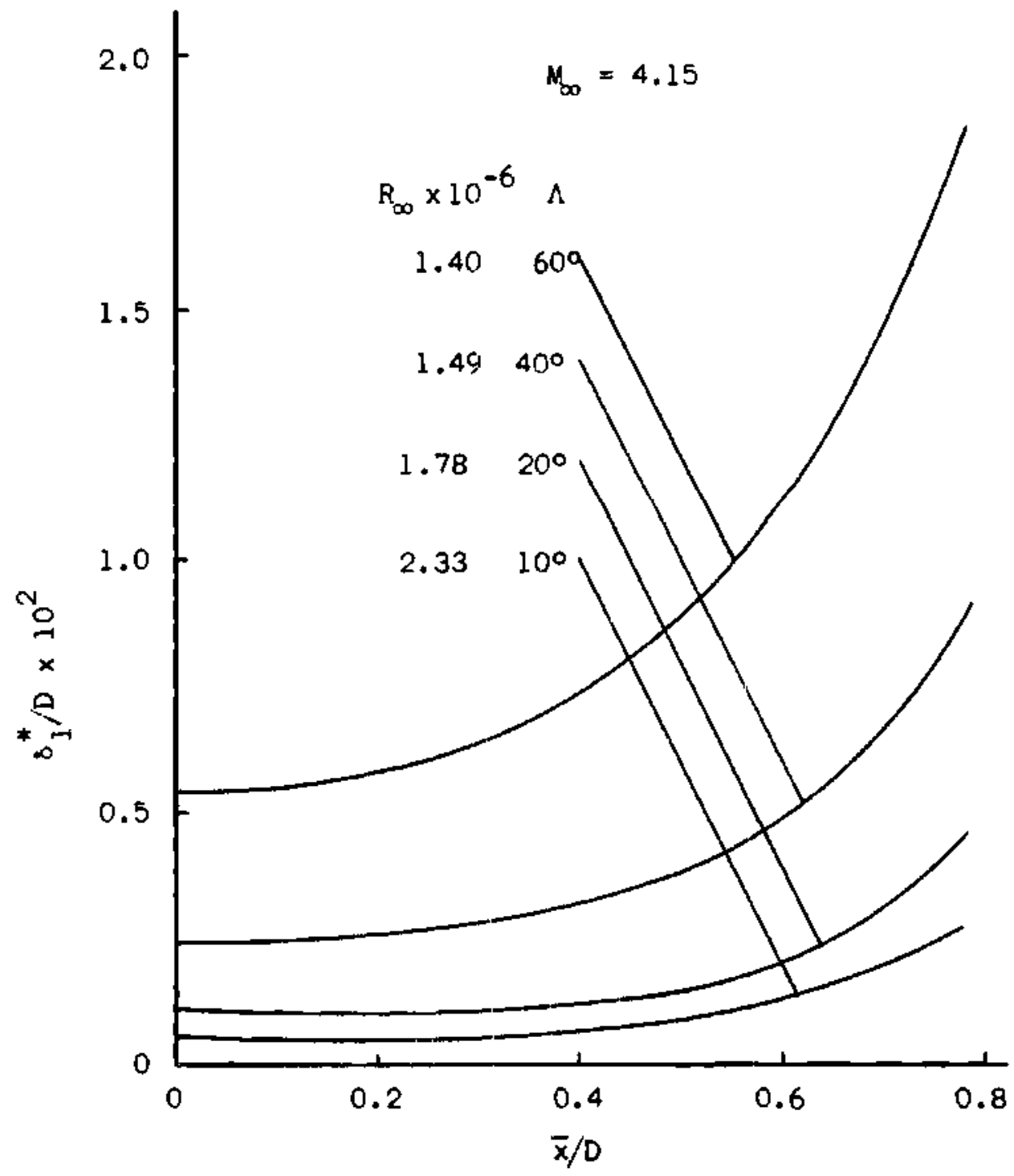

Figure 12. Computed Displacement Thickness for the Yawed Cylinder. 


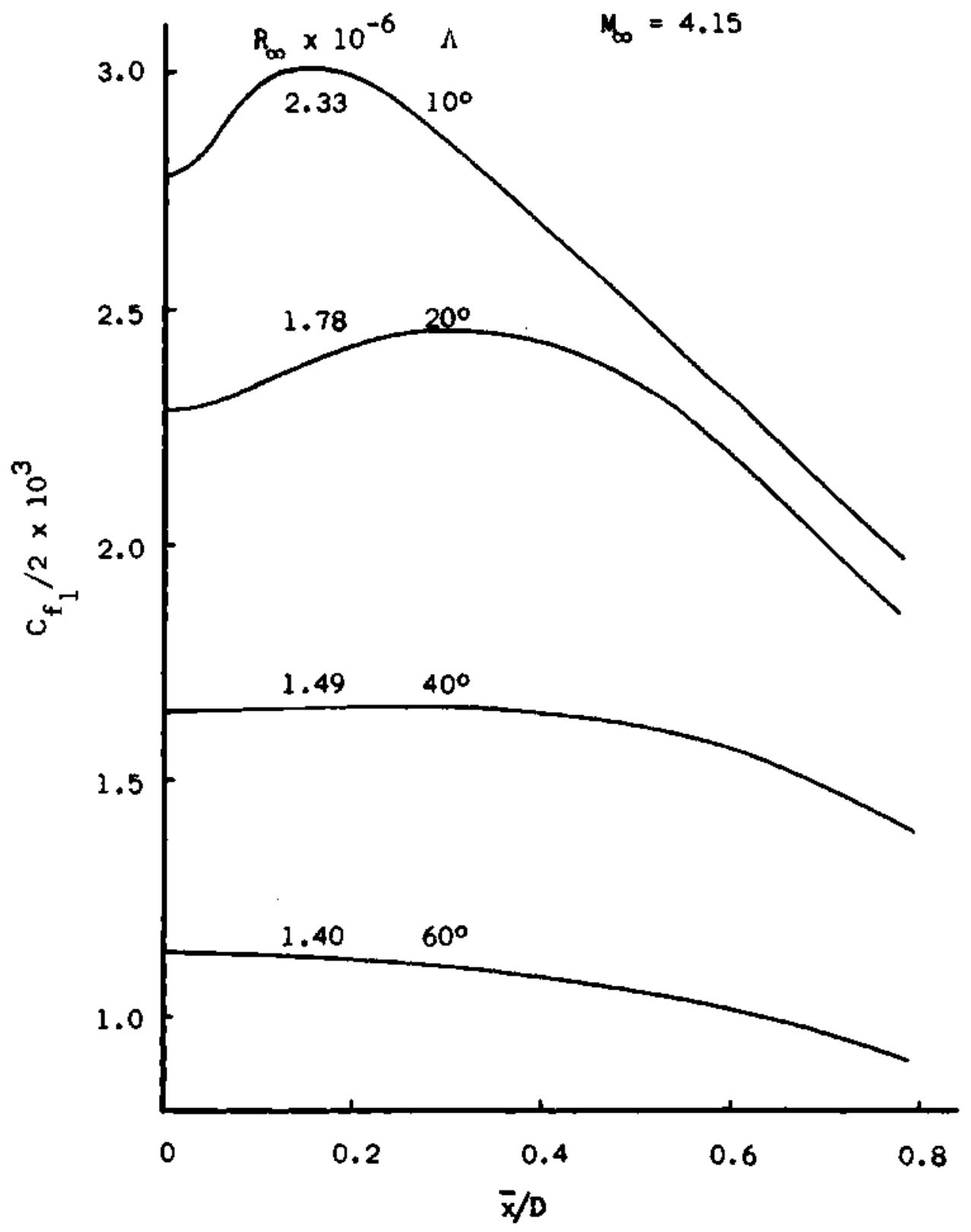

Figure 13. Computed Streamwise Skin Friction Coefficient for the Yawed Cylinder. 


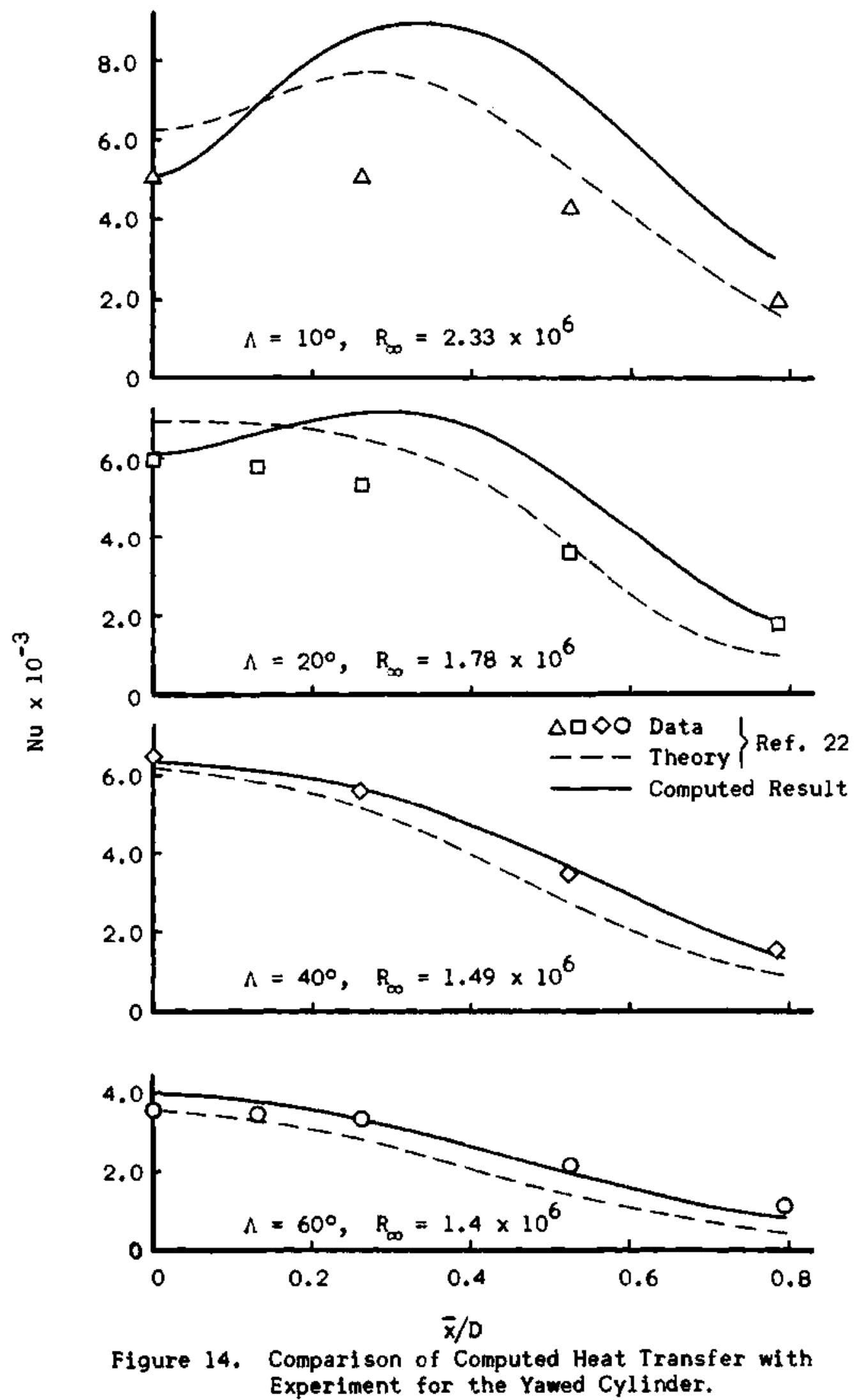


number equal to one. Although the expression has been applied to flows with pressure gradient by numerous workers, its applicability remains open to question. This point is discussed further in conjunction with the comparisons of the present theory with experiment.

The computed heat transfer in the form of Nusselt number is compared with the experimental data and theoretical results of Beckwith and Gallagher in Figure 14 for yaw angles of 10, 20, 40, and 60 degrees. The cross-flow will be shown in the next section to be small for all four yaw angles under the present flow conditions and, therefore, is expected to have little effect on the results. Good agreement between computed and experimental Nusselt number for yaw angles of 40 and 60 degrees is noted in this figure. In these cases the flow along an inviscid streamline develops under a moderate favorable pressure gradient. The use of Reynolds analogy is given support by the experimental work of Pasiuk et al. (42) who verified its application for a flat plate with moderatie heat transfer and with a mild favorable pressure gradient.

On the other hand examination of Figure 14 reveals that for the low yaw angles, 10 and 20 degrees, the computed Nusselt number is considerably higher than the data and reaches a peak in the vicinity of $\bar{x} / D$ equal to 0.3 . This trend is noted to be similar to theoretical turbulent boundary layer calculations for cylinders at zero yaw and blunt bodies as, for example, in references $22,31,43$, and 44 . Strongly suspect in the present calculation is the application of the flat plate Reynolds analogy (equation 82). At low yaw angles the flow along the streamline coordinate $\bar{s}$ away from the stagnation streamline develops much as the flow over a blunt body, that is, under the influence of a 
strong favorable streamwise pressure gradient. For example, the pressure gradient term $\frac{1}{M_{e}} \frac{d M_{e}}{d \bar{s}}$ is an order of magnitude greater for the 10 degree yaw case than for the 60 degree yaw case. Some confirmation of Reynolds analogy has been noted under such conditions when the cooling is high as discussed by Cresci et al. (43). In the present application, however, the cooling is moderate, $\mathrm{T}_{w} / \mathrm{T}_{0}=0.85$, thus leaving quite unresolved the question of applicability of Reynolds analogy. A detailed discussion of this anomaly is beyond the scope of the present work. The reader is directed to references 31,43 , and 45 for further literature in this area.

\section{Cross-Flow Solutions}

The cross-flow momentum integral equation (equation 64) was programmed along with the streamwise equations for numerical integration. Solutions were obtained, dependent on the streamwise solution, utilizing a Runge-Kutta integration method. The initial condition for this integration was given by $A$ equal zero at the starting point near the stagnation line.

The basic features of the solution are illustrated in Figure 15 where the cross-flow parameter A corresponding to the streamwise solutions of the preceding section is plotted versus $\bar{x} / D$. The functional dependence of these solutions on the streamline curvature parameter $k$ as given in Figure 6 is apparent. The maximum value of $A$ occurs near: the stagnation line for the lower yaw angles and moves toward the shoulder of the cylinder as $\Lambda$ becomes larger. These solutions are examined in terms of the criteria for small cross-flow in the next figure. 


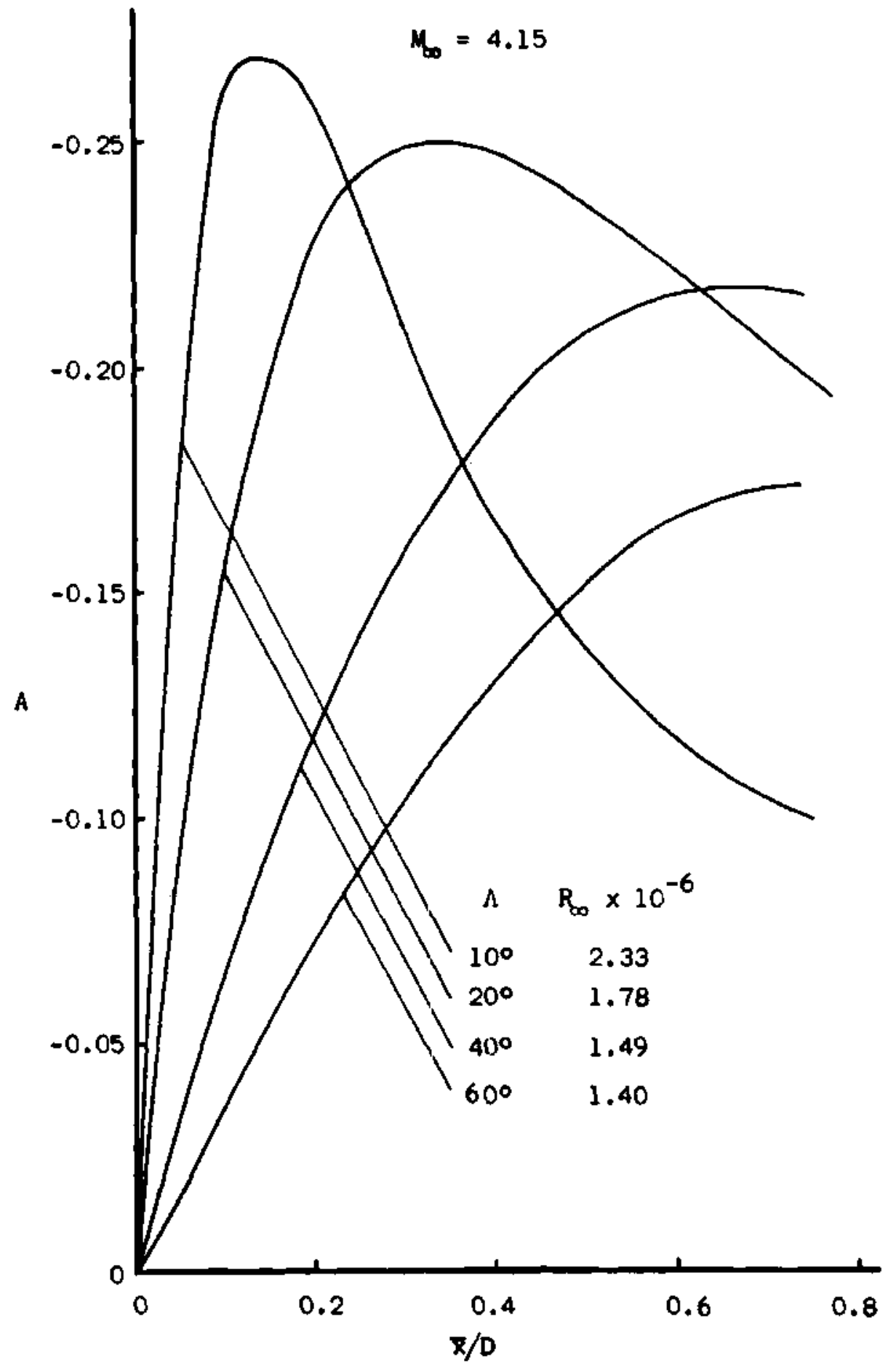

Figure 15. Cross-Flow Solutions - Yawed Cylinder Example. 
Recalling that $(v / u)^{2} \ll 1$ is required for the cross-flow to be small, the maximum value of this ratio (related to $A$ by equations 55 and 58) is plotted in Figure 16. Here one notes that the cross-flow grows most rapidly as the flow moves away from the stagnation line for the low yaw cases but reaches the greatest magnitude in the vicinity of the shoulder at the higher yaw angles. A comparison of the magnitude of this parameter with the laminar results of Beckwith (29) for a yawed cylinder indicates that the turbulent cross-flow is appreciably less than for a similar laminar flow. This result is to be expected in view of the previous discussion in Chapter II.

It is further noted that for the flow conditions considered here the velocity ratio $(\mathrm{v} / \mathrm{u})_{\max }^{2}$ is less than 0.1 , except for regions near the shoulder at high yaw angles. Thus, the effects of cross-flow on the skin friction and heat transfer are expected to be small. This is verified by the good agreement of the streamwise solution with heat transfer data for yaw angles of 40 and 60 degrees (Eigure 14). Also in view of this agreement at high yaw angles, the lesser cross-flow at yaw angles of 10 and 20 degrees could not account for the gross overprediction of Nusselt number by the streamwise solution as given in Figure 14. In this sense the skin friction and thickness functions of Figures 11, 12, and 13 perhaps are correctly computed by the Sasman and Cresci method as applied here, with the error in Nusselt number at low yaw angles being attributed to Rey.s nolds analogy. Of course only detailed experimentation can confirm this. In the remaining portion of this chapter some of the basic features of the cross-flow solution will be discussed with regard to the effects of the velocity profile assumption, wall cooling, and streamwise form factor 


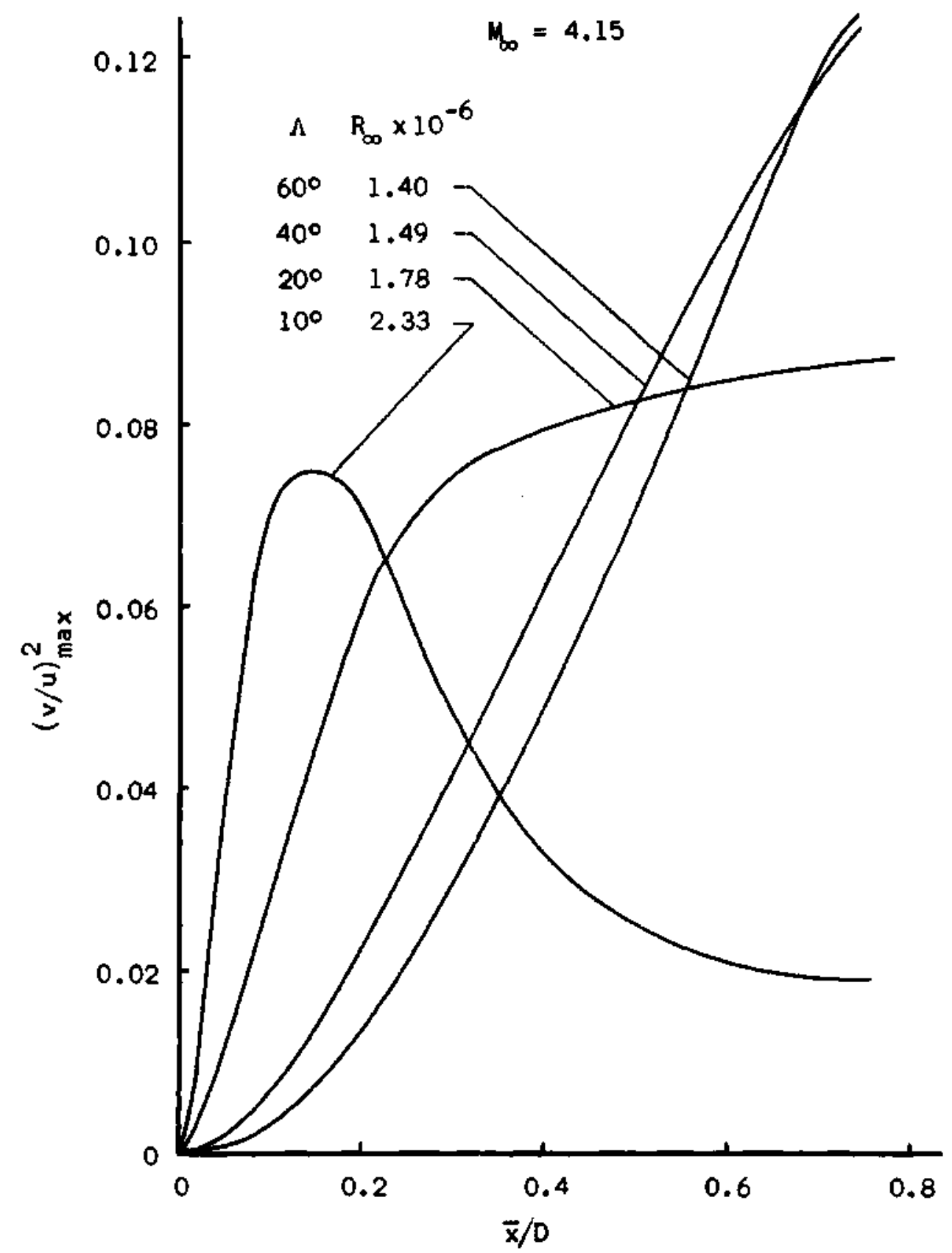

Figure 16. Maximum Value of Cross-Flow to Streamwise Velocity Ratio. 
parameter $H_{i}$. In order to establish a consistent frame of reference for this discussion, the yawed cylinder example for a Reynolds number of $2 \times 10^{6}$ is considered, and the solution is given in form of the ratio of the components of skin friction $\mathrm{C}_{f_{2}} / \mathrm{C}_{f_{1}}$ (equal to $\tan$ a). Figure 17 presents a plot of this ratio versus $\bar{x} / D$ for yaw angles of 10,20 , 40 , and 60 degrees. Ihis solution incorporates the "basic" parabolic cross-flow velocity profile assumption (equation 50 ) which is believed to be a reasonable approximation for the present case where the strearn wise pressure gradient is favorable in all cases. Hall's experiments (24) tend to support this statement as discussed in Chapter III.

Typical compressible velocity profiles are shown in Figure 18 both in the streamwise and in the cross-flow directions. These profiles were computed from the assumed relations in the transformed plane, equa. tions 28 and 50, for the 60 degree yaw case where the normal coordinate $Z / \Delta$ was transposed to the compressible plane using the results of Appendix $F$. The general shape of the parabolic cross-flow velocity rela: tion is illustrated in this figure and the effect of the favorable pres. sure gradient on the assumed streamwise velocity profile is evidenced by the "filling" of the profile near the surface as $\bar{x} / D$ increases. It was shown in Chapter III that the maximum value of $v / u$ is equal to $\tan a$ and occurs in the limit as the surface is approached. Of course here both $u$ and $v$ approach zero also. Another parameter of interest is the maximum value the cross-flow velocity attains as compared to the local inviscid flow velocity. Appendix G presents an expres= sion for this parameter, $\left(v / u_{e}\right)$ max as a function of $\tan a$ and the streamwise form factor $H_{i}$. Figure 19 gives the variation of $\left(v / u_{e}\right)_{\max }$ 


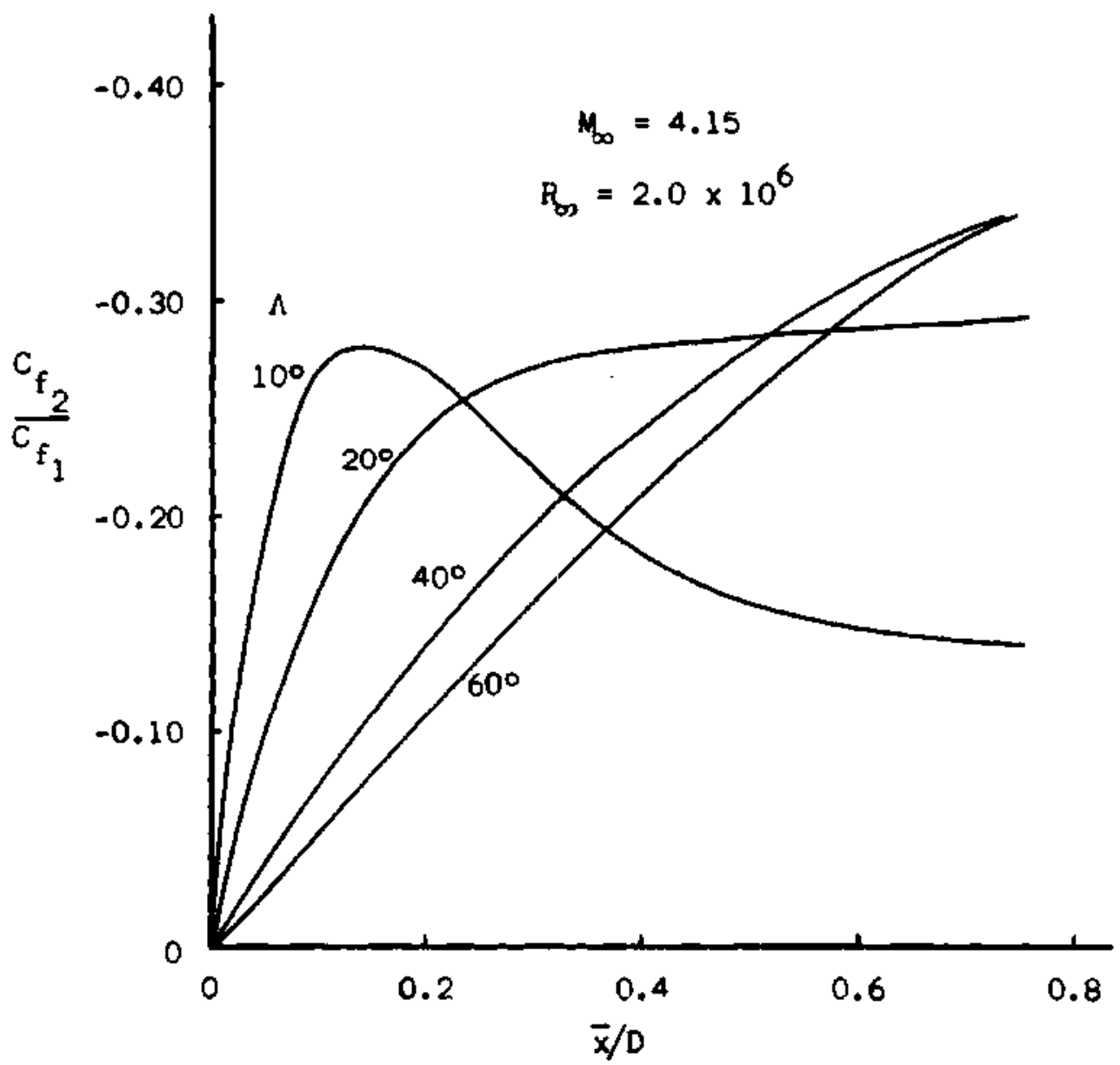

Figure 17. Ratio of Cross-Flow to Streamise Skin Friction Components. 


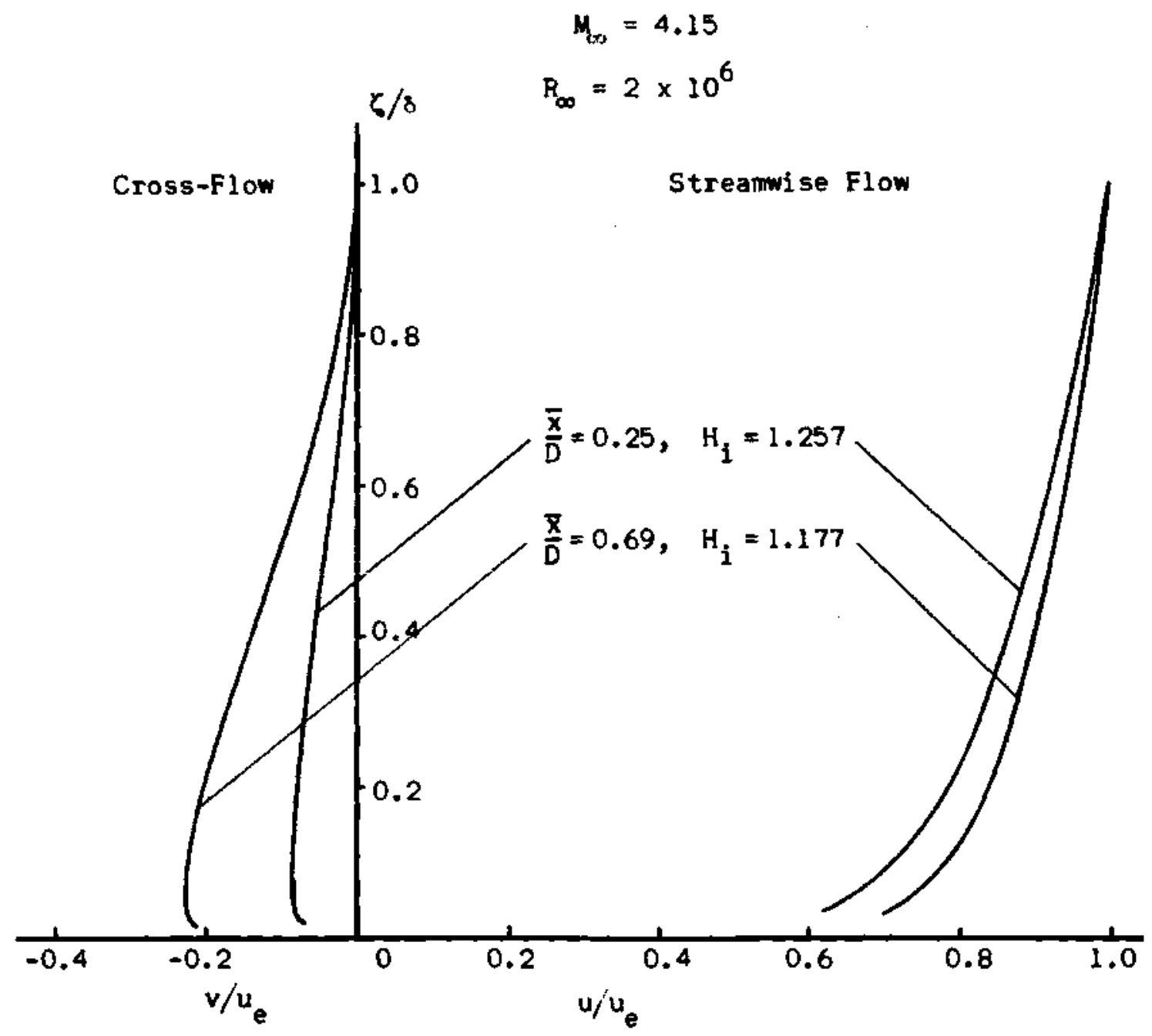

Figure 18. Typical Velocity Profiles for the Yawed Cyliñer Example, $h=60^{\circ}$. 


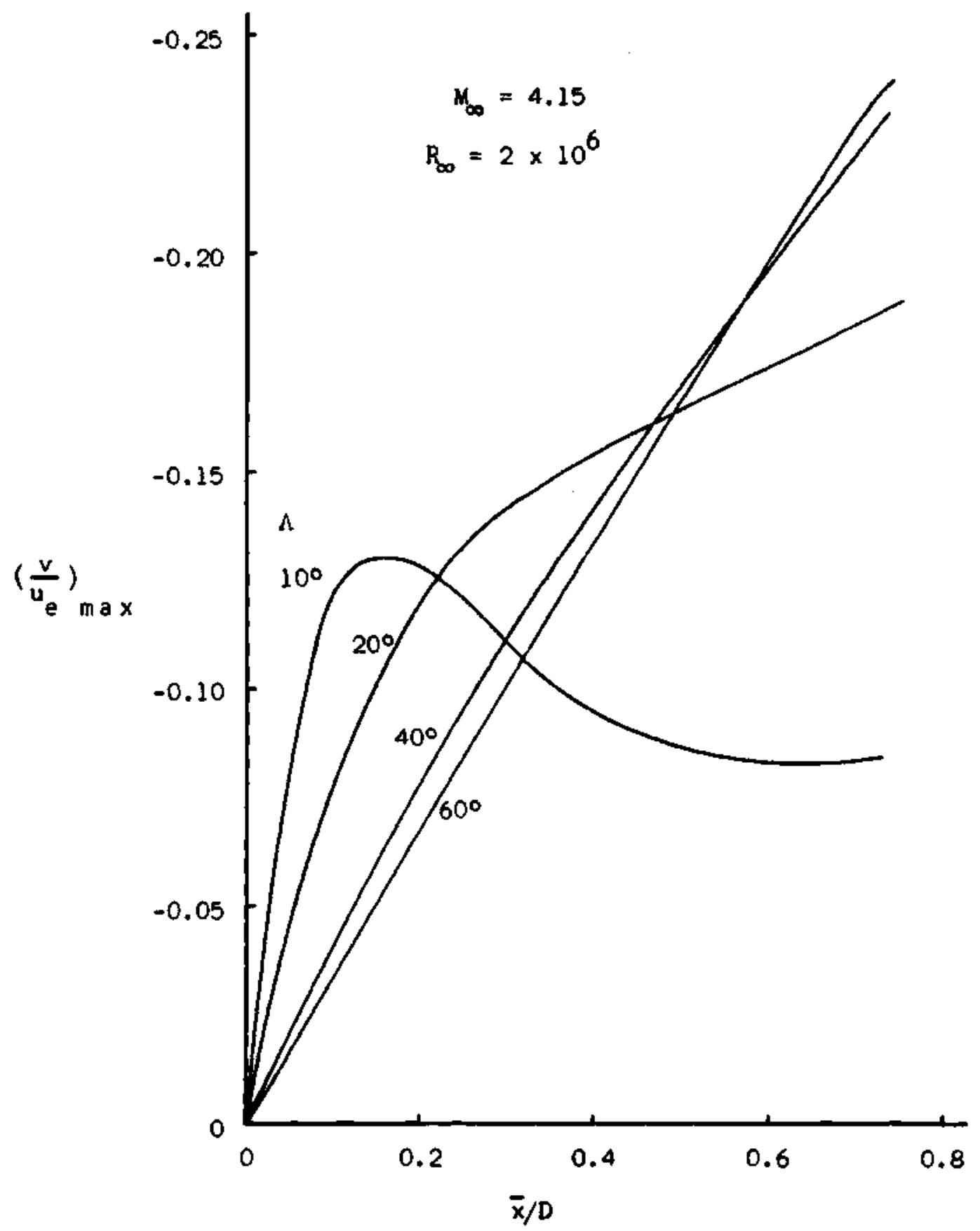

Figure 19. Maximum Cross-Flow Velocity for the Yawed Cylinder. 
for the yawed cylinder example. For profiles of the form assumed here (equation 54) coupling exists between the streamwise flow development and the cross-flow profiles, as is evidenced. by the influence of $H_{1}$ in the momentum integral equation (equation 51 and related equations in Appendix C) as well as by the functional relations of Appendix G. Thus a streamwise solution which takes into account the flow effect on the streamwise velocity profile, as does the Sasman and Cresci method, is desirable if the cross-flow is to be computed. Profile Assumption Effect

In view of the uncertainty associated with the assumed form o: the cross-flow velocity profile a measure of the effect this assumption has on the solution of the cross-flow momentum equation is of interest. This effect has been examined for the present yawed cylinder example by assuming two alternate profile relations of the same general form as the parabolic relation. In particular, the form of equation 54 is assumed

$$
\frac{V}{U_{e}}=\left(1-\frac{Z}{\Delta}\right)^{n} \frac{U}{U_{e}} \tan \bar{\alpha}
$$

where $n=2$ gives the parabolic relation employed in the previous solutions, and $n=1$ is denoted as the "linear" relation and $n=3$ as the "cubic" relation. The corresponding momentum integral equations are given in Appendix C. These equations were solved for the yawed cylinder, and comparisons of these results with the preceding results based on the parabolic profile are presented in the following figures for Reynolds number of $2 \times 10^{6}$ and yaw angles of 10 and 60 degrees. The resulting compressible velocity profile shapes are illustrated 
In Figure 20. These plots are typical profiles computed for the 60 degree yaw case employing the results of Appendix F. The difference in the three profile assumptions is mainly evidenced in the shift of the maximum velocity nearer to the surface as the exponent $n$ in equation 54 increases. The increase in magnitude of this velocity results from the solution of the momentum integral equation, i.e. $\tan a$, as is illustrated more clearly in the next two figures.

Figures 21 and 22 present the effects of the profile assumption on the cross-flow solution in the form of the ratio of normal to streamwise skin friction coefficients $C_{f_{2}} / C_{f_{1}}$ (equal to tan a) for yaw angles of 10 and 60 degrees. The cross-flow magnitude as measured by $\mathrm{C}_{\mathrm{f}_{2}} / \mathrm{C}_{\mathrm{f}_{1}}$ is seen to be directly proportional to the exponent $n$ in the assumed profile relation with the greatest value resulting from the cubic rela. tion and the least value for the linear relation. The profile assumption is noted to have a rather significant effect on the cross-flow solution. For example, the maximum value of $\mathrm{C}_{f_{2}} / \mathrm{C}_{f_{1}}$ resulting from the cubic relation is approximately 30 percent higher than the parabolic result for the 60 degree yaw case and about 25 percent; higher for 10 degrees yaw. The need for further experimental confirmation of the proper form for the cross flow velocity profile is apparent, particularly for flows where the streamwise pressure gradient is not always favorable as in the present case. Wall Cooling Effect

Increasing the cooling at the wall tends to thin the boundary layer and fill the streamwise velocity profile near the surface. In threedimensional layers this effect acts to retard the development of flows normal to the inviscid flow direction in view of the increased momentum in 


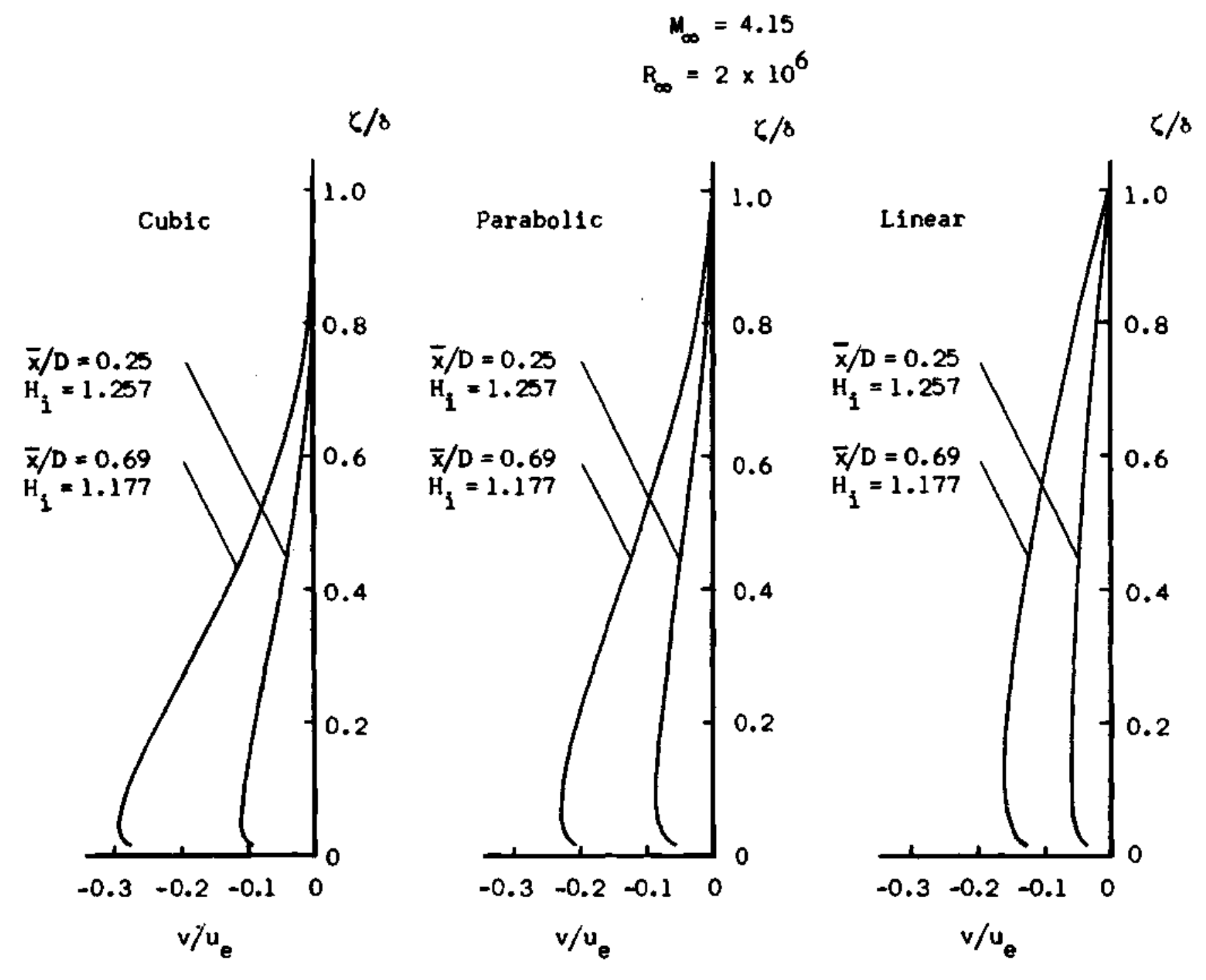

Figure 20. Typical Velocity Profiles for Three Assumed Shape Relations, $\Lambda=60^{\circ}$. 


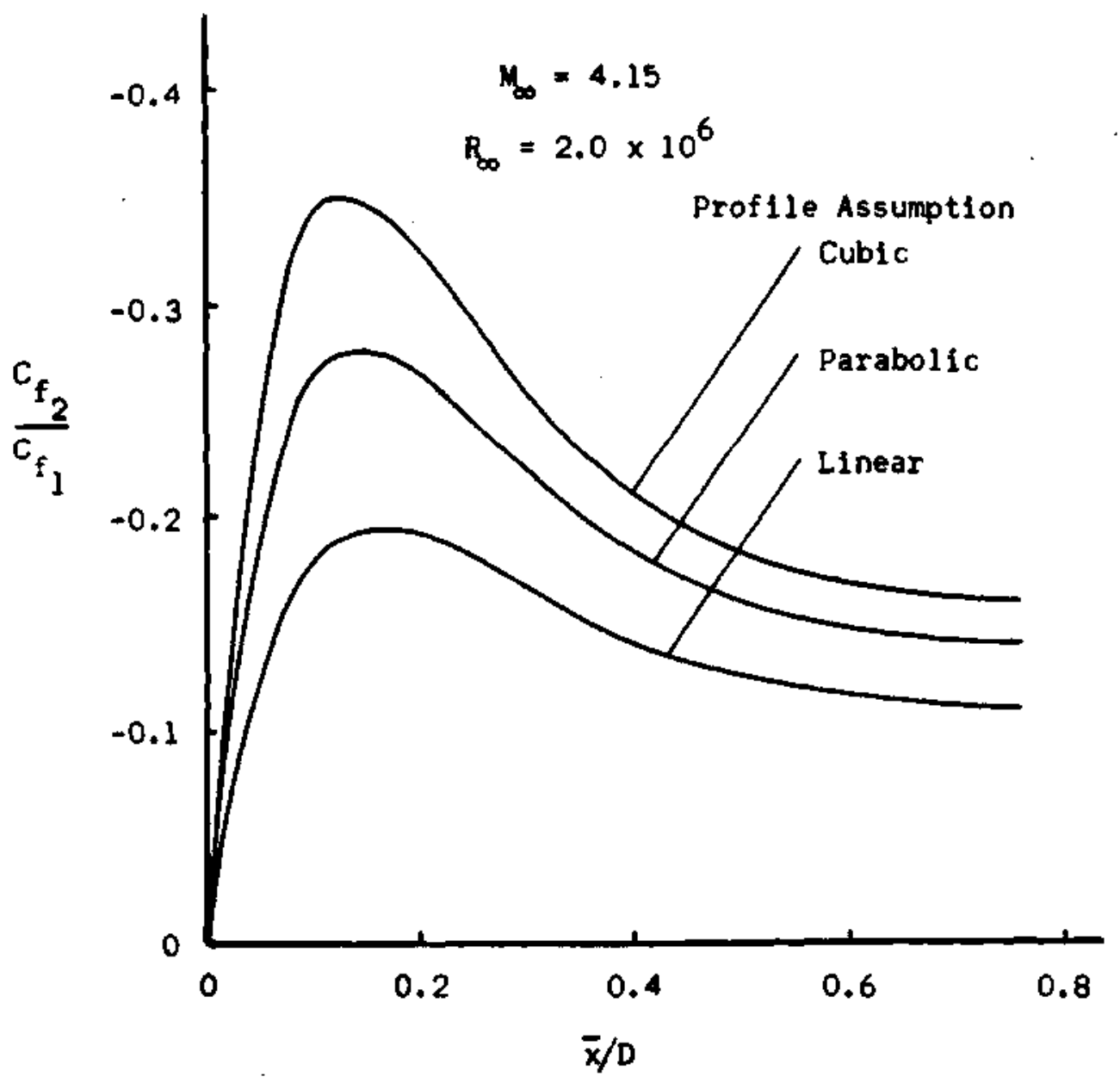

Figure 21. Effect of Velocity Profile Assumptions on the Cross-Flow Solution, $\Lambda=10^{\circ}$. 


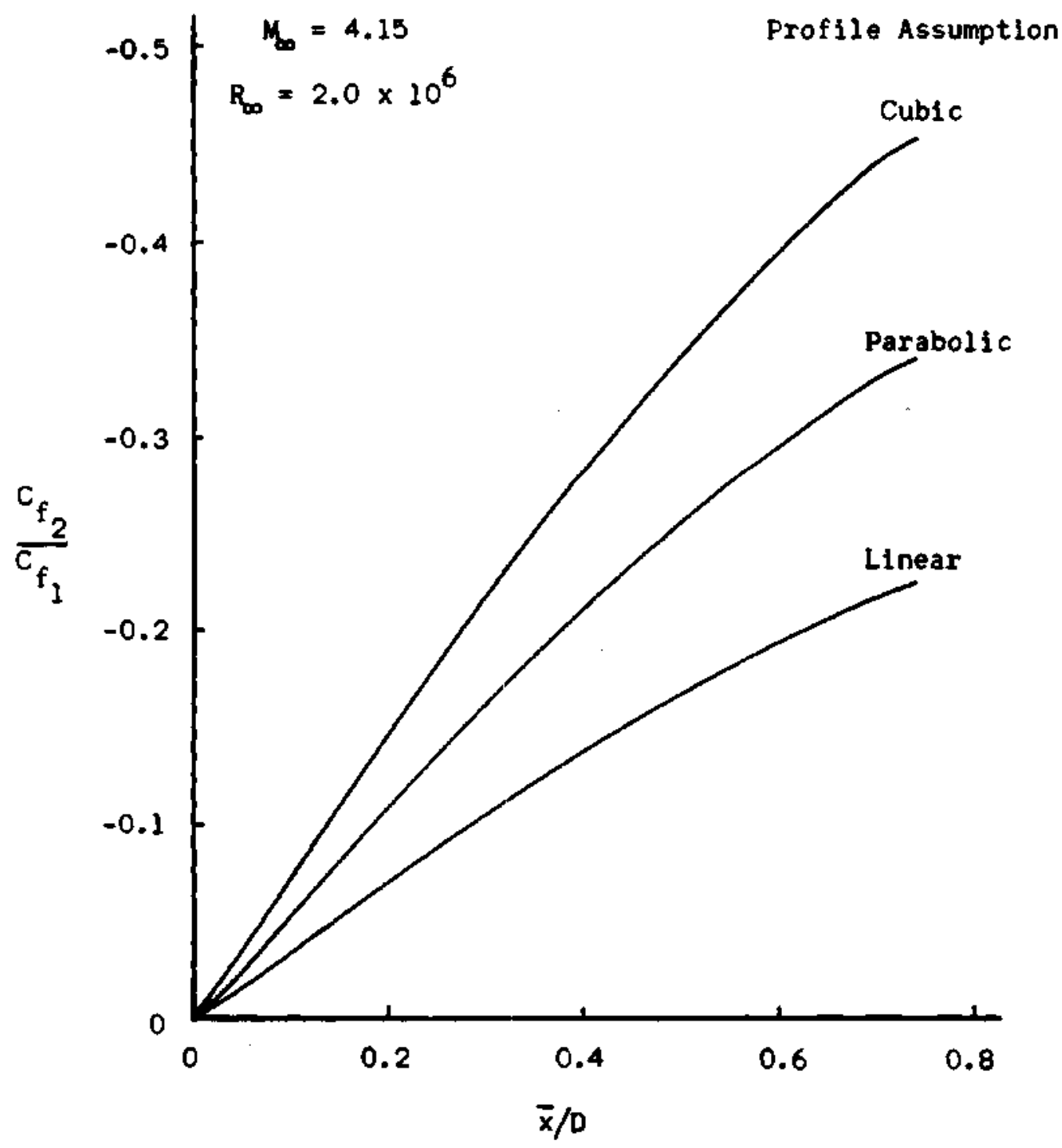

Figure 22. Effect of Velocity Profile Assumptions on the Cross-Flow Solution, $\Lambda=60^{\circ}$. 
the lower portions of the boundary layer. The degree to which additional cooling reduces the secondary flow for the yawed cylinder is illustrated in the following results.

Figures 23 and 24 present solutions to the cross-flow equation for yaw angles of 10 and 60 degrees where the wall cooling $I_{W} / T_{0}$ is considered as a parameter. The ratio $C_{f_{2}} / C_{f_{1}}$ is plotted versus $\bar{x} / D$ for $T_{W} / T_{0}$ equal $1.0,0.6$, and 0.2 in these figures One notes a decrease in $\mathrm{C}_{f_{2}} / \mathrm{C}_{f_{1}}$ of approximately 50 percent when the high cooling case $\left(\mathrm{T}_{W} / \mathrm{T}_{0}=0.2\right)$ is compared with the adiabatic wall case $\left(\mathrm{T}_{\mathrm{W}} / \mathrm{T}_{0}=1.0\right)$. Thus the range of flow conditions for which the small cross-flow assurnption is valid may be expected to be extended when the cooling of the surface is high as has been indicated by Vaglio-Laurin (21). $\underline{\text { i Effect }}$

In the foregoing discussions the close relationship between the cross-flow and the developing streamwise flow has been pointed out with regard to the dependence of the cross-flow solution upon the streamwise form factor parameter $H_{1}$. Since oftentimes two-dimensional computation methods are based on assumptions of constant $H_{i}$ (for example references 23 and 31 ) it is of interest to see how such an assumption might affect the cross-flow solution. A solution for the 60 degree yaw case with $H_{1}$ held constant at 1.3 is compared in Figure 25 with the variable $H_{1}$ solution as computed previously. The streamwise solution was obtained by solving equation 62 with $H_{i}$ held constant at 1.3 and using this result to solve the cross-flow equation. The qualitative effect of $\mathrm{H}_{i}$ is illustrated in Figure 25 where a sharp increase in cross-flow is apparent 


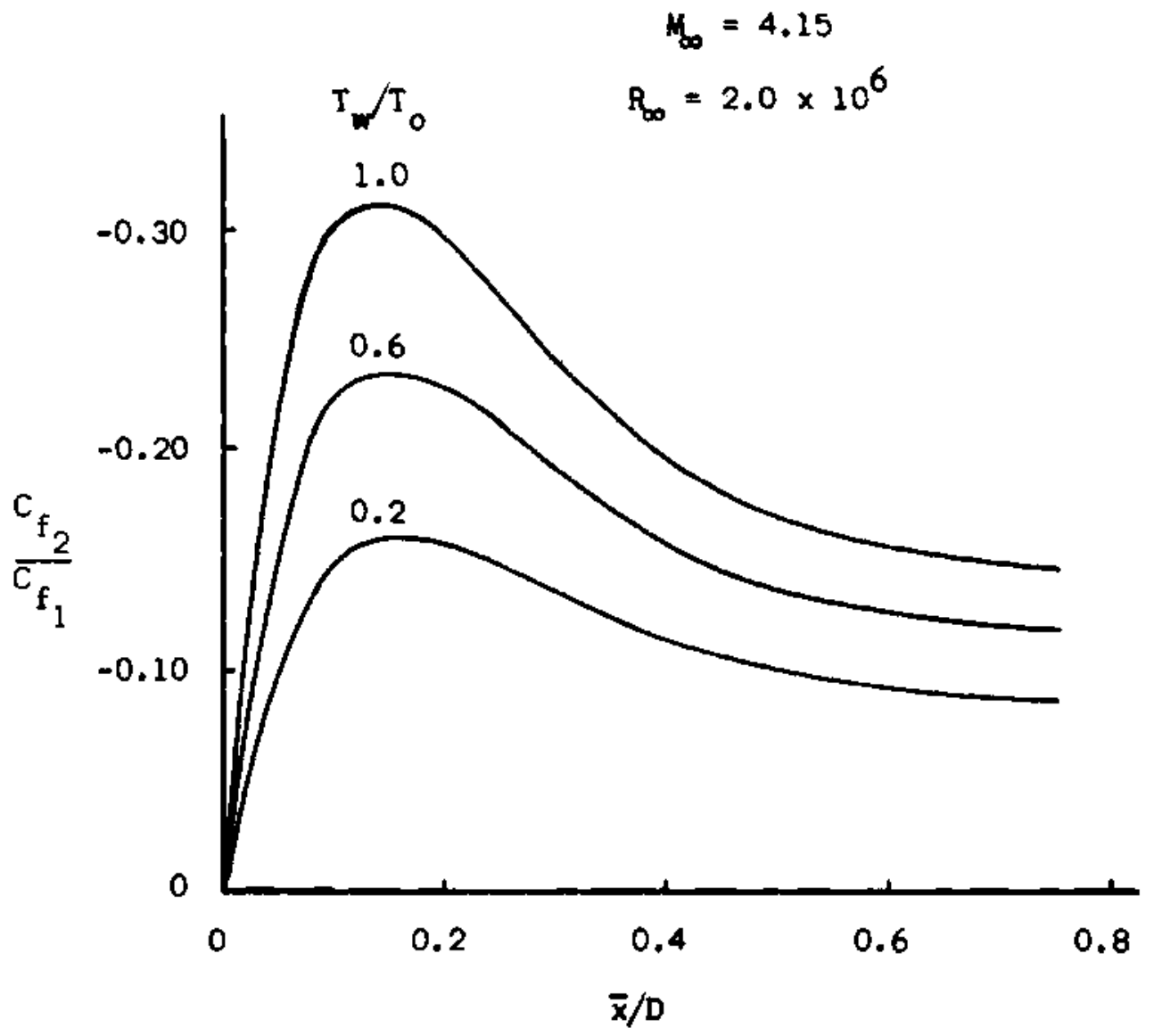

Figure 23. Effect of Cooling on the Cross-Fiow Solution, $\Lambda=10^{\circ}$. 


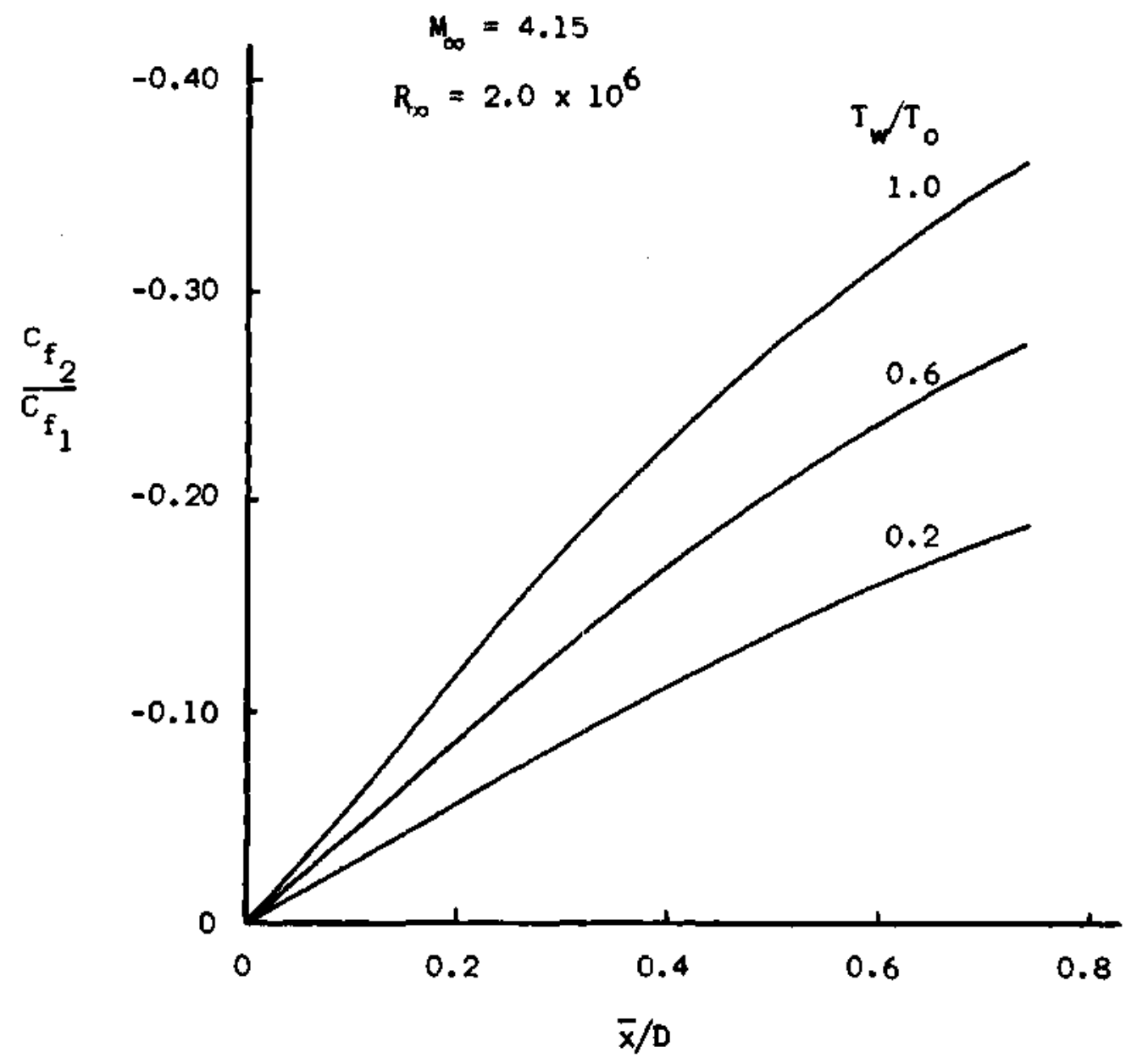

Figure 24. Effect of Cooling on the Cross-Flow Solution, $\Lambda=60^{\circ}$. 


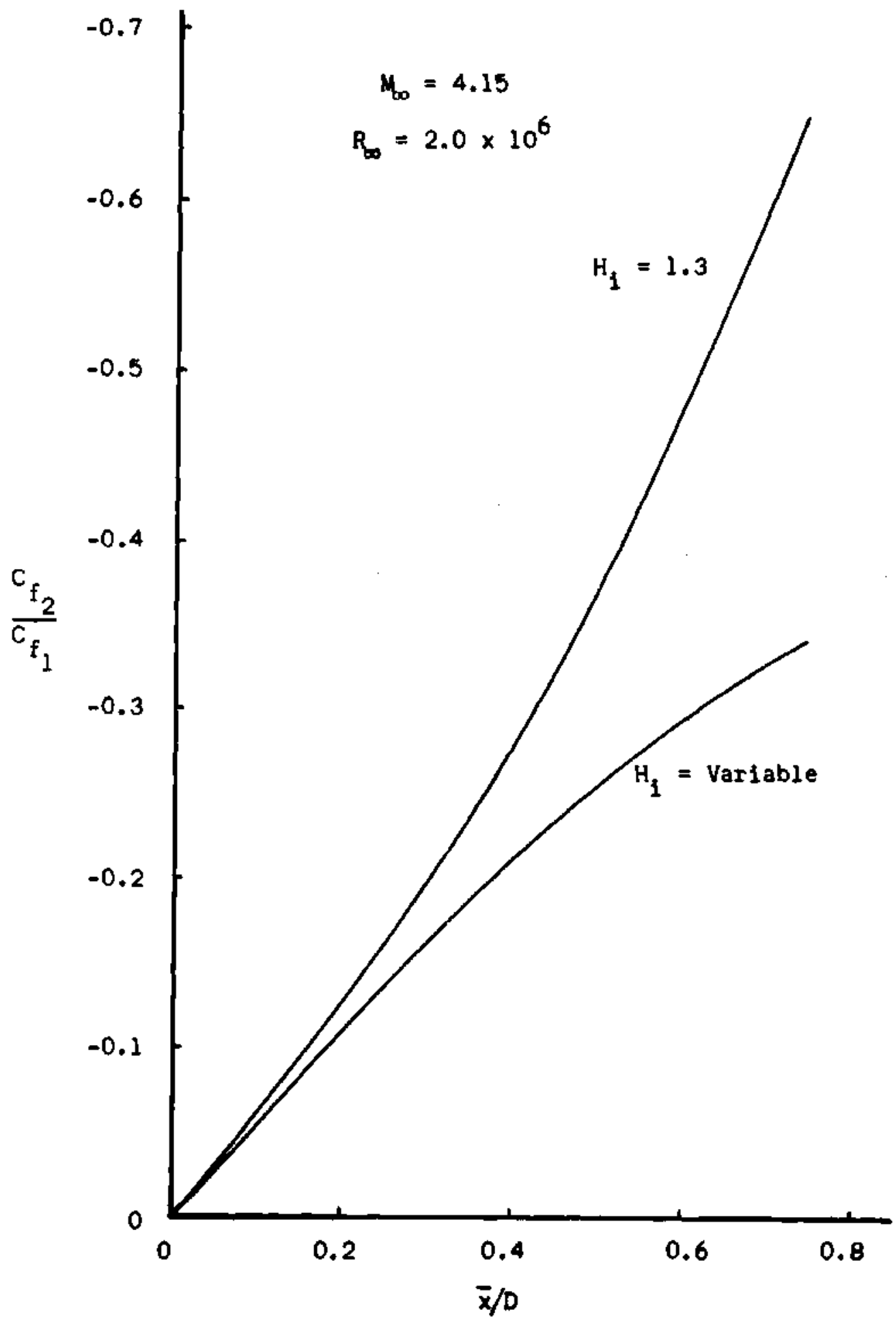

Figure 25. Comparison with Solution Holding $H_{1}$ 
when $H_{i}$ is held constant. This result is to be expected physically since holding $\mathrm{H}_{i}$ constant results in an assumed streamwise velocity profile which does not fill near the wall, as it should under the favor able pressure gradient of this example. Then the cross-flow in this instance does not "feel" the retarding effect of the developing streamwise flow.

The qualitative comparison in this figure indicates that the cross-flow solution is dependent to a significant degree on the form factor parameter $H_{i}$. The statement made previously concerning the importance of employing a streamwise computation method which accounts for the pressure gradient effect on the streamise velocity profile when cross-flow solutions are desired is substantiated by this result. 


\section{CHAPTER $V$}

\section{CONCLUSIONS AND RECOMMENDAT IONS}

The three-dimensional, turbulent boundary layer in compressible flow has been studied analytically from the standpoint of practical computation of engineering parameters assuming small secondary flow within the boundary layer. Procedures for solving the governing integral equations for both the streamwise flow and the cross-flow under general inviscid flow and heat transfer conditions have been proposed. The proposed calculation methods have been applied to a yawed infinite cylinder exam ple where comparisons have been made with experimental heat transfer data, and the effects of several parameters on the cross-flow solution have been considered. The following conclusions are drawn:

1. The assumption of small secondary flow in a streamline coordinate system reduces the equations of continuity, momentum and energy for flow over an arbitrary three-dimensional surface to those analogous to flow over an axially symmetric body where the radius of the body is given by a metric coefficient of the streamline coordinate system. Thus the integral equations along a particular inviscid streamline may be solved, employing experience gained in treatment of twodimensional boundary layers, independent of the cross-flow momentum equation.

2. The application of a recent method given by Sasman and Cresci (33) permits calculation of the streamwise flow over adiabatic surfaces or 
isothermal surfaces with heat transfer in the presence of streamuise pressure gradient. Improvement over previous methods results from a more complete description of pressure gradient effects through the moment-of-momentum equation, which remains coupled with the momentum equation in the Sasman and Cresci method.

3. The cross-flow momentum equation may be simplified by the Magertype transformation employed in the treatment of the streamwise flow, thus permitting straightforward solution of this equation subject to the streamwise solution.

4. Solutions of the cross-flow momentum integral equation provide a test of the validity of the small cross-flow assumption.

5. Application of the proposed computation procedure to a yawed infinite cylinder example in a Mach number 4.15 flow resulted in the following observations:

(a) Nusselt number computed from the solutions along the stagnation streamline assuming a flat plate Reynolds analogy agreed well with experimental data for yaw angles from 10 to 60 degrees and free stream Reynolds numbers from $1 \times 10^{6}$ to $3.5 \times 10^{6}$.

(b) Over the forward face of the cylinder the computed Nusselt number distribution agreed well with the experimental data for yaw angles of 40 and 60 degrees, but agreement was poor for the lower yaw angles of 10 and 20 degrees. The use of the flat plate Reynolds analogy for the low yaw cases, where the streamwise pressure gradient is very much greater than for the 40 and 60 degree yaw angles, is considered 
questionable.

(c) Solutions to the cross-flow momentum equation indicated that the secondary flow was not large for the four yaw angles considered. As a result, the cross-flow effect was expected to be small. This was confirmed by the good agreement of the streamwise solution with heat transfer data at the high $y$ aw angles, where the cross-flow was the greatest.

(d) The cross-flow solution was found to be affected significantly by the assumed form of the cross-flow velocity profile, thus indicating a need for experimental data to serve as basis for assumed profile relations under arbitrary flow conditions.

(e) Increased wall cooling was found to reduce the computed magnitude of the cross-flow, as would be expected from physical considerations.

(f) The cross-flow solution was found to be closely coupled with the development of the streamwise velocity profile as measured by $\mathrm{H}_{i}$, thus indicating the importance of employing a streamwise solution method which accounts for pressure gradient effect on the streamwise velocity profile when cross-flow solutions are considered.

The approximate treatment presented in this work relies on the assumption of small cross-flow as well as on other assumptions based on experimental evidence. This fact coupled with the lack of detailed measurements in compressible three-dimensional flow leads to the obvious 
recommendation that experimental work be undertaken in this area. In particular, measurements of velocity and temperature profiles within the boundary layer are needed to confirm velocity profile assumptions, particularly cross-flow velocity, and to substantiate relationships between velocity and temperature. Independent measurements of skin friction and heat transfer for the same surface geometry and flow conditions are desired to investigate Reynolds analogy for two-dimensional flows with strong pressure gradient as well as general three-dimensional flows.

Iwo observations made during the course of the work seem to be worthy of mention in the form of possibilities rather than recommendations. The first of these concerns two-dimensional flows or equivalently streamise flow with small secondary flow. Since both the Crocco velocity temperature relation and the (related) Reynolds analogy are in some doubt for flows with heat transfer and pressure gradient, the possibility occurs of adding a single parameter to the velocity-temperature relation (e.g. a cubic relation with an undetermined coefficient) then solving the energy integral equation along with the momentum and moment-of-momentum equations. The result would reduce to three coupled differential equations from which a Reynolds analogy parameter dependent on pressure gradient and the degree of cooling would evolve. This type of approach has been tried by Cohen (31), for example, with some success although in this case the form factor was assumed constant. Of course, the form of the assumed velocity-temperature relation should be based on experiment (as presented in summary form by Rotta (38), for example). The advantage of this approach rests in the fact that the solutions thus obtained satisfy the energy integral equation as well as the momentun equation. 
A second observation stems from the experimental data of Hall (24) and concerns the form of the cross-flow velocity profile. In previous discussion it was noted that, based on Hall's data, the parabolic relation gave a reasonable approximation for favorable streamwise pressure gradient but was inadequate when the pressure gradient became adverse. Hall noted that the turbulent boundary layer appeared to differ from the laminar case in that the cross-over (or s-shaped) cross-flow profile did not occur when the streamwise pressure gradient changed from favorable to adverse along a streamline. Further, these data indicate that a cubic or higher power profile relation of the form used in the present work may provide a reasonable approximation in the adverse gradient region of the flow. These facts suggest the possibility of defining a single-parameter family of cross-flow velocity profiles dependent on streamwise pressure gradient much the same as the $\mathrm{H}_{i}$ parametric representation of the streamwise flow. This suggestion must be considered highly tentative and requires far more experimental verification than is presently available. 
APPENDIX A

BOUNDARY LAYER INTEGRAL EQUATIONS

The integral equations for three-dimensional, compressible, turbulent boundary layers are presented in this appendix, both for the general case in streamline coordinates and for the small cross-flow case. The thickness functions are defined and the key steps in the derivation are outlined.

The following momentum and displacement thickness functions are defined in a streamline coordinate system.

$$
\begin{array}{ll}
\theta_{11}=\int_{0}^{\delta}\left(1-\frac{u}{u_{e}}\right) \frac{\rho u}{\rho_{e} u_{e}} d \zeta & \theta_{12}=\int_{0}^{\delta}\left(1-\frac{u}{u_{e}}\right) \frac{\rho v}{\rho_{e} u_{e}} d \zeta \\
\theta_{21}=-\int_{0}^{\delta} \frac{\rho u v}{\rho_{e} u_{e}^{2}} d \zeta & \theta_{22}=-\int_{0}^{\delta} \frac{\rho v^{2}}{\rho_{e} u_{e}^{2}} d \zeta \\
\delta_{1}^{*}=\int_{0}^{\delta}\left(1-\frac{\rho u}{\rho_{e} u_{e}}\right) d \zeta & \delta_{2}^{*}=-\int_{0}^{\delta} \frac{\rho v}{p_{e} u_{e}} \alpha
\end{array}
$$

where $\delta$ is a hypothetical boundary layer thickness taken as greater than the velocity and enthalpy thicknesses of the boundary layer. of course the integration limits could as well be from zero to infinity without changing the results. The enthalpy convection thicknesses are defined as 


$$
\begin{aligned}
& \varphi_{1}=\int_{0}^{\delta}\left(1-\frac{\mathrm{H}_{i}^{*}}{\mathrm{H}_{e}}\right) \frac{\rho \mathrm{u}}{\rho_{e}^{\mathrm{u}_{e}}} d \zeta \\
& \varphi_{2}=\int_{0}^{\delta}\left(1-\frac{\mathrm{H}^{*}}{\mathrm{H}_{e}}\right) \frac{\rho \mathrm{v}}{\rho_{e} \mathrm{u}_{e}} d \zeta
\end{aligned}
$$

where $\mathrm{H}^{*}=\mathrm{H}-\mathrm{H}_{\mathrm{w}}$.

The integral equations are derived in the usual way, by integrating equations 3, 4, and 6 term by term across the boundary layer. The momentum integral equations have been given by Cooke and Hall (3) for general three-dimensional flow and the energy integral equation is added here for completeness. The steps in the derivation of these equations are outlined.

The terms involving $p$ are eliminated using equations 12 and 13. Then integration of the continuity equation (equation 2) is performed to eliminate the term $\left[w+\left(\overline{\rho^{\prime} w^{\prime}}\right) / \rho\right]$ from the remaining equations. Thus

$$
w+\frac{\left(\overline{\rho^{\prime} w^{t}}\right)}{\rho}=\frac{-1}{\rho e_{1} e_{2}} \int_{0}^{\zeta}\left[\frac{\partial}{\partial \xi}\left(\rho_{1} e^{u}\right)+\frac{\partial}{\partial \eta}\left(\rho e_{1} v\right)\right] d \zeta
$$

is substituted into equations 3,4 , and 6 .

One notes that

$$
\int_{0}^{\delta} \frac{\partial}{\partial \zeta}\left[\mu \frac{\partial u}{\partial \zeta}-\rho\left(\overline{u^{\prime} w^{\top}}\right)\right] d \zeta=-\tau_{1}
$$

and

$$
\int_{0}^{\delta} \frac{\partial}{\partial \zeta}\left[\mu \frac{\partial v}{\partial \zeta}-\rho\left(\overline{v^{\prime} w^{\prime}}\right)\right] d \zeta=-\tau_{2}
$$


where $\tau_{1_{w}}$ and $\tau_{2_{w}}$ are the local components of shear stress at the wall in the $\xi$ and $\eta$ directions, respectively. Also

$$
\int_{0}^{\delta} \frac{\partial}{\partial \zeta}\left[\mu\left(\frac{\partial H}{\partial \zeta}+\frac{1-P I}{P r} \frac{\partial h}{\partial \zeta}\right)-p\left(\overline{w^{\prime} H^{\prime}}\right)\right]=q_{w}
$$

where $q_{w}$ is the heat transferred to the surface. Here the perfect gas assumption is employed, $h=h(T)=c_{p} T$.

Now integration of equations 3,4 , and 6 across the boundary layer (from zero to 8 ) and application of the relations given by equations A.1 through A. 6 yields the boundary layer integral equations. The details are tedious, but straightforward. The resulting equations applicable for three-dimensional boundary layers in streamline coordinates are $\xi$-Momentum:

$$
\begin{aligned}
& \frac{1}{e_{1}} \frac{\partial \theta_{11}}{\partial \xi}+\left[\frac{1}{e_{1} \rho_{e}} \frac{\partial \rho_{e}}{\partial \xi}+\frac{1}{e_{1} u_{e}} \frac{\partial u_{e}}{\partial \xi}\left(2+\frac{\delta_{1}^{*}}{\theta_{11}}\right)-k_{1}\right] \theta_{11} \\
& +\frac{1}{e_{1}} \frac{\partial \theta_{12}}{\partial \eta}+\left[\frac{1}{e_{2} p_{e}} \frac{\partial \rho_{e}}{\partial \eta}+\frac{1}{e_{2} u_{e}} \frac{\partial u_{e}}{\partial \eta} 2+\frac{\delta}{\theta_{12}^{*}}-k_{2}\right] \theta_{12} \\
& +k_{1} \theta_{22}-k_{2} \theta_{21}=\frac{\tau_{1}}{\rho_{e} u_{e}}
\end{aligned}
$$

$\eta$-Momentum:

$$
\begin{aligned}
& \frac{1}{e_{1}} \frac{\partial \theta_{21}}{\partial \xi}+\left[\frac{1}{e_{1} \rho_{e}} \frac{\partial \rho_{e}}{\partial \xi}+\frac{2}{u_{e}} \frac{\partial u_{e}}{\partial \xi}-2 K_{1}\right] \theta_{21}+k_{2}\left(\theta_{11}+s_{1}^{*}\right) \\
& +\frac{1}{e_{2}} \frac{\partial \theta_{22}}{\theta_{\eta}}+\left[\frac{1}{e_{2} \rho_{e}} \frac{\partial \rho_{e}}{\partial \eta}+\frac{2}{u_{e}} \frac{\partial u_{e}}{\partial \eta}-k_{2}\right] \theta_{22}=\frac{{ }_{2}{ }_{w}}{\rho_{e} u_{e}{ }^{2}}
\end{aligned}
$$


Energy:

$$
\begin{aligned}
& \frac{1}{e_{1}} \frac{\partial \varphi_{1}}{\partial E}+\left[\frac{1}{e_{1} H_{e}^{*}} \frac{\partial H_{e}^{*}}{\partial t}+\frac{1}{e_{1} u_{e}} \frac{\partial u_{e}}{\partial \tau}+\frac{1}{e_{1} \rho_{e}} \frac{\partial \rho_{e}}{\partial t}-K_{1}\right] \omega_{1}+\frac{1}{e_{2}} \frac{\partial \varphi_{2}}{\partial \eta} \quad \text { (A.9) } \\
& +\left[\frac{1}{e_{2} H_{e}^{*}} \frac{\partial H_{e}^{*}}{\partial \eta}+\frac{1}{e_{2} u_{e}} \frac{\partial u_{e}}{\partial \eta}+\frac{1}{e_{2} \rho_{e}} \frac{\partial \rho_{e}}{\partial \eta}-K_{2}\right] \varphi_{2}=\frac{-q_{w}}{\rho_{e} u_{e} H_{e}^{*}}
\end{aligned}
$$

The small cross-flow equations are derived in exactly the same manner from the simplified partial differential equations. These are E-Momentum:

$$
\frac{1}{e_{1}} \frac{\partial \theta_{11}}{\partial \xi}+\left[\left(2+\frac{\delta_{1}^{*}}{\theta_{11}}\right) \frac{1}{e_{1} u_{e}} \frac{\partial u_{e}}{\partial E}+\frac{1}{e_{1} \rho_{e}} \frac{\partial \rho_{e}}{\partial \xi}-k_{1}\right] \theta_{11}=\frac{\tau_{1}}{\rho_{e} u_{e}^{2}} \text { (A.10) }
$$

$\eta$-Momentum:

$$
\begin{gathered}
\frac{1}{e_{1}} \frac{\partial \theta_{2 l}}{\partial t}+\left[\frac{2}{e_{1} u_{e}} \frac{\partial u_{e}}{\partial t}+\frac{1}{e_{1} p_{e}} \frac{\partial p_{e}}{\partial \xi}-2 k_{1}\right] \theta_{21} \\
+k_{2}\left(\theta_{11}+s_{1}^{*}\right)=\frac{\tau_{2} w_{e}}{p_{e} u^{2}}
\end{gathered}
$$

Energy:

$$
\frac{1}{e_{1}} \frac{\partial \varphi_{i}}{\partial \xi}+\left[\frac{1}{e_{1} H_{e}^{*}} \frac{\partial H_{e}^{*}}{\partial \xi}+\frac{1}{e_{1} u_{e}} \frac{\partial u_{e}}{\partial \xi}+\frac{1}{e_{1} \rho_{e}} \frac{\partial \rho_{e}}{\partial \xi}-K_{1}\right] \varphi_{1}=\frac{-q_{w}}{\rho_{e} u_{e} H_{e}^{*}}
$$


APPENDIX B

TRANSFORMATION OF THE CROSS-FLOW MOMENTUM EQUAT ION

The $\eta$-momentum equation may be written as

$$
\begin{gathered}
\rho u \frac{\partial v}{\partial s}+\left[\rho w+\left(\overline{\rho^{\prime} w^{\top}}\right)\right] \frac{\partial v}{\partial \zeta}-\frac{\rho u v}{r} \frac{d r}{d s} \\
-k_{2}\left(\rho_{e} u_{e}^{2}-\rho u^{2}\right)=\frac{\partial \tau_{2}}{\partial \zeta}
\end{gathered}
$$

and the continuity equation as

$$
\frac{\partial}{\partial s}(\rho u r)+\frac{\partial}{\partial \zeta}\left[\rho w r+\left(\overline{\rho^{\prime} w^{\prime}}\right) r\right]=0
$$

where $\tau_{2}$ is the virtual turbulent shear stress given by

$$
\tau_{2}=\mu \frac{\partial u}{\partial \zeta}+\rho\left(\overline{u^{\top} w^{\top}}\right)
$$

and small cross-flow has been assumed.

The Mager-type transformation of Sasman and Cresci (33) is given by equations 23 and 24 in Chapter III, that is

$$
s=\int_{0}^{s} a(s) d s
$$

and

$$
z=b(s) \int_{0}^{\zeta} \frac{\rho}{\rho_{0}} d \zeta
$$


It follows then that

$$
\frac{\partial}{\partial s}=a \frac{\partial}{\partial S}+\frac{\partial Z}{\partial s} \frac{\partial}{\partial Z}
$$

and

$$
\frac{\partial}{\partial \zeta}=b \frac{\rho}{\rho_{c}} \frac{\partial}{\partial Z}
$$

Following Mager (25) define a stream function which is invariant under transformation so that $\psi=\bar{\phi}$. Then

$$
\text { rpu }=\rho_{0} \frac{\partial \psi}{\partial \zeta} \quad I \rho w^{\prime}+r\left(\overline{\rho^{\prime} w^{\top}}\right)=-\rho_{0} \frac{\partial \psi}{\partial s}
$$

and

$$
r U=\frac{\partial \bar{\psi}}{\partial Z} \quad r w=-\frac{\partial \bar{\psi}}{\partial S}
$$

where $U, W$, and $\bar{\psi}$ are the transformed velocities and stream function.

Application of the transformation gives

$$
\mathrm{u}=\mathrm{bU}
$$

and

$$
r\left[\rho w+\left(\overline{\rho^{\top} w^{T}}\right)\right]=\rho_{0} a r w-\rho_{0} r U \frac{\partial Z}{\partial s}
$$

Define for small cross-flow

$$
\mathrm{v}=\mathrm{v}
$$


That is, the cross-flow velocity in the transformed plane is equal to the compressible velocity.

Applying the transform relations B.5 through B. 9 to equation B.1 and simplifying gives

$$
U \frac{\partial V}{\partial S}+W \frac{\partial V}{\partial Z}+\frac{U V}{r} \frac{\partial \mathbf{r}}{\partial S}-\frac{k_{2} b}{a}\left[\frac{\rho}{\rho} U_{e}^{2}-U^{2}\right]=\frac{1}{\rho_{0} a} \frac{\partial \tau_{2}}{\partial Z}
$$

Assuming a perfect gas, $\frac{\rho_{e}}{\rho}=\frac{h}{h_{e}}$, and writing

$$
\bar{K}_{2}=\frac{k_{2}}{a b}
$$

equation $\mathrm{B}, 10$ becomes after some manipulation

$$
U \frac{\partial V}{\partial S}+W \frac{\partial V}{\partial Z}+\frac{U V}{r} \frac{\partial r}{\partial S}-\bar{K}_{2}\left(\frac{H}{H_{e}} U_{e}^{2}-U^{2}\right)=\frac{1}{\partial p_{o}} \frac{\partial \tau_{2}}{\partial Z}
$$

The above is an incompressible plane momentum equation where one notes that for adiabatic flow $\mathrm{H} / \mathrm{H}_{\mathrm{e}}=1$.

The transformed momentum integiral equation is derived in the usual manner, making use of the transformed continuity equation

$$
\frac{\partial U}{\partial S}+\frac{\partial W}{\partial Z}+\frac{U}{r} \frac{\partial r}{\partial S}=0
$$

and integrating equation $\mathrm{B} .12$ term by term from $\mathrm{Z}=0$ to $\mathrm{Z}=\Delta$. The details are straightforward and are omitted here. The resulting equation is

$$
\frac{d \bar{\theta}_{21}}{d S}+\bar{\theta}_{21}\left[\frac{2}{U_{e}} \frac{d U_{e}}{d S}+\frac{2}{r} \frac{d r}{d S}\right]+\bar{K}_{2}\left(\bar{\theta}_{11}-\Delta_{l}^{*}\right)=\frac{1}{a} \frac{\tau_{2}}{\rho_{o} U_{e}^{2}}
$$


where

$$
\bar{\theta}_{21}=-\int_{0}^{\Delta} \frac{\mathrm{uV}}{\mathrm{U}_{\mathrm{e}}^{2}} \mathrm{dz}
$$

and where $\bar{\theta}_{11}$ and $\Delta_{1}^{*}$ are defined in the text by equations 30 and 31 . It is noted that equation B.14 can also be derived by transforming the compressible $\eta$-momentum integral equation given by equation 21 . 


\section{APPENDIX C \\ THE CROSS-FLOW VELOCITY PROFILE AND RESULTING \\ MOMENTUM INTEGRAL EQUATION}

The cross-flow momentum integral equation is reduced to a form for solution which is consistent with the streamwise solution method. The parabolic profile relation is assumed as given by equation 50 in the text. That is

$$
\frac{\mathrm{V}}{\mathrm{U}_{\mathrm{e}}}=\left(1-\frac{\mathrm{Z}}{\Delta}\right)^{2} \frac{\mathrm{U}}{\mathrm{U}_{\mathrm{e}}} \mathrm{A}
$$

where

$$
A=\tan \bar{a}
$$

and is a function of $s$. The streamwise velocity profile is given by equation 29 as

$$
\frac{\mathrm{U}}{\mathrm{U}_{\mathrm{e}}}=\left(\frac{\mathrm{Z}}{\Delta}\right)^{\mathrm{N}_{\mathrm{i}}}
$$

Consider now the transformed momentum integral equation in the form of equation 49.

$$
\begin{gathered}
\frac{d \bar{\theta}_{21}}{d S}+\bar{\theta}_{21}\left[\frac{2}{U_{e}} \frac{d U_{e}}{d S}+\frac{2}{r} \frac{d r}{d S}\right]+\bar{K}_{2} \bar{\theta}_{11}\left(1+\frac{T_{w}}{T_{0}} H_{i}\right) \\
=\frac{T_{r}}{I_{0}} \frac{C_{f_{l}}}{2} A
\end{gathered}
$$


The definition of $\bar{\theta}_{11}$ is employed along with equation C.3 to give

$$
\frac{\bar{\theta}}{\Delta}=\int_{0}^{1} \frac{U}{U_{e}}\left(1-\frac{U}{U_{e}}\right) d\left(\frac{Z}{\Delta}\right)=\frac{N_{i}}{\left(N_{i}+1\right)\left(2 N_{i}+1\right)}
$$

or since $\mathrm{H}_{i}=2 \mathrm{~N}_{i}+1$ (equation 33 )

$$
\frac{\bar{\theta}}{\Delta}=\frac{\left(H_{1}-1\right)}{H_{i}\left(H_{i}+1\right)}
$$

Now the definition of $\bar{\theta}_{21}$

$$
\frac{\bar{\theta}_{21}}{\Delta}=-\int_{0}^{1} \frac{\mathrm{vu}}{\mathrm{u}_{\mathrm{e}}^{2}} d\left(\frac{\mathrm{z}}{\Delta}\right)
$$

along with equations $C .1$ and $C .3$ yields after integration

$$
\frac{\bar{\theta}_{21}}{\Delta}=\frac{-2 A}{H_{i}\left(H_{1}+1\right)\left(H_{i}+2\right)}
$$

Then $\bar{\theta}_{21}$ and $\bar{\theta}_{11}$ are related by

$$
\bar{\theta}_{21}=\frac{-2 A \bar{\theta}_{11}}{\left(H_{i}-1\right)\left(H_{i}+2\right)}
$$

and

$$
\frac{d \bar{\theta}_{21}}{d S}=\frac{-2 A \bar{\theta}_{11}}{\left(H_{i}-1\right)\left(H_{i}+2\right)}\left[\frac{1}{A} \frac{d A}{d S}+\frac{1}{\bar{\theta}_{11}} \frac{d \bar{\theta}_{11}}{d S}-\frac{2 H_{i}+1}{\left(H_{i}-1\right)\left(H_{i}+2\right)} \frac{d H_{i}}{d S}\right]
$$

Substitution of equations $C .9$ and $C .10$ into $C .4$ gives 


$$
\begin{aligned}
\frac{d A}{d S}= & -A\left[\frac{1}{\bar{\theta}_{11}} \frac{d \bar{\theta}_{11}}{d S}+\frac{2}{U_{e}} \frac{d U_{e}}{d S}+\frac{2}{r} \frac{d r}{d S}-\frac{\left(2 H_{i}+1\right)}{\left(H_{i}-1\right)\left(H_{i}+2\right)} \frac{d H_{i}}{d S}\right. \\
& \left.+\frac{\left(H_{i}-1\right)\left(H_{1}+2\right)}{2 \bar{\theta}_{11}} \frac{T_{r}}{T_{0}} \frac{C_{f}}{2}\right]+\bar{K}_{2} \frac{\left(H_{i}-1\right)\left(H_{i}+2\right)}{2}\left(1+\frac{T_{w}}{T_{0}} H_{i}\right)
\end{aligned}
$$

Writing the equation in terms of the physical distance along the surface, $s$, and introducing the relations for $\mathrm{C}_{\mathrm{f}_{1}}, \overline{\mathrm{K}}_{2}$, and $\mathrm{f}$ as given by equations 27,45 , and 38 in the text, the momentum integral equation becomes

$$
\begin{aligned}
\frac{d A}{d s}= & -A\left[\frac{1}{1.268 f} \frac{d f}{d s}+\frac{1}{M_{e}} \frac{d M_{e}}{d s}+\frac{2}{r} \frac{d r}{d s}-\frac{\left(2 H_{i}+1\right)}{\left(H_{i}-1\right)\left(H_{i}+2\right)} \frac{d H_{i}}{d s}\right. \\
& \left.+\frac{\left(H_{i}-1\right)\left(H_{i}+2\right)}{2 f} B\right]+k_{2}\left(\frac{T_{0}}{T_{e}}\right)^{\frac{1}{2}} \frac{\left(H_{i}-1\right)\left(H_{i}+2\right)}{2}\left(1+\frac{T_{w}}{I_{0}} H_{i}\right)
\end{aligned}
$$

where

$$
B=0.123 e^{-1.561 H_{i}} \quad \frac{\mu_{e} a_{0}}{v_{0}} \quad \frac{T_{e}}{T_{r}}\left(\frac{T_{e}}{T_{0}}\right)^{3}\left(\frac{\mu_{r}}{\mu_{0}}\right)^{0.268}
$$

Equation $C .12$ is now formulated in the same notation as that of the streamwise equations, equation 39 and 40 .

In view of the uncertainty connected with the cross-flow velocity profile approximation, as discussed in Chapter III, two alternate profiles of the same general form as equation $C . I$ are considered in the numerical example of Chapter IV. The corresponding momentum integral equations are developed here. 
Consider a "linear" and a "cubic" velocity profile relation to be given by

$$
\frac{V}{U_{e}}=\left(1-\frac{Z}{\Delta}\right) \frac{U}{U_{e}} A
$$

and

$$
\frac{V}{U_{e}}=\left(1-\frac{Z}{\Delta}\right)^{3} \frac{U}{U_{e}} A
$$

respectively. The resulting relations between $\bar{\theta}_{21}$ and $\bar{\theta}_{11}$ become for the linear relation,

$$
\bar{\theta}_{21}=\frac{-\mathrm{A} \bar{\theta}_{11}}{H_{1}-1}
$$

and for the cubic relation,

$$
\bar{\theta}_{21}=\frac{6 A \bar{\theta}_{11}}{\left(H_{i}-1\right)\left(H_{i}+2\right)\left(H_{i}+3\right)}
$$

Using these relations the resulting momentum integral equations are derived in the same manner as was done for the parabolic assumption. The final equations may be written as

$$
\begin{aligned}
\frac{d A}{d s}=-A & {\left[\frac{1}{1.268 f} \frac{d f}{d s}+\frac{1}{M_{e}} \frac{d M_{e}}{d s}+\frac{2}{r} \frac{d r}{d s}=F \frac{d H_{i}}{d s}\right.} \\
& \left.+G \frac{B}{f}\right]+G K_{2}\left(\frac{T_{o}}{I_{e}}\right)^{\frac{1}{2}}\left(1+\frac{T_{w}}{T_{o}} H_{i}\right)
\end{aligned}
$$


where $B$ is given by equation $C .13$ and $F$ and $G$ are dependent on the profile assumption as follows:

$\underline{F}$

Linear Relation

Parabolic Relation

Cubic Relation

$$
\frac{1}{\left(H_{1}-1\right)}
$$

$$
\frac{\left(2 \mathrm{H}_{i}+1\right)}{\left(\mathrm{H}_{i}-1\right)\left(\mathrm{H}_{i}+2\right)}
$$

$$
\frac{\left(3 \mathrm{H}_{j}^{2}+8 \mathrm{H}_{i}+1\right)}{\left(\mathrm{H}_{i}-1\right)\left(\mathrm{H}_{i}+2\right)\left(\mathrm{H}_{i}+3\right)}
$$

$\underline{G}$

$$
\left(H_{1}-1\right)
$$

$$
\frac{\left(H_{i}-1\right)\left(H_{1}+2\right)}{2}
$$

$\frac{\left(H_{1}-1\right)\left(H_{i}+2\right)\left(H_{i}+3\right)}{6}$ 
APPENDIX D

\section{GEOMETRIC PROPERTIES FOR A YAWED INF INITE CYLINDER}

Consider a yawed infinite cylinder with the coordinate systems $\bar{x}, \bar{y}$ and $s, \eta$ defined on the surface as shown in Figure 2. Here $s$ is the trace of a particular inviscid streamline on the cylinder surface and $\eta$ is everywhere orthogonal to s. The $\bar{x}, \bar{y}$ coordinates are as defined in the text, $\bar{y}$ lies along the cylinder generators and $\bar{x}$ is normal to this direction. Elements of length dL on the surface are given by

$$
\mathrm{dL}^{2}=d \overline{\mathrm{x}}^{2}+\mathrm{d} \overline{\mathrm{y}}^{2}
$$

for the $\bar{x}, \bar{y}$ system and

$$
d L^{2}=d s^{2}+I^{2} d \eta^{2}
$$

for the $s, \eta$ system. The latter is consistent with the metric relation of the streamline coordinate system described by equations 1 in the text for points lying in the surface $(\zeta=0)$ and where it is recalled that $r$ is defined as equal to $e_{2}$

The velocity components in the two systems are determined from the Mach number distribution $M_{e}(\bar{x})$ as given by equations 66 and 68 in Chapter IV. In the $\bar{x}, \bar{y}$ system note that $\bar{v}_{e}=$ constant for the infinite cylinder, then 


$$
\frac{\bar{v}_{e}}{a_{0}}=M_{\infty}\left(\frac{T_{\infty}}{T_{0}}\right)^{\frac{1}{2}} \sin \Lambda=M_{s l}\left(\frac{T_{s l}}{T_{0}}\right)^{\frac{1}{2}}
$$

and

$$
\frac{\bar{u}_{e}}{a_{0}}=\left[m_{e}^{2} \frac{T_{e}}{I_{0}}-\left(\frac{\bar{v}_{e}}{a_{0}}\right)^{2}\right]^{\frac{l}{2}}
$$

where $a_{0}=\sqrt{r \mathrm{RT}_{0}}$. In the $s, \eta$ system

$$
v_{e}=0
$$

and

$$
\frac{u_{e}}{a_{o}}=M_{e}\left(\frac{T_{e}}{T_{0}}\right)^{\frac{1}{2}}
$$

The equation of the streamline on the surface is

$$
\frac{d \bar{y}}{d \bar{x}}=\frac{\bar{v}_{e}}{\bar{u}_{e}}
$$

It is evident from the flow geometry that the physical length elements and velocities are related by

$$
\frac{d s}{d \bar{x}}=\frac{u_{e}}{\bar{u}_{e}}
$$

and

$$
\frac{d n}{d \bar{x}}=-\frac{u_{e}}{\bar{v}_{e}}
$$


Since $\frac{\partial}{\partial \bar{y}}=0$ the following relations follow

$$
\frac{\partial}{\partial s}=\frac{\bar{u}_{e}}{u_{e}} \frac{\partial}{\partial \bar{x}}
$$

and

$$
\frac{\partial}{\partial n}=-\frac{\bar{v}_{e}}{u_{e}} \frac{\partial}{\partial \bar{x}}
$$

The distance $s(\bar{x})$ may be determined by integrating equation $D .8$ and the corresponding coordinate $\bar{y}$ from an integration of D.7.

Calculation of the streamline divergence term $\frac{l}{r} \frac{\mathrm{dr}}{\mathrm{ds}}$ is now possible considering the invariant nature of the divergence of the velocity vector, which is everywhere tangent to the surface. In indicial notation the divergence of a velocity vector $\vec{v}$ with components $v^{i}$ is

$$
\operatorname{div} \vec{V}=\frac{1}{\sqrt{g}} \frac{\partial}{\partial x^{i}}\left(\sqrt{g} v^{i}\right)
$$

as given by Spain (41) for example. Here $g$ is the determinate of the fundamental tensor of the surface metric. Thus in the $\bar{x}, \bar{y}$ coordinate system with the metric given by equation D.l one finds $g=1$ and

$$
\operatorname{div} \vec{v}=\frac{d \bar{u}}{d \bar{x}}
$$

since $\vec{v}_{e}=$ const. In the $s, \eta$ system of equation D.2

$$
g=\left(\begin{array}{ll}
1 & 0 \\
0 & x^{2}
\end{array}\right)=x^{2}
$$


and

$$
\operatorname{div} \vec{v}=\frac{\partial u}{\partial s}+\frac{u}{r} \frac{\partial r}{\partial s}
$$

since $v_{e}=0$. Noting that div $\vec{v}$ is invariant in coordinate transformation one may equate equations D.13 and D.15 to yield

$$
\frac{1}{r} \frac{\partial r}{\partial s}=\frac{1}{u_{e}}\left(\frac{d \bar{u}}{d \bar{x}}-\frac{\partial u_{e}}{\partial s}\right)
$$

On the stagnation line of the cylinder $u_{e}=\bar{v}_{e}=$ constant and

$$
\left|\frac{1}{r} \frac{d r}{d s}\right|_{s l}=\frac{1}{v_{e}}\left(\frac{d \bar{u}_{e}}{d \bar{x}}\right\rangle_{s l}
$$

Since $u_{e}^{2}=\bar{u}_{e}^{2}+\vec{v}_{e}^{2}$, then

$$
u_{e} \frac{\partial u_{e}}{\partial \vec{x}}=\bar{u}_{e} \frac{d \bar{u}_{e}}{d \bar{x}}
$$

Using the above relation and equation D.10 the streamline divergence is written finally as

$$
\frac{1}{r} \frac{d r}{d s}=\frac{\bar{v}_{e}^{2}}{u_{e}^{3}} \frac{d \bar{u}_{e}}{d \bar{x}}
$$

The eurvature parameter $K_{2}$ is determined making use of equation 13 in Chapter II, i.e.

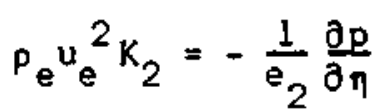


In view of equation D.11 and $e_{2}=r$

$$
\frac{1}{e_{2}} \frac{\partial p}{\partial \eta}=-\frac{\bar{v}_{e}}{u_{e}} \frac{\partial p}{\partial \bar{x}}
$$

Euler's equation in the $\bar{x}, \bar{y}$ system gives

$$
\frac{1}{\rho_{e}} \frac{\partial p}{\partial \bar{x}}=-\vec{u}_{e} \frac{\partial \bar{u}_{e}}{\partial \bar{x}}
$$

Thus

$$
k_{2}=\frac{-\bar{v}_{e} \bar{u}_{e}}{u_{e}^{3}} \frac{d \bar{u}_{e}}{d \bar{x}}
$$

The required geometric parameters for the yawed infinite cylinder are now defined by equations $0.8,0.19$ and 0.23 . 
APPENDIX E

\section{THE REFERENCE TEMPERATURE}

The application of the reference temperature method to obtain a compressible friction formula is based on the postulate that an incompressible formula will correctly compute the compressible skin friction if the properties in this formula are evaluated at some reference temperature, which is characteristic of the compressible flow under consideration. A commonly used relation for computing the reference temperature is one given by Eckert (35).

$$
T_{r}=0.5 \frac{T_{w}}{T_{0}}+0.22 \mathrm{Pr}^{\frac{1}{3}}+\left(0.5-0.22 \mathrm{Pr}^{\frac{1}{3}}\right) \frac{\mathrm{T}_{e}}{T_{0}}
$$

This relation was based upon exact laminar boundary layer solutions for flow over flat plates. Here $\mathrm{Pr}^{1 / 3}$ is the assumed recovery factor for turbulent flow which replaces the laminar recovery factor of the original derivation. The extension to turbulent flow was given by Eckert based on correlations of the Schultz-Grunow incompressible friction law with com-. pressible experimental data for flat plates with adiabatic and heated surfaces.

This relation is often employed for flows with pressure gradient and heat transfer although concrete justification for doing so is lacking. It is noted that the defense of this application a posteriori is often complicated because of numerous other approximations required to obtain analytic solutions such as the Crocco relation, velocity profile assumption, 
and even the assumed form of the incompressible friction law to which the reference temperature method is applied. Further, the scarcity of skin friction data for pressure gradient flows often requires that heat transfer data provide a basis of comparison thus involving a Reynolds analogy a ssumption.

The above simply points out the arbitrary nature of a choice for the reference temperature. A discussion of the various methods found in the literature for estimating the reference temperature is not intended here. Sasman and Cresci (33) use the Eckert relation given by equation E.1. In the present application, that is the yawed infinite cylinder with moderate heat transfer, the proper reference temperature is assumed to be given by the temperature of the wall. This choice is supported by comparing computations using the Sasman and Cresci (33) method with experimental data for an adiabatic flat plate and for two favorable pressure gradient examples, one with adiabatic wall and another with moderate heat transfer to the surface.

In Figure E-l the experimental flat plate skin friction coefficients given by Matting et al. (47) are plotted versus length Reynolds number $R_{x}$ measured from the virtual origin of turbulent flow for free stream Mach numbers of 2.95 and 4.2. These data were measured using a skin friction element for flow of air over an insulated flat plate. The corresponding skin friction coefficient variation computed by the Sasman and Cresci method employing both the Eckert reference temperature and the $T_{r}=T_{w}$ assumption are shown in this figure. The wall temperature in these calculations was taken to be the theoretical recovery temperature which was confirmed as correct by Matting et al. (47). Somewhat better 


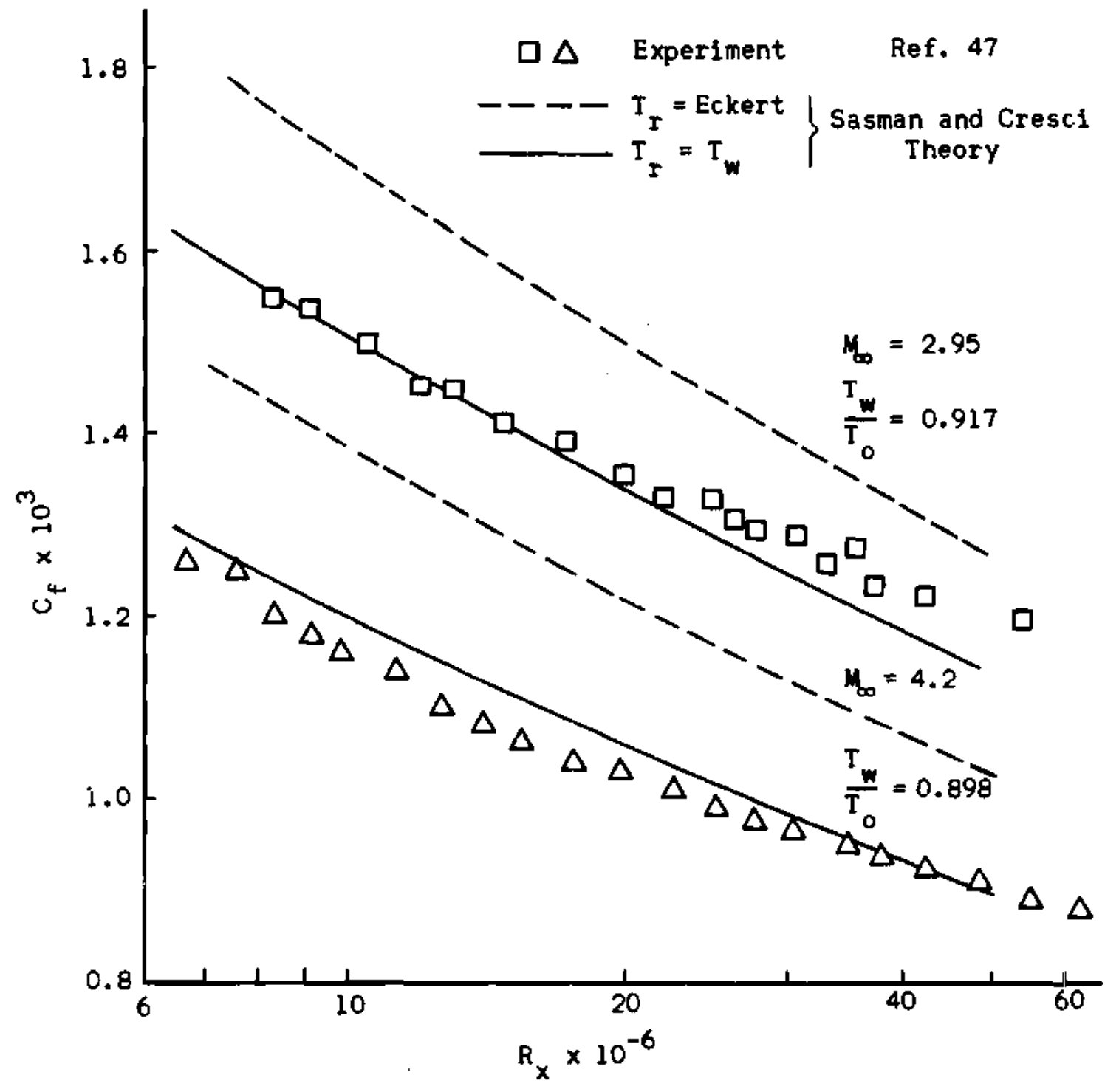

Figure E-1. Insulated Flat Plate Example. 
agreement of experiment and theory is observed when the reference temperature is equal to the wall temperature.

Pasiuk et al. (42) computed the momentum and displacement thicknesses from experimental velocity and temperature profiles for turbulent flow over a flat plate placed in a supersonic nozzle. Data were obtained for both adiabatic and moderately cooled surface conditions. The Mach number distribution for this favorable pressure gradient flow is shown in Figure E-2. The displacement and momentum thickness distributions are shown in Figure E-3 where they are compared with the results of computations using the Sasman and Cresci method. The initial conditions for these solutions were taken from the experimental data at $x=0.6$ and the wall temperatures were taken as constant values representative of the distributions recorded in reference 42. Good agreement is noted between the theory and experiment for both reference temperature assumptions. The $T_{w}$ reference condition gives somewhat better agreement in the zero heat transfer case while there is essentially no basis for preference of either method indicated in the heat transfer example.

The above examples by no means justify the use of the wall temperature as the reference temperature generally. However, they do lend support to its use in the present application of the Sasman and Cresci computation method to the moderately cooled yawed cylinder problem. 


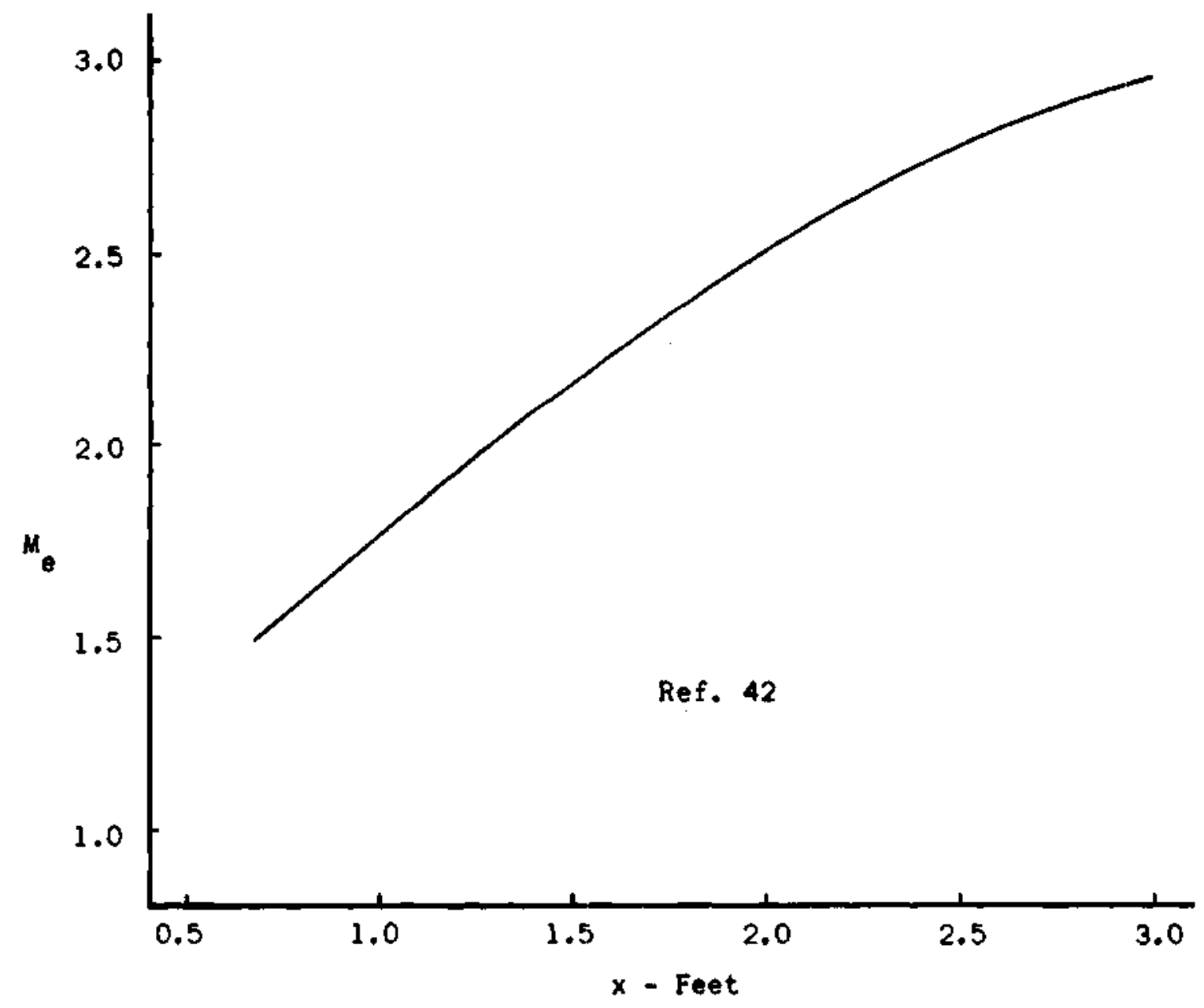

Figure E-2, Mach Number Distribution for Favorable Pressure Gradient Example. 

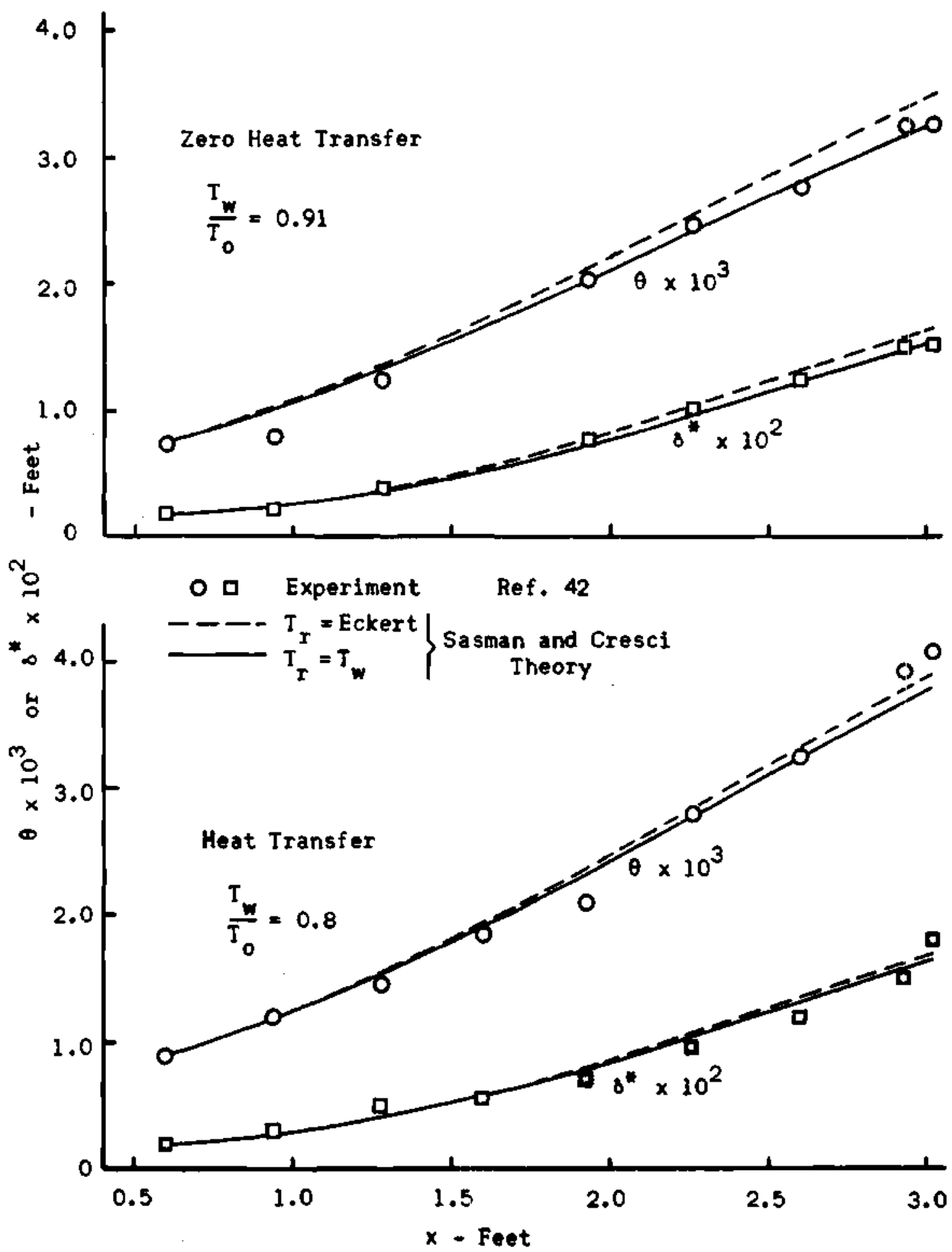

Figure E-3. Favorable Pressure Gradient Example. 


\section{APPENDIX F}

\section{RELATION BETWEEN THE COMPRESSIBLE AND}

TRANSFORMED VELOCITY PROFILES

In the transformed plane the velocity profiles have been assumed to be

$$
\frac{U}{U_{e}}=\left(\frac{Z}{\Delta}\right)^{\frac{H_{i}-1}{2}}
$$

in the streamwise direction and

$$
\frac{V}{U_{e}}=\left(1-\frac{Z}{\Delta}\right)^{2} \frac{u}{U_{e}} \tan \vec{a}
$$

in the cross-flow direction as given by equations 29 and 50 in Chapter III. In the physical plane

$$
\frac{u}{u_{e}}=\frac{u}{u_{e}}
$$

and

$$
\frac{v}{u_{e}}=\left(1-\frac{z}{\Delta}\right)^{2} \frac{u}{u_{e}} \tan a
$$

as a result of the transformation definitions (equations 34,44 , and 47). Specification of the resulting compressible profiles requires a relation between $z / \Delta$ and $\zeta / s$ as given by the Sasman and Cresci transformation 
(equation 24), i.e.

$$
z=\left(\frac{T_{e}}{T_{0}}\right)^{\frac{1}{2}} \int_{0}^{\zeta} \frac{2}{p_{0}} d S
$$

The Crocco relation is written in the form

$$
\frac{T_{s}}{I_{0}}=\frac{T_{w}}{T_{0}}+\left(1-\frac{T_{w}}{T_{0}}\right) \frac{u}{U_{e}}
$$

where $I_{s}$ is the local stagnation temperature. Then noting that $p_{e} / p=$ $T / T_{e}$, for a perfect gas, the density ratio becomes

$$
\frac{\rho_{0}}{\rho}=\frac{p_{0}}{P_{e}} \frac{T_{e}}{T_{0}}\left[\frac{T_{w}}{T_{e}}+\left(\frac{T_{0}}{T_{e}}-\frac{T_{w}}{T_{e}}\right) \frac{u}{u_{e}}-\left(\frac{T_{0}}{T_{e}}-1\right)\left(\frac{u}{u_{e}}\right)^{2}\right]
$$

Now applying the transformation relation

$$
\zeta=\left(\frac{T_{0}}{T_{e}}\right)^{\frac{1}{2}} \int_{0}^{Z} \frac{\rho_{0}}{\rho} d z
$$

to $F .7$ and using F.1 to perform the integration results in the following relation.

$$
\frac{\zeta}{Z}=\frac{P_{0}}{P_{e}}\left(\frac{T_{e}}{T_{0}}\right)^{\frac{1}{2}}\left[\frac{T_{w}}{T_{e}}+\left(\frac{T_{0}}{T_{e}}-\frac{T_{w}}{T_{e}}\right) \frac{2}{H_{i}+1} \frac{U}{U_{e}}-\left(\frac{T_{0}}{T_{e}}-1\right) \frac{1}{H_{i}}\left(\frac{U}{U_{e}}\right)^{2}\right](F, 9)
$$

Finally noting that $\zeta=\delta$ and $Z=\Delta$ at $U / \mathrm{U}_{\mathrm{e}}=1$ 


$$
\frac{\zeta}{\Delta}=\frac{\frac{T_{w}}{T_{e}}+\left(\frac{T_{0}}{T_{e}}-\frac{T_{w}}{T_{e}}\right) \frac{2}{H_{i}+1}\left(\frac{z}{\Delta}\right)^{\frac{H_{i}-1}{2}}-\left(\frac{T_{0}}{T_{e}}-1\right) \frac{1}{H_{i}}\left(\frac{z}{\Delta}\right)^{H_{i}-1}}{\frac{T_{w}}{T_{e}}+\left(\frac{T_{0}}{T_{e}}-\frac{T_{w}}{T_{e}}\right) \frac{2}{H_{i}-1}-\left(\frac{T_{0}}{T_{e}}-1\right) \frac{1}{H_{i}}} .
$$

which relates $\zeta / 8$ to $z / \Delta$. Thus the compressible velocity profiles are determined for a specified inviscid flow when $\mathrm{H}_{i}$ and $\tan \alpha$ are known from the streamwise and cross-flow solutions.

The warping of the velocity profiles as a result of the transformation, as was noted by Reshokto and Tucker (46), is apparent. If for a given station a constant power $\mathrm{N}_{1}$ for the profile of equation $\mathrm{F} .1$ is assumed, then the corresponding physical profile parameter $\mathrm{N}$ of

$$
\frac{u}{u_{e}}=\left(\frac{c}{\delta}\right)^{N}
$$

varies with the normal coordinate $\zeta$. This same type of distortion also exists in the cross-flow profile. It is noted that for the present application at least the distortion is not sufficient to alter the basic parabolic relation shape in the compressible plane. In this sense the application of the parabolic relation in the transformed plane does not conflict with the conclusions from Hall's compressible experiments (24) as quoted in the text. 


\section{APPENDIX G}

\section{MAXIMUM CROSS-FLOW VELOCITY}

The maximum value of the cross-flow velocity $v$ may be simply related to the solution parameters $H_{i}$ and $\tan a$. For the assumed profile of the form (equation 54)

$$
\frac{\mathrm{V}}{\mathrm{U}_{\mathrm{e}}}=\left(1-\frac{\mathrm{Z}}{\Delta}\right)^{\mathrm{n}} \frac{\mathrm{U}}{\mathrm{U}_{\mathrm{e}}} \tan \overline{\mathrm{a}}
$$

the physical velocity may be written

$$
\frac{v}{u_{e}}=\left(1-\frac{z}{\Delta}\right)^{n}\left(\frac{z}{\Delta}\right)^{N_{i}} \tan a
$$

as shown in Appendix $F$ and where $N_{i}=\frac{H_{i}-1}{2}$ and $n$ equal to 1,2 , and 3 corresponds to the linear, parabolic, and cubic profile relations of Appendix C.

Now the maximum value of $v / u_{e}$ is found by first equating the derivative to zero, i.e.

$$
\frac{d\left(v / u_{e}\right)}{d(z / \Delta)}=0
$$

to find the $0<\frac{z}{\Delta}<1$ where $\left(v / u_{e}\right)_{\max }$ occurs. There results

$$
n=1:
$$

$$
\frac{Z}{\Delta}=\frac{H_{i}-1}{H_{i}+1}
$$




$$
\begin{array}{ll}
n=2: & \frac{Z}{\Delta}=\frac{H_{i}-1}{H_{i}+3} \\
n=3: & \frac{Z}{\Delta}=\frac{H_{i}-1}{H_{i}+5}
\end{array}
$$

The corresponding coordinate location in the physical plane may be determined employing equation F.10 of Appendix F. The maximum value of $v$ is then given by equation $G .2$ and the above relations

$n=1$ :

$$
\left(\frac{v}{u_{e}}\right)_{\max }=\left[\left(\frac{H_{i}-1}{H_{i}+1}\right)^{\frac{H_{i}-1}{2}}-\left(\frac{H_{i}-1}{H_{i}+1}\right)^{\frac{H_{i}+1}{2}}\right] \tan a
$$

$n=2$

$$
\left(\frac{v}{u_{e}}\right)_{\max }=\left[\left(\frac{H_{i}-1}{H_{i}+3}\right)^{\frac{H_{i}-1}{2}}-2\left(\frac{H_{i}-1}{H_{i}+3}\right)^{\frac{H_{i}+1}{2}}+\left(\frac{H_{i}-1}{H_{i}+3}\right)^{\frac{H_{i}+3}{2}}\right] \tan a
$$

$n=3$;

$$
\begin{gathered}
\left(\frac{v}{u_{e}}\right)_{\max }=\left[\left(\frac{H_{i}-1}{H_{i}+5}\right)^{\frac{H_{i}-1}{2}}-3\left(\frac{H_{i}-1}{H_{i}+5}\right)^{\frac{H_{i}+1}{2}}+3\left(\frac{H_{i}-1}{H_{i}+5}\right)^{\frac{H_{i}+3}{2}}\right. \\
\left.-\left(\frac{H_{i}-1}{H_{i}+5}\right)^{\frac{H_{i}+5}{2}}\right] \tan a
\end{gathered}
$$

The functional dependence of the maximum value of $v / u_{e}$ on the streamwise incompressible form factor $H_{i}$ is illustrated in Figure $G .1$ for the three assumed profile relations. The rather strong relationship between the streamwise velocity profile and the cross-flow is apparent in the variation of $\left(v / u_{e}\right)_{\max } / \tan a$ with $H_{i}$. 


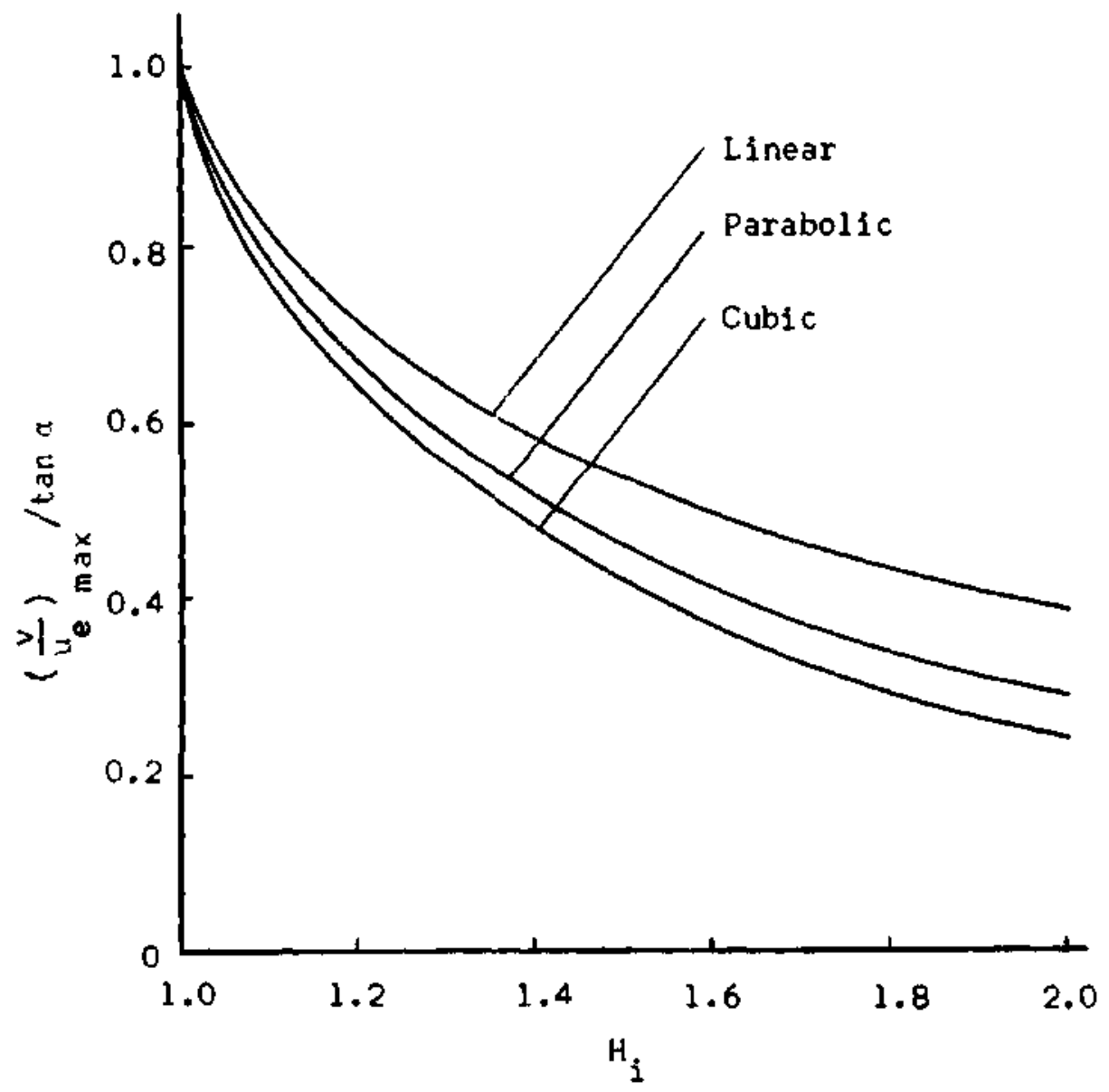

Figure G-1. Effect of $\mathrm{H}_{1}$ on Maximum Cross-Flow Velocity for Three Profile Assumptions. 


\section{REFERENCES}

1. Sears, W. R., "Boundary Layers in Three-Dimensional Flow," Applied Mechanics Reviews, 7, 281 (1954).

2. Moore, F. K., "Three-Dimensional Boundary Layer Theory," Chapter in Advances in Applied Mechanics, Vol.44, Academic Press (1956).

3. Cooke, J. C. and Hall, M. G., "Boundary Layers in Three Dimensions," Chapter in Progress in Aeronautical Sciences, Vol. 2 , Pergamon Press (1962).

4. Cooke, J. C., "Three-Dimensional Turbulent Boundary Layers," ARC C. P. $635(1963)$.

5. Der, J., Jr. and Raetz, G. S., "Solution of General Three-Dimensional Laminar Boundary Layer Problems by an Exact Numerical Method," Paper 62-70 presented at the 30th Annual IAS Meeting, New York (1962).

6. Rosenhead, L. (ed.), Laminar Boundary Layers, Oxford University Press, Gt. Britain (1963).

7. Lindfield, A. W., Pinsent, H. G. and Pinsent, P. A., "Approximate Methods for Calculating Three-Dimensional Boundary Layer Flow on Wings," Chapter in Boundary Layer and Flow Control, Vol. 2, Pergamon Press, London (1961).

8. Eichelbrenner, E. A. and Oudart, A., "Methode de Calcu de la Couche Limite Tridimensionelle, Application a la Corps Fusele Incline Sur le Vent," ONERA Publication No. 76 (1955).

9. Cooke, J. C., "An Axially Symmetric Analogue for Three-Dimensional Boundary Layers," ARC R and M 3200 (1959).

10. Mager, A., "Generalization of Boundary-Layer Momentum Integral Equations to Three-Dimensional Flows Including Those of a Rotating System," NACA Report 1067 (1952).

11. Cooke, J. C., "A Calculation Method for Three-Dimensional Turbulent Boundary Layers," ARC R and M 3199 (1958).

12. Becker, E., "Berechnung Von Reibungschichten Mit Schwacher Sekundärströmung Nach Dem Impulsverfahren," Z. Flugwiss., I, 163 (1959).

13. Zaat, J. A., "Beiträge Zur Thecrie Der Dreidimensionalen Grenzschichten," NLL Report MP 190 (1960). 
14. Gruschwit2, E., "Turbulente Reibungschichten Mit Sekundärstömung," Ing.,-Arch. Bd., 6, 355 (1935).

15. Kuethe, A. M., McKee, P. B., and Curry, W. H., "Measurements in the Boundary Layer of a Yawed Wing," N.ACA TN 1946 (1949).

16. Johnston, J. P., "On the Three-Dimensional Turbulent Boundary Layer Generated by Secondary Flow," J. of Basic Engineering, Series D, Trans. ASME, $\underline{\text { 82 }}, 233(1960)$.

17. Wallace, R. E., "The Experimental Investigation of a Swept-Wing Research Model Boundary Layer," Municipal University of Wichita Aerodynamics Report 092 (1953).

18. Brebner, G. G. and Wyatt, L. A., "Boundary Layer Measurements at Low Speed on Two Wings of $45^{\circ}$ and $55^{\circ}$ Sweep," ARC C. P. 554 (1960).

19. Blackman, D. R. and Joubert, P. N., "The Three-Dimensional Turbulent Boundary Layer," J. Roy. Aeron. Soc., 64, 692 (1960).

20. Braun, W. H., "Turbulent Boundary Layer on a Yawed Cone in a Supersonic Stream," NASA TR R-7 (1959).

21. Vaglio-Laurin, R., "Turbulent Heat Transfer on Blunt Nosed Bodies in Two-Dimensional and General Three-Dimensional Hypersonic Flow," J. Aero. Sci., 27, 27 (1960). Also see WADC TN 58-301 (1958).

22. Beckwith, I. E. and Gallagher, J. J., "Local Heat Transfer and Recovery Temperatures on a Yawed Cylinder at a Mach Number of 4.15 and High Reynolds Numbers," NASA TR R-104 (1961).

23. Spence, D. A., "The Growth of Compressible Turbulent Boundary Layers on Isothermal and Adiabatic Walls," ARC R and M 3191 (1961).

24. Hall, M. G., "Experimental Measurements in a Three-Dimensional Turbulent Boundary Layer in Supersonic Flow," Recent Developments in Boundary Layer Research, Part II, AGARDograph 97, 829 (1965).

25. Mager, A., "Transformation of the Compressible Turbulent Boundary Layer," J. Aero. Sci., 25, 305 (1958).

26. Cooke, J. C., "Boundary Layers Over Infinite Yawed Wings," Aeron. Quart., 11, 333 (1960).

27. Cooke, J. C., "Iurbulent Boundary Layers Over Delta wings at Zero Lift," ARC C. P. 696 (1963).

28. Squire, L. C., "The Three-Dimensional Boundary Layer Equations and Some Power Series Solutions," ARC R and M 3006 (1957). 
29. Beckwith, I. E., "Similarity Solutions for Small Cross-Flows in Laminar Compressible Boundary Layers," NASA TR R-107 (1961).

30. Reshokto, E. and Tucker, M., "Approximate Calculation of the Compressible Turbulent Boundary Layer with Heat Iransfer and Arbitrary Pressure Gradient," NACA TN 4154 (1957).

31. Cohen, N. B., "A Method for Computing Turbulent Heat Transfer in the Presence of a Streamwise Pressure Gradient for Bodies in High-Speed Flow," NASA Memo 1-2-59L (1959).

32. Standen, N. M., "A Concept of Mass Entrainment Applied to Compressible Turbulent Boundary Layers in Adverse Pressure Gradients, "Proceedings of the Fourth Congress of the Internation Council of the Aeronautical Sciences, Spartan Books, Inc., Washington (1965).

33. Sasman, P. K. and Cresci, R. J., Compressible Turbulent Boundary' Layer with Pressure Gradient and Heat Transfer," AIAA J., 4, 19 (1966).

34. Ludwieg, H. and Tillmann, W., "Investigations of the Wall Shearing Stress in Turbulent Boundary Layers," NACA IM 1285 (1950).

35. Eckert, E. R. G., "Engineering Relations for Friction and Heat Transfer to Surfaces in High Velocity Flow," J.Aero. Sci., 22, 585 (1955).

36. Tetervin, N., "The Application of the Reference-Enthalpy Method to Friction Formulas," J. Aero. Sci, 29, 493 (1962).

37. Libby, P. A., Baronti, P. O. and Napolitano, P., "Study of the Incompressible Turbulent Boundary Layer with Pressure Gradient," AIAA J., 2, 445 (1964).

38. Rotta, J. C., "Heat Iransfer and Temperature Distribution in Turbulent Boundary Layers at Supersonic and Hypersonic Speeds, "Recent Developments in Boundary Layer Research, Part I, AGARDograph 97, 35 $(1965)$.

39. Vaglio-Laurin, R., "Laminar Heat Transfer on Three-Dimensional Blunt Bodies in Hypersonic Flow," J. American Rocket Soc., 29, 123 (1959).

40. Ames Research Staff, "Equations, Tables and Charts for Compressible Flow," NACA TR 1135 (1953).

41. Spain, B., Tensor Calculus, Oliver and Boyd, London (1960).

42. Pasiuk, Lo, Hastings, S. M. and Chatham, R., "Reynolds Analogy Factor for a Compressible Turbulent Boundary Layer with a Pressure Gradient," AIAA J., 1,1201 (1963). Also see NOLTR 64-200 (1964). 
43. Cresci, R. J., Mackenzie, D. A., and Libby, P. A., "An Investigation of Laminar, Transitional, and Iurbulent Heat Transfer on Blunt Nosed Bodies in Hypersonic Flow," J. Aero. Sci., 27, 401 (1960).

44. Beckwith, I. E. and Gallagher, J. J., "Heat Transfer and Recovery Temperatures on a Sphere with Laminar, Transitional, and Turbulent Boundary Layers at Mach Numbers of 2.00 and 4.15," NACA TN 4125 (1957).

45. Libby, P. A. and Cresci, R. J., "Evaluation of Several Hypersonic Turbulent Heat Transfer Analyses by Comparison with Experimental Data," WADC TN 57-72 (1957).

46. Reshotko, E. and Tucker, M., "Ef fect of a Discontinuity on Turbulent Boundary-Layer-Thickness Parameters with Application to Shock Induced Separation," NACA TN 3454 (1955).

47. Matting, F. W., Chapman, D. R., Nyholm, J. R., and Thomas, A. G., "Turbulent Skin Friction at High Mach Number and Reynolds Numbers in Air and Helium," NACA TR R-82 (1960). 
VITA

Richard Gordon Bradley, Jr. was born June 18, 1932, in Canton, Georgia. His parents are Richard Gordon and Louise Bishop Bradley. He received his elementary and secondary education in the public school system of Adairsville, Georgia. In 1954 he received the Bachelor of Aeronautical Engineering degree from the Georgia Institute of Technology. After serving for two years in the United States Air Force as an aircraft maintenance officer, Mr. Bradley returned to Georgia Tech where he was awarded the Master of Science in Aeronautical Engineering degree in 1957.

In $1957 \mathrm{Mr}$. Bradley joined General Dynamics, Fort Worth Division, where he worked in applied research in the field of aerodynamics. During the six years he was with General Dynamics Mr. Bradley published a number of corporate reports primarily in the viscous flow area and co-authored one paper published in the open literature dealing with viscous interaction studies.

In September, 1953, he married the former Margaret Lois Boswell of Adairsville, Georgia. They have a daughter, Sandra Kay Bradley, and a son, Richard Gordon Bradley, III. 